\title{
IMPLICATIONS OF THE HUMAN GENOME PROJECT
}

DISTRIBUTION OF THIS DOCUMENT IS UNLITED 


\section{DISCLAIMER}

This report was prepared as an account of work sponsored by an agency of the United States Government. Neither the United States Government nor any agency thereof, nor any of their employees, makes any wartanty, express or implied, or assumes any legal liability or responsibility for the accuracy, completeness, or usefulness of any information, apparatus, product, or process disclosed, or represents that its use would not infringe privately owned rights. Reference herein to any specific commercial product, process, or service by trade name, trademark, manufacturer, or otherwise does not necessarily constitute or imply its endorsement, recommendation, or favoring by the United States Government or any agency thereof. The views and opinions of authors expressed herein do not necessarily state or reflect those of the United States Government or any agency thereof. 


\section{DISCLAIMER}

Portions of this document may be illegible in electronic image products. Images are produced from the best available original document. 


\title{
IMPLICATIONS OF THE HUMAN GENOME PROJECT
}

\author{
Summary \\ Philip Kitcher \\ Department of Philosophy \\ University of California at San Diego
}

1. Introduction

The Human Genome Project (HGP), launched in 1991, aims to map and sequence the human genome by 2006. During the fifteen-year life of the project, it is projected that $\$ 3$ billion in federal funds will be allocated to it. The ultimate aims of spending this money are to analyze the structure of human DNA, to identify all human genes, to recognize the functions of those genes, and to prepare for the biology and medicine of the twenty-first century.

The following summary examines some of the implications of the program, concentrating on its scientific import and on the ethical and social problems that it raises. Its aim is to expose principles that might be used in applying the information which the HGP will generate. There is no attempt here to translate the principles into detailed proposals for legislation. Arguments and discussion can be found in the full report, but, like this summary, that report does not contain any legislative proposals.

\section{The Scientific Impact of the HGP}

The mapping of the human genome is well under way, and the production of fine-grained genetic and physical maps has already borne fruit in the accelerated pace with which genes associated with human diseases are being identified. There is little doubt that the mapping goals of the HGP will be achieved by 2006 . 
The project to sequence the human genome is intended to prepare the way for an enormous number of future projects in biology and medicine. From information about sequence, using comparisons with known gene sequences in human beings, and, most importantly, in other organisms, it should be possible to identify a very large proportion of the human genes, to understand their products, and ultimately, the role that those products play in human physiology. The task of moving from sequence data to biological understanding is extraordinarily complex, and it can only be undertaken by way of a thorough understanding of nonhuman organisms, on which detailed experimental manipulations are possible. It is thus essential to sequence the genomes of yeast, the nematode worm $C$. elegans, the fruit fly Drosophila melanogaster, and (if possible) the mouse. These "model organisms" are not incidental to the project. They are at its center.

Sequencing rates must be improved by a factor of about 100 if the goal of sequencing the human genome is to be achieved within the next decade. There is no doubt that the genomes of yeast, the nematode, and the fruit fly will be sequenced. If it does not prove possible to increase rates of sequencing by the amount needed, then indirect strategies of identifying human genes can be employed. These will probably not produce as complete a catalog of the human genes, but they will deliver a very large amount of biologically significant information. The knowledge that we shall achieve, on either strategy, will be primarily concerned with features that we share with many other organisms, but this knowledge will greatly illuminate our understanding of human disease. It is already abundantly clear, that, whether or not the official goal of delivering the full human sequence is reached, the information generated by the HGP will have extraordinary value. 


\section{Genetic Testing}

Genetic testing appeared before the HGP began and would continue even if the HGP had not been funded. However, the HGP will accelerate the pace at which genetic tests become available. Tests may be useful in a variety of contexts: in diagnosing disease, differentiating cases of a known disease, predicting risks for disease, identifying genetic conditions in a fetus, and discovering if both members of a couple carry recessive alleles. The diagnostic uses are valuable, and relatively uncomplicated.

If predictive tests are to provide meaningful information, it is crucial that there should be background statistics on the frequency with which bearers of the pertinent combination of genes become afflicted with the disease, preferably statistics that also assess variation with respect to environment. Some tests that have already been developed are potentially highly misleading because of ignorance about the prevalence of the genes in the asymptomatic population.

Many existing examples (Huntington's Disease, Tay-Sachs Disease, sickle-cell anemia and others) show that we may well have the ability to test long before we have the skill to treat or prevent a disease. It is crucial that the medical and nonmedical benefits provided by genetic tests should be evaluated, preferably by a body that has no commercial interests in the distribution of the tests, and that this information should be clearly communicated to doctors and their patients. There is currently a danger that tests without value will be automatically recommended. Moreover, an increase in the number of genetic tests should be accompanied by expansion of genetic counselling. It is already obvious that more counsellors will be needed, and that they will have to be trained in ways that enable people to make difficult decisions, including the decision about whether they should take a genetic test.

.. There are occasional circumstances under which the mandatory use of genetic tests can be recommended (as, for example, in the case of PKU).. In the 
overwhelming majority of instances, however, patients can and should make their own choices. At the present stage it is far from obvious that there are many diseases for which population-wide testing would be appropriate.

\section{Genetic Discrimination}

Genetic information about individuals can be used to their detriment, and steps should be taken immediately to protect people from genetic discrimination.

Discrimination is primarily a threat in relation to insurance and to employment, although social stigmatization may also occur, either independently of or in conjunction with familiar forms of prejudice.

In the past, some kinds of genetic discrimination have been caused by misunderstandings of the scientific results. Those who wish to employ genetic information should clearly understand that they are responsible for applying the science in an accurate fashion.

Aleady, a significant number of people have been denied health insurance, or offered health insurance only at very high premiums, because they carry a genetic condition. Elementary considerations of justice lead to the conclusion that genetic information should not be used in this way, since people cannot justly be victimized for circumstances that are beyond their control. The introduction of genetic tests reinforces the argument for universal health care coverage.

Genetic information can also be used in setting premiums for life and disability insurance. While the argument in these cases is not quite as compelling as for health insurance, basic principles of justice again support the conclusion that insurers should not be able to use genetic information in setting premiums.

Employers may wish to use genetic tests for a number of reasons: they may wish to avoid high insurance premiums, or losses in worker productivity, or expensive modifications of the workplace environment. If the recommended solutions to the 
problems of health and disability insurance are adopted, then the first type of motivation is automatically removed.

Remaining instances in which employers wish to use genetic tests, in making decisions either about workers or about applicants, may involve conflicts of interest between the employer and those tested. These conflicts should be resolved by courses of action that accord with the following principles.

1. Employers typically have obligations to employees that they do not have towards applicants, obligations that are greater, other things being equal, when the period of employment has been longer. These obligations make a difference to the uses that employers can make of genetic tests: more stringent requirements must be met in using a genetic test to deny an employee a coveted position than in using a genetic test to refuse a position to an applicant.

2. It is justifiable to use genetic tests to debar people from positions when those tests identify genetic conditions which would cause their bearers, in the environments associated with the position, to be at appreciably higher risk for acting in ways that would endanger the health of others.

3. Genetic testing for general risks can occasionally be justified in assessing the qualifications of applicants. Employers have the right to take into account the expected length of an applicant's career, especially in circumstances that demand a heavy initial investment in training. But the risks must always be considered in the context both of the normal turnover of employees, and the candidate's other qualifications.

4. Genetic tests should not be used to determine the general health and disability risks of those already employed. 
5. Employers have the duty to modify the workplace environment, when they can do so in an economically feasible way. Applicants have no right to protest genetic testing when that testing is for workplace specific risks, and when they have equally good employment opportunities in environments that do not pose a threat to them. When the environment is unmodifiable, conflicts between employers and applicants over the use of genetic tests can - at least sometimes - be settled through retraining programs and job creation programs. Employers who benefit from the absence of competition for their employees, and who use genetic tests to distinguish among applicants, have the responsibility to contribute significantly to the costs of such programs.

Where the differences in risk among the bearers of alternative genotypes depend on the amount of some substance released in the workplace environment, then any decision must proceed on the basis of a clear understanding of this dependence and on how it relates to the economic constraints on modifying the environment.

There is a real danger that genetic information will reinforce existing prejudice against the members of particular groups. This has come about in the past partly as the result of ignorance, partly because of entrenched social attitudes. Offices that attempt to promote equal opportunity should be thoroughly informed, both about the general possibility of cloaking prejudice in scientific claims about genotypes, and about the particular kinds of correlations that molecular genetics has discovered.

\section{Prospects for Therapy}

The ultimate goal of the HGP is to treat and/or prevent major diseases. Genetic tests will be the first fruits of the project, and, as noted, tests will sometimes, perhaps often, be available long in advance of possibilities of therapy. Nevertheless, there are reasons to think that increased biological understanding will lead to novel 
forms of therapy for hereditary diseases, and, possibly, for other kinds of disease as well.

There are many distinct ways in which molecular biology can contribute to the treatment of disease: by showing us what protein products are missing and need to be delivered, by revealing that particular molecules need to be broken down, by uncovering ways to identify and incapacitate cells that malfunction. The emphasis of the HGP on finding a very large proportion of the human genes will probably lead us to systematic ways of treating what now appear as very different kinds of disease.

It is quite wrong to think that the principal form of therapy to emerge from molecular genetics will involve replacement of genes, but insertion of DNA into cells in a patient's body is one strategy that future molecular medicine will employ. At present, gene replacement therapy is limited by our ability to target the intended recipient cells and to control the expression of the genes delivered to them. A particularly severe problem is the delivery of genetic material to neurons. Currently, gene replacement therapy is primarily directed at extremely debilitating genetic diseases for which niceties of timing are irrelevant.

Gene replacement raises ethical problems, but we should avoid the simplistic slogans that debar gene replacement either in germline cells or for purposes of trait enhancement. The crucial issues concern the risks of the procedure, the need to preserve the identity of the patient, and the consent of those affected. At present, the risks of gene replacement are sufficiently great in some cases to render unjustifiable gene replacements in either somatic or germline cells. If our techniques become much more precise, then gene replacement may be safe both with respect to the soma and in the germline. Some forms of gene replacement may also prove necessary to preserve children from inevitable neural degeneration, and, in such cases, concern for their welfare may override the absence of their consent. Finally, if it becomes possible to develop forms of gene therapy that enhance our resistance to infectious disease, then it 
would be wrong not to carry out the genetic replacement simply to pay lip service to the slogan that we must only use gene therapy to cure diseases.

For the foreseeable future, decisions about gene replacement should be undertaken on a case-by-case basis with due attention to the morally significant factors: the risks, the preservation of the patient's identity, and the patient's consent.

\section{Forensic Uses of DNA Technology}

Information about DNA sequences of individuals can be used in a number of identification projects: to reunite families, to identify corpses, to check putative relationships, and to investigate and prosecute crimes. The last is the most common.

Forensic uses of DNA currently proceed by comparing the bodily materials (blood, semen, hair) left at the scene of a crime with a sample (blood, a cheek swab) obtained from a suspect. The crime scene sample cannot be analyzed completely. Instead, a DNA profile is constructed by analyzing selected regions that are variable from person to person. If the DNA profile from the crime scene sample fails to match that of the suspect then the suspect can be excluded.

Controversies about the use of forensic DNA stem from the possibility that matching profiles may occur fortuitously. To obtain convincing evidence against a suspect it is necessary to show both that the profiles match, and that it would be very improbable for the DNA profile from anyone else to match the crime scene DNA. In the past, this has been done by analyzing a number of different regions that are assumed to assort independently. However, there are serious concerns that the regions chosen may not be independent, and that the probabilities announced by prosecutors are misleading. Settling these issues requires detailed statistical sampling of appropriate populations, and it seems clear that the kinds of probabilities typically claimed are very hard to support thoroughly. In any case, there is little point in showing that the 
probability of a fortuitous match is exceptionally small, when it has to be acknowledged that the chance of a laboratory error is significantly greater.

Technical controversies in statistics and population genetics could, in principle, be bypassed if there were a complete national DNA database. There are two main worries about a database of this kind. One is that it would constitute an invasion of privacy; the other is that it would be costly. If the regions of the genome used in constructing DNA profiles are appropriately chosen, then DNA profiles can be reduced to a set of meaningless numbers, and, by enforcing procedures for the protection of individuals (as detailed in the full report), it would be possible to employ a DNA database without invasions of privacy. For the present, complete population typing is likely to prove expensive, but it must also be recognized that the collection of a sufficient body of statistics to support conclusions about the chance of fortuitous matching of DNA profiles is also costly. A national DNA database would have the added advantage of serving as an instrument of detection as well as one of prosecution.

\section{Long-Range Implications}

Many critics of the HGP are concerned that it will contribute to a revival of the unsubstantiated claims about genetic determination of behavior that have sometimes threatened to affect public policy. It is extremely important to promote public understanding of the fact that genes and environments cause the traits that interest us about ourselves. It is also crucial to recognize the difficulty of the problems that the genetics of human behavior faces, and the limitations of the methods that this discipline has at its disposal. Although the HGP is likely to transform our understanding of human genetics, the principal advances will almost certainly be in those areas in which we can test our conclusions on nonhuman organisms that exhibit traits similar to ours.

Another widespread fear is that the HGP will contribute to a new kind of eugenics. Given the evils of eugenic practices, both in Nazi Germany and in the USA 
during the 1920s and 1930s, this concern is readily understandable. However, if the HGP will contribute to a "eugenic" program, it will be very different from the historical programs that rightly arouse anger and disgust. One significant difference lies in the fact that our use of the new information from molecular genetics will give individuals the opportunity to make their own reproductive decisions, instead of imposing some central policy about what kinds of people there should be.

The overriding of individual values is probably the most disturbing feature of the eugenics of the past. However, it is surely too simple to believe that simply allowing a free market in reproductive technologies will empower prospective parents to make the decisions that reflect their own ideals. Without a broad respect for difference, and a shared determination to enable those who are born to develop their potential, individual reproductive decisions will be affected by social preferences for certain types of people. If molecular genetics becomes commonly applied in pre-natal contexts, then it will be necessary to couple the provision of tests with a wellrecognized policy of providing extensive services for those who are born with disabilities.

Although the topic of abortion is not common in the rhetoric around the HGP, there is no denying the fact that the new molecular genetics is likely to increase the number of abortions. Proponents of the HGP are tacitly committed to the principle that a society in which fewer children are born with genetic disabilities, because of prenatal testing and selective abortion, is better than one with a higher rate of birth defects. There are serious concerns about this principle, which need public discussion. The full report outlines some of the major issues.

Finally, the success of the HGP will raise important questions about priorities. It will offer us the option of replacing reactive medicine with preventative medicine. To the extent that we invest in prevention, then we shall have to decrease our expenditures on terminal care, unless we add further to the costs of medicine. 
Eventually, the HGP may bequeath to us a medical practice dedicated, not to curing disease and prolonging life, but to enhancing the opportunities of the young, preventing the onset of diseases that limit activity, and to ensuring the possibility of a quick and painless death for those whose quality of life has become severely reduced.

Finally, it is important to remember that the HGP is part of an international project. As greater understanding of the molecular basis of disease becomes available, researchers in affluent countries may face even broader questions of setting priorities. We shall have to ask ourselves whether we should concentrate on our local killers (such as cancer) or on diseases (like malaria) which cause the death of millions of children each year. A coherent disease policy will ultimately have to be integrated with a serious population policy. As the HGP fulfils its promise it will surely confront our successors with questions of extraordinary difficulty. But we must remember that different versions of those questions would face us even in the absence of molecular understanding, and that ignorance is unlikely to prepare us better to meet our challenges. 


\title{
IMPLICATIONS OF THE HUMAN GENOME PROJECT
}

\author{
Philip Kitcher \\ Department of Philosophy \\ University of California at San Diego
}

La Jolla, CA. 92093 


\section{ACKNOWLEDGEMENTS}

I would like to thank the many people who have enlightened me about various issues. Conversations with George Annas, David Botstein, Mario Capecchi, L.L. Cavalli-Sforza, Patricia Churchland, Bob Cook-Deegan, Norman Daniels, Larry Deaven, Russell Doolittle, Dan Drell, Troy Duster, Glen Evans, Ted Friedmann, David Galas, Walter Gilbert, Steve Gould, Stephen Hilgartner, Leroy Hood, Ruth Hubbard, Marilyn Jones, Eric Juengst, Michael Kaback, Stuart Kaufman, Evelyn Fox Keller, Dan Kevles, Eric Lander, Michael Levine, Richard Lewontin, Robert Moyzis, Dorothy Nelkin, Marvin Notowicz, Maynard Olson, Diane Paul, Hilary Putnam, Philip Reilly, Jasper Rine, Gerald Rubin, Barbara Katz Rothman, Mike Rothschild, Tim Scanlon, Ken Schaffner, John Sulston, Tim Tully, Nancy Wexler, Ray White, Chris Wills, Jan Witkowski, Jim Woodward, and Michael Yesley have been valuable and instructive. Anthony Carrano and his colleagues at the Lawrence Livermore Genome Center, and Ray Gesteland deserve special thanks for the extensive help and warm hospitality they provided. I have also benefitted from discussions with Kenna Barrett, Linda Derksen, Joan Esnayra, Ilya Farber, Bruce Glymour, Mara Harrell, Josh Jorgensen, Brian Keeley, Alex Levine, Kerry Monda, Michael Selgelid, and Scott Sterling. I received helpful comments on an earlier draft from Kenna Barrett, Bob Cook-Deegan, Joan Esnayra, Prosser Gifford, Patricia Kitcher, Alex Levine, Mike Rothschild, Ken Schaffner and Michael Selgelid. I am grateful to Josh Jorgensen and Alex Levine who produced the figures for me. Particular thanks are due to Sylvia Culp and Dorothy Nelkin, whose suggestions were particularly insightful. Finally, I owe an immense debt to Bill Loomis, for numerous conversations and exceptionally detailed comments on various drafts. Without his advice, this report would contain a far higher number of errors and misjudgments.

I am extremely grateful to Declan Murphy for inviting me to undertake this project, to Claudette Friedman for her tremendous understanding and efficiency, and to Prosser Gifford for his interest and support. 


\section{REPORT ON THE HUMAN GENOME PROJECT}

\section{Contents}

1. Introduction

2. Some Molecular Biology

$\begin{array}{llr}2.1 & \text { Classical Genetics } & 4 \\ 2.2 & \text { DNA, RNA, and Proteins } & 7 \\ 2.3 & \text { Cloning } & 10 \\ 2.4 & \text { Mapping } & 13 \\ 2.5 & \text { Sequencing } & 15 \\ 2.6 & \text { The State of the Art } & 17 \\ & \text { Figures for Section 2 } & 20\end{array}$

3. The HGP and Beyond

3.1 Conflicting Claims about the HGP 32

3.2 Sequence and Beyond: The Role of Model Organisms 35

3.3 Can it be done? 42

4. The Potential and Pitfalls of Genetic Testing

4.1 Introduction $\quad 47$

4.2 Contexts of Genetic Testing 48

4.3 Examples of Conditions Subject to Genetic Test 50

4.4 Reliable Information? 56

4.5 Nothing to be Done? 64

4.6 Problems of Communication? 70

4.7 Forbidding, Requiring, and Encouraging Genetic Tests 73

4.8 Population-Wide Testing 78

4.9 Summary of Main Conclusions $\quad 82$

$\begin{array}{ll}\text { Technical Note } 1 & 84\end{array}$

Technical Note 2

5. The Threat of Genetic Discrimination

5.1 Two Sources of the Value of Privacy 90

5.2 Contexts of Genetic Discrimination 93

5.3 Misinformation and Injustice 95

$5.4 \quad$ Health Insurance $\quad 97$

$\begin{array}{lr}5.5 \text { Life and Disability Insurance } & 104\end{array}$

5.6 Discrimination in the Workplace 107

$\begin{array}{ll}5.7 & \text { Social Stigmatization } \\ & 120\end{array}$ 
5.8 Reinforcing Prejudice 124

Figure 5.1

6. Prospects for Therapy

6.1 Multi-Levelled Possibilities 128

6.2 Gene Replacement -- Necessity and Possibility 132

6.3 Ethical Questions of Gene Replacement 136

7. Forensic Uses of DNA Technology

7.1 Identifying People by their DNA 152

7.2 Failures of Independence 155

7.3 Identifying the Population 159

$\begin{array}{ll}7.4 \text { Laboratory Error } & 161\end{array}$

7.5 Population-Wide Typing 163

7.6 The Scope of Forensic Uses of DNA

$\begin{array}{lll}7.7 & \text { Options } & 174\end{array}$

8. An Overview of Some Further Issues

8.1 Introduction 177

8.2 Genetic Determinism 177

8.3 Eugenics 186

8.4 Abortion and Genetic Testing 194

8.5 Priorities 205

$\begin{array}{ll}\text { References } & 209\end{array}$ 


\section{Introduction}

The Human Genome Project (HGP), launched in 1991, aims to map and sequence the human genome by 2006 . During the fifteen-year life of the project, it is projected that $\$ 3$ billion in federal funds will be allocated to it. The ultimate aims of spending this money are to analyze the structure of human DNA, to identify all human genes, to recognize the functions of those genes, and to prepare for the biology and medicine of the twenty-first century (DOE/NIH 1990; Caskey 1992).

Even at the early stages of planning the HGP, it was evident that the genetic information it would generate would have social impact, and $3 \%$ of the budget was designated for the exploration of the ethical, legal, and social implications (unfortunately known as "ELSI" issues). Much of the report that follows is focused on the ethical and social implications of the project. However, it has also seemed worth scrutinizing more closely the kinds of scientific information that the HGP is currently generating, and those that it may be expected to yield in the near future.

Section 2 offers a brief, non-technical, account of pertinent parts of molecular biology, introducing vocabulary that will be used in later discussions. Section 3 considers the utility of the information generated by the HGP, and the feasibility of completing the project. The discussion of section 3 is a necessary preliminary for assessing the long-range impact of the HGP.

Consideration of ELSI issues begins in section 4. Because genetic tests will be the first fruits of the project, the first questions to be addressed concern the value of such tests. What kinds of information will be most useful for individual decisionmaking? What background resources will be required if tests are to enhance people's abilities to make autonomous decisions? When, if ever, should a genetic test be recommended for all members of the population? Are there some genetic tests that should be forbidden? 
When genetic information about individuals is generated, there are legitimate concerns about the invasion of privacy. These concerns have often been reflected in demands for a genetic privacy act. Section 5 begins by considering the reasons why people might wish to keep information about themselves (including genetic information) private, arguing that the main source of concern is not the release of the information itself but the uses that might be made of that information. It continues by distinguishing a number of contexts in which genetic information about a person might be used to harm that person: in decisions about medical insurance, life insurance, disability insurance, in employment decisions, and in the more nebulous matrix of social attitudes. The main enterprise of section 5 is to consider when genetic information might legitimately be used in these contexts, and when uses of such information would be discriminatory. The section concludes with some guidelines for policies that might be introduced to combat the threat of genetic discrimination.

Even if genetic tests are likely to be the first fruits of the HGP, the hope is, of course, that the information acquired will lead to methods of intervening to prevent, control, treat, or cure diseases. Section 6 reviews the ways in which the knowledge gained might be applied in medical interventions, stressing the advantages that may accrue from global understanding of the human genome. It then takes up the vexed issue of gene replacement therapy and its moral status.

Section 7 considers the uses of DNA evidence in forensic contexts. After reviewing problems with some courtroom claims about DNA evidence, ways of solving these problems are considered. The section concludes with discussion of the idea of DNA typing, either for a whole population, or for special subpopulations, examining the social and economic costs as well as the potential benefits in resisting crime.

The questions taken up in Sections 4-7 are, in the main, issues that will confront us in the near future. But the HGP also has implications on a longer time-scale. Some people are enthralled, others appalled, by the idea that molecular genetics will be 
applied to study human behavior. Because the HGP is likely to make a wide range of fetal tests possible, commentators have voiced concerns about a "new eugenics" (Duster 1990). Section 8 takes up these questions, in a brief and very preliminary fashion. They are not so obviously urgent as concerns about forensic evidence or genetic discrimination, but they may have an even more profound impact in the long-term. Public discussion of them should surely begin now.

This report ignores some important problems, and downplays some aspects of the problems that it treats. Nothing is said about the currently contested question of patenting DNA sequences. Patenting issues cannot readily be detached from the legal context, and, throughout, the report does not delve into legal details. Its aim is to expose arguments and principles that might be used to reach decisions on major ELSI questions. Translating these arguments and principles into appropriate legislation lies beyond the author's competence. 


\section{Some molecular biology}

\section{$2.1 \quad$ Classical Genetics}

Most people are familiar with some simple cases of hereditary transmission. Brown-eyed parents can have blue-eyed children without suspicions of infidelity: blue eyes only occur in people who have two copies of a hereditary factor - two copies of the "blue-eye gene"; people with one "blue-eye gene" and one "brown-eye gene" have brown eyes; so two parents with brown eyes may both have one copy of the "blue-eye gene", and if both pass on their "blue-eye gene" to a child, then the child will have blue eyes. Fundamental to this story is the idea that the factors passed across the generations come in pairs, one transmitted by each of the parents, and that it is the pairwise combinations that affect the observable traits people have. So-called dominant genes show their effects whenever they are present; recessive genes reveal themselves only when they occur in double dose. The gene for blue eyes is recessive.

Genes play two roles. They are transmitted from parents to offspring, and they help to cause the observable traits (the phenotype) of those who bear them. "Help to cause" is important here: it is fundamental to recognize, from the beginning, that talking about the effect of an individual gene can be seriously misleading. Genes act together, so that the phenotype is a function of the totality of genes (the genotype). But it is not a function of the genotype alone. Together, the genotype, the internal composition of the fertilized egg (the zygote) and the environment in which the organism develops, determine the phenotype. Every gardener should understand the point. No matter how high the quality of the genes, the nature of the environment can have profound effects on whether or not the plants thrive.

Since the first decade of the twentieth century, it has been recognized that genes are segments of chromosomes, structures within the cell which are readily recognized under the microscope. Chromosomes come in pairs in sexually reproducing 
organisms, and when the sex cells (the gametes, the sperm and eggs) are formed, they receive one chromosome from each pair. (This is meiotic division). Human beings have 23 pairs of chromosomes, so a human cell which is not a gamete (that is, a somatic cell) contains 46 chromosomes, while gametes only contain 23 . When the sperm and egg unite to form a zygote, the typical number of chromosomes -- 46 -- is restored. Genes occupy distinctive positions, loci, on chromosomes. Thus, for example, the blue-eye gene and the brown-eye gene are alternative forms of the genetic material at the same locus: they are said to be alleles of the same gene. If an organism has two alleles of the same kind at a locus, then the organism is said to be homozygous at that locus (it is a homozygote); if it has different alleles at the locus, then it is heterozygous at that locus (a heterozygote).

Imagine that you are heterozygous for two loci on the same chromosome pair (geneticists typically say "same chromosome", but we are really talking about different alleles at the same locus on a pair of chromosomes), as shown in Figure 2.1. At the first locus on one chromosome you have $A$, and at the second locus on that chromosome you have $B$ (the letters are names for alleles); on the corresponding chromosome, you have $a$ and $b$ respectively. Now, given the story so far, it seems that there are just two choices: you will give your offspring either the combination $A B$ or the combination $a b$, because the loci are linked together. However, matters are not so simple. Before the meiotic division in which your gametes are formed, the chromosomes pair up and exchange genetic material. Linkage of loci is not absolute, and there is a chance that the connection between the loci may be cut, with exchange of genetic material. The further apart two loci on the same chromosome are, the more places there are between them, and thus the higher the chance that there will be genetic recombination between them. Geneticists are able to use this fact to determine the order of the loci (or the genes) on a chromosome. If the $B$-locus lies between the $A$ - 
locus and the $C$-locus, then there will be recombination between $A$ and $C$ more frequently than between $A$ and $B$ (or than between $B$ and $C$; see Figure 2.2). This strategy has been used since the early decades of the century to produce genetic maps of organisms, diagrams that show the order of the loci along an organism's chromosomes. When an organism can easily be manipulated in the laboratory, by "marking" it with various genes (making sure that its genome contains combinations of alleles that, in the environments created in the experiment, cause the organism to have some readily detectable feature) and choosing matings cleverly, it is possible to develop quite detailed genetic maps, as geneticists have done for the fruit fly Drosophila melanogaster. Until recently genetic mapping in human beings had to be content with very little detail.

Classical genetic analysis of our species is handicapped by our inability to use the geneticists' standard approaches on ourselves: it would be profoundly unethical to control human matings as we manipulate fruitflies. Human geneticists must take cases of genetic variation as they find them, and do their best to understand the genetic factors responsible for the patterns they see. It is crucial to recognize, from the beginning, that phenotypic traits are the joint products of hereditary factors -- genes -and environmental factors (which are, in many instances, of such complexity that we do not even know how to classify them). Until we can forge a link between our own genes and those of other organisms, we cannot take the most direct steps towards understanding the ways in which genotypes and environments produce phenotypes. The promise of molecular biology in general, and of the HGP in particular, is that it will provide ways of making the connection. 


\subsection{DNA, RNA, and Proteins}

Chromosomes have two kinds of constituents: proteins and DNA. Before the 1940s, most biologists assumed that the genetic material resided in the protein portion, but experimental work revealed that genes are made of DNA. In 1953, James Watson and Francis Crick identified the molecular structure of DNA (see Figure 2.3). Because the two strands of a DNA molecule are complementary -- Adenine (A) always pairs with Thymine $(T)$, Cytosine $(C)$ with Guanine $(G)$-- there is a straightforward explanation of how DNA is replicated in the mitotic divisions through which somatic cells give rise to their descendants (as well as in the meiotic divisions): the two strands of the helix separate, each serving as the template for a complementary strand.

DNA molecules differ in the exact sequences of the bases (nucleotides), $\mathrm{A}, \mathrm{C}$, G, T. How do these differences in the genetic material affect the phenotypes of organisms? Geneticists began to answer this large question, by studying the mechanisms of immediate gene action. When a gene becomes active in a cell (when it is switched on, or expressed), a complementary strand of RNA is formed. (RNA, ribonucleic acid, is similar to DNA; two important differences are that it is typically single-stranded, and that the base Thymine (T) is replaced by Uracil (U)). This RNA, messenger RNA or $m R N A$, moves to a site in the cell at which proteins are formed. Proteins are composed of amino acids linked by peptide bonds, and the order of the amino acids is determined by the sequence of bases in the mRNA. DNA is transcribed into mRNAs; mRNA is translated into proteins.

We can now begin to understand genetic differences and their effects. If we alter the DNA sequence in a functioning gene, we shall affect the sequence of bases in the corresponding mRNA, and this may make a difference to the protein that is formed. Any such difference may affect one or more chemical reactions that occur as the 
organism grows, develops and interacts with its environment. Depending on the causal details the impact on the phenotype may be slight - even so minute as to escape notice -- or omnipresent. But, in every case, that impact will come about as the result of the ways in which proteins interact with one another and with molecules in the organism's surroundings.

The relation between RNA sequences (ultimately DNA sequences) and the proteins that are formed has been uncovered: in the 1960s, biologists cracked the "genetic code". Bases along an RNA molecule are read in threes, each triplet coding for a particular amino acid or signalling the termination of the growing protein chain. We can now understand why some changes in a DNA molecule might be more crucial than others. If a mutation occurs that simply switches one base within a gene, all that will happen is that the corresponding protein, which may have hundreds of amino acids strung together, will have a different amino acid in (at most) one place. (The consequences of this should not be underestimated; the disease sickle-cell anemia results from just such a single amino-acid substitution.) Insertions or deletions within the genetic material are potentially more catastrophic, for these can alter the reading frame (determined by the exact base at which transcription starts; see Figure 2.4), changing a large number of amino acids, and usually inserting a premature stop codon (a triplet of bases that codes for ending transcription), thus causing a truncated protein. Twenty amino acids are found in living organisms, and elementary arithmetic shows that there are 64 codons (triplet sequences; because there are four possibilities -- A, C, G, T -- at each of three places, the number of combinations is $4^{3}$ ). So the genetic code is redundant (or degenerate): six codons code for the amino acid leucine, four codons code for valine, and there are three stop codons.

In multicellular organisms (like ourselves) the genetic material is virtually the same in most somatic cells; sometimes there are slight modifications of the DNA in 
mitotic divisions; some cells -- lymphocytes and erythrocytes have special genetic features. But cells divide into different types, muscle cells, neurons, skin cells, and so on, all with distinctive properties. Even if all the genes are the same, different cells must activate some genes in different ways. To understand the causes of our phenotypic traits, we will have to consider the problem of gene regulation, the selective switching on and switching off of genes. Molecular biologists know the fine details for only a few genes in a few organisms (notably the intestinal bacterium $E$. coli), but the shape of the general solution is clear. Gene expression is controlled by pieces of DNA sequence outside (and, sometimes, within) a gene, and interactions between these sequences and molecules in the cell determine whether or not the gene is accessible to those molecules that initiate transcription. Two points deserve emphasis. The processes of transcription and translation should not be viewed as simply bits of DNA making bits of RNA or bits of RNA making protein: a whole army of helper molecules is involved; which helpers are available will depend on the protein composition of a cell, so that cells containing different proteins can be expected to express different genes. Second, in many organisms, including the more complex multicellular organisms, the density of genes along the chromosomes is rather low. Between the genes, the sequences of DNA that code for RNAs (and, ultimately, for proteins) there are noncoding regions. The parts of the noncoding region relatively close to a gene may play a role in the expression of that gene, but many geneticists believe that large parts of our DNA sequence have no function, that they are simply vestiges of our evolutionary history. We can think of our genome as a peculiar volume in which an author, addicted to revisions, has bound all the early drafts along with the final version.

There are significant differences between the most simple organisms, the prokaryotes whose cells lack a nucleus, and the eukaryotes which contain their chromosomes within a nucleus. In the prokaryote $E$. coli, the process from DNA to 
RNA to protein usually goes in an uncomplicated way: the sequence of bases in a gene (a coding region of DNA) corresponds exactly with the sequence on the mRNA and that corresponds exactly (by way of the genetic code) to the sequence of amino acids in the protein. Eukaryotes go in for mysterious complications. After transcription, the RNA is modified, and an important feature of the modification is that certain pieces of sequence are snipped out. Only selected parts of the continuous stretch of DNA that gave rise to the original RNA are encoded in the amino acid sequence finally made: these parts are the exons; the intervening sequences (or introns) correspond to those parts of the original RNA that are removed before translation. (See Figure 2.5).

\subsection{Cloning}

Typical cells contain many molecules for chopping up and joining together pieces of DNA and RNA. One of the great discoveries of the 1970s and 1980s consisted in finding ways to put these molecules - restriction enzymes --to work for human purposes. Restriction enzymes recognize particular nucleotide sequences and cut the DNA at sites within (or nearby) those sequences (see Figure 2.6). Whole chromosomes can be digested with restriction enzymes, which break them up into a collection of specific fragments. If the fragments are of the right size, they can be combined with the DNA of other organisms (using ligases), and these organisms can be tricked into making many copies of the recombinant DNA (the organism's own DNA plus the inserted foreign DNA). Restriction enzymes can be thought of as molecular scissors, ligases as molecular paste, and the organisms that do the work (the hosts) as molecular copying machines.

The purpose of cloning is to make large numbers of copies of a particular piece of DNA to prepare for detailed study and analysis. Cloning vectors, which can carry inserted restriction fragments, come in different types with different virtues and 
different problems. The simplest vectors are plasmids, small, circular pieces of DNA that are not part of a chromosome. The earliest ventures in cloning used bacterial plasmids to make copies of small pieces of foreign (non-bacterial DNA). Plasmids will accept inserts of about 5,000 to 12,000 bases in length $(5 \mathrm{~Kb}-12 \mathrm{~Kb}$; DNA lengths are standardly given in kilobases $(\mathrm{Kb})$ or megabases $(\mathrm{Mb})$ ). Bacteriophage, viruses that infect bacteria, can also be used as cloning vectors. One very important hybrid vector consists in modifying bacterial plasmids with special segments of bacteriophage DNA (so called cos sites) to produce cosmids. Cosmids propagate as viruses and will accept longer inserts than plasmids, without forfeiting the important virtue of stability (it is important that when the DNA is replicated pieces of the insert are not lost or rearranged); cosmids can be used to clone $30-50 \mathrm{~Kb}$ of foreign DNA. To clone even larger pieces of DNA, molecular biologists use a different vector, yeast artificial chromosomes -- or YACs. Essentially, YACs consist of a very long segment of foreign DNA, derived from some organism of interest, such as a human being, joined to crucial pieces of DNA from yeast which play major roles in chromosomal stability and segregation, so that the composite behaves like an entire yeast chromosome. When inserted into a yeast cell, a YAC is replicated along with the real (non-artificial) yeast chromosomes, and as the cell divides to form a colony, many copies of the long piece of foreign DNA can be obtained. YACs can be employed to clone segments up to a megabase in length (one million bases), although there are sometimes problems both in avoiding the insertion of noncontiguous pieces of foreign DNA and in guarding against modification of the DNA as the YAC replicates. Despite these difficulties, YACs are major tools in the investigation of the DNA of many organisms (including human beings).

Restriction enzymes can be used to digest an entire chromosome or even a whole genome, yielding a collection of fragments that can be inserted into cloning 
vectors. The result is a library -- either a chromosome library or a genome library - a set of clones (typically, cosmids or YACs), each of which contains a distinctive piece of the chromosome or genome. Scientists can keep track of the individual clones in the library to the extent that they can identify distinctive features. How is this accomplished? Bacteria or yeast can be grown to form colonies, and, if these colonies are treated with special solutions, their DNA will unwind. If a DNA (or RNA) segment whose sequence is complementary to a sequence in the clone of interest is added, then the new segment will bind to that clone, and if the segment (the probe) is appropriately tagged (for example with a radioactive atom), it is possible to identify the clone. Molecular biologists have an entire arsenal of similar tricks to find the clones they wish to investigate.

One important part of that arsenal consists in the ability to manufacture segments of DNA that are complementary to chosen pieces of RNA. In some organisms, retroviruses, the genetic material is RNA instead of DNA, and these organisms replicate by invading a host cell, forming a DNA complementary to their RNA which is then inserted into the host DNA, letting the host synthesize large amounts of viral RNA and proteins, packaging these into new retroviruses which then bud off from the host cell. The trick of turning RNA into DNA is performed by a special enzyme, reverse transcriptase. Now suppose that we take a cell from any organism, a muscle cell from a mouse, for example. Some of the mouse genes will be expressed in that muscle cell, and those genes will be represented in cellular mRNAs. We can use reverse transcriptase to produce complementary DNA ( $C D N A)$, and we can envisage doing this systematically to generate a CDNA library, for a particular type of cell in an organism, or even for the whole organism (although, as will become apparent later, there are serious worries about the completeness of any such library). The cDNA clones in the library (ideally) correspond to all the coding regions (either all the 
genes expressed in the cell or all those in the organism's genome), so that this appears initially to be a promising way to find all the important parts of a genome. Of course, in eukaryotes, where mRNAs are spliced prior to translation, the cDNA will not correspond to a continuous stretch of the genome; it will consist of all the exons stitched together, and the introns will be unrepresented (see Figure 2.7).

\section{$2.4 \quad$ Mapping}

The first major goal of the HGP is to map the human genome. This process will involve constructing maps of two quite different types. Genetic maps, are diagrammatic representations that show the relative locations of genes or markers along a chromosome - or for all the chromosomes of an entire genome. As already noted, genetic mapping in nonhuman organisms is almost a century old. The difficulty of dealing with many organisms, including our own species, results in considerable part from our inability to specify genes or other genomic markers (and, in our own case, there is the further complication that, even when we can identify genes, matings -"crosses" - cannot be arranged at will). One of the great breakthroughs in genetic mapping occurred when it was recognized that restriction enzymes can be used to produce genetic markers and that these can be employed to locate genes.

Suppose we have a DNA library for a human genome. If we digest a clone from the library with a new restriction enzyme (one not used in producing the original library), we will obtain a collection of fragments, each of which corresponds to one of the enzyme's preferred stretches of sequence within the DNA segment with which we began. Imagine now that we follow the same procedure on the corresponding piece of DNA from the genome of someone who has a mutation at one of these preferred stretches of sequence (recognition sites). This person's DNA will be chopped up differently: there will be one long fragment, instead of the two shorter ones found in 
the first place. (See Figure 2.8). These differences provide extreme examples of restriction fragment length polymorphisms (RFLPs). RFLPs can be treated like alleles that undergo the simplest type of genetic transmission. Most RFLPs involve more subtle changes in the length of restriction fragments, which come about because of insertions or deletions outside the recognition site -- in which case there will be the same number of fragments but they will have different lengths - but it is easiest to understand the general conception by focusing first on the most dramatic cases in which the recognition site itself is modified. Already, thousands of RFLPs have been found in human beings (as well as in other organisms, such as mice, fruitflies, and worms), and by studying the transmission of RFLPs through human genealogies (or by making standard genetic crosses in other organisms), it is possible to localize RFLPs to chromosomes and to discover their relative positions. Even more importantly, as we shall see, it is possible to situate genes with respect to RFLPs, which serve, in effect, as landmarks within the genome.

RFLPs are amenable to study because there are ways of identifying the relative sizes of DNA molecules. The fundamental technique, gel electrophoresis, consists in allowing DNA molecules to migrate through an agarose gel matrix under the influence of an electric field. (DNA molecules carry a negative charge, and move towards the positive pole). Smaller molecules move faster than larger ones. After allowing enough time for the molecules to separate, the current can be switched off, the gel treated to unwind the DNA strands, and a radioactive probe employed to pick out the DNA fragments of interest. So it is possible to see that one of the original sources of DNA, set into one of the lanes of the gel, has produced a single large fragment that has not travelled far, while the other has produced two shorter fragments that have gone further. (See Figure 2.9). 
The use of RFLPs (and other markers) has enabled molecular biologists to make rapid progress on a genetic map of the human genome. Yet, as noted above, genetic maps are only one kind of map. So-called physical maps are also important. A physical map of a chromosome or of an entire genome consists of an ordered cloned DNA library for the chromosome or for the genome, respectively. When we have constructed a library for a chromosome, we have shattered that chromosome into a large number of small pieces: the physical map puts them all back in the right order. More exactly, a physical map takes the libraries associated with several different methods of digesting the chromosome and situates them with respect to one another, so that the chromosome is covered in different ways with overlapping fragments (see Figure 2.10). This may be done at low resolution (using YACs), or in far more detail (with cosmids). A physical map with extraordinarily deep resolution might use a very large number of cosmids, each of which protrudes just a short distance (2-3Kb) beyond its neighbor.

\subsection{Sequencing}

The "ultimate physical map" is the sequence, the representation of the order of bases along a stretch of DNA, a gene, a chromosome, an entire genome. During the 1970s, two separate techniques for sequencing DNA were developed. Both employ gel electrophoresis to separate DNA fragments, differing in length by only a single base, and develop clever strategies for "reading" that additional base. In the "chain termination method", developed by Fred Sanger, a single strand of DNA serves as the template for building a complementary strand. The process occurs in a solution containing enzymes required for DNA synthesis and a supply of bases, some of which are normal, others of which ("dideoxy bases") can be incorporated into a growing DNA strand but will not allow the strand to grow beyond them: they terminate the chain. If this process is carried out four times, each time with a different dideoxy base 
(A,C, G or $T$ ) radioactively tagged, and if the results are run in four different lanes of a gel, the fragments will be spread out, and the radioactive tags will display the order in which bases have been added. (See Figure 2.11).

During the 1980s, a number of technical advances were made in sequencing. One important development simplified the process by tagging bases with different fluorescent dyes, so that the fragments can be separated in a single lane of a gel, revealing a distinctive array of colored bands that is readable by machines. A single lane of a gel can now be used to sequence a DNA segment of 500 bases). Where, in the early 1970s, Sanger, Walter Gilbert and Allan Maxam (the three original developers of DNA sequencing) labored to produce a hundred or so bases of reliable sequence over a period of months, small-scale sequencing is now a matter of routine, and many laboratories can produce several kilobases of reliable sequence in a day. However, it is extremely important to understand that scale matters. The human genome is estimated to contain 3 billion base pairs. Even with automated sequencing and the other refinements of contemporary technology, the task of producing the entire sequence is daunting. (The publication of the achievement of $2.2 \mathrm{Mb}$ of contiguous, finished sequence in the nematode worm, C. elegans (R. Wilson et.al. 1994) is a major accomplishment that has rightly inspired admiration among molecular geneticists.)

Another major advance of the 1980s was the invention of a method for producing large numbers of copies of DNA segments (amplifying those segments of DNA) without using cloning vectors. The polymerase chain reaction (PCR) takes advantage of the fact that formation of a complementary strand of DNA requires not only the appropriate helper enzymes, but also a small piece of the new strand (a primer). So, if the sequences adjacent to a piece of DNA one wants to copy are known, one can take a larger DNA fragment, unwind it to obtain single strands of DNA, add the primers so that they anneal to the sequences flanking the piece being 
copied on both sides, add the enzyme for initiating transcription, and produce new double-stranded DNA. Repeating this process through many cycles, it is possible to obtain a very large number of copies of the desired piece of DNA (see Figure 2.12).

\subsection{The State of the Art}

All the tools so far described are put to work in the HGP, and in related molecular research. At present, there are intensive efforts to produce physical maps of parts of the human genome, and to correlate these with genetic maps. Many of the smaller human chromosomes are well on the way to being covered with identified clones (for example, the study of chromosome 19 currently being carried out at Lawrence Livermore Laboratory by Anthony Carrano and his associates), and there is a rough map of the entire genome (Cohen et.al. 1993). Analogous efforts to map the genomes of the mouse, the fruit fly, the nematode, yeast, and other "model organisms" are even further advanced. Although there are difficulties, especially in finding landmarks in regions of genomes that have large stretches of repetitive sequence, molecular biologists have little doubt that it will be possible to complete genetic and physical maps to a high degree of resolution, within the period of the HGP.

The maps already developed are proving their worth in the identification of human genes, especially those genes that have pronounced effects on human health. Newspaper reports make clear the fact that the pace of gene identification has greatly accelerated during the past years. The reasons for this are not hard to understand. Dense maps facilitate a strategy, positional cloning, which biomedical researchers can deploy to identify and sequence genes that play a role in particular diseases. Starting with the recognition of a pattern of disease transmission within a family, scientists look for markers that are associated with the disease. Finding these markers - ideally markers that flank the disease gene closely -- they can use the correlated genetic and 
physical maps to pick out a small collection of YACs (or a larger collection of cosmids), on which the gene lies. Using a variety of tactics, they can then "explore" this DNA, looking for candidate genes. Candidates can be tested by trying to discover differences between the alleles present in healthy people and those who suffer from the disease, by tracking the effects of mutant alleles in model organisms, or by a combination of both methods. Once the gene has been found, it can be sequenced using the standard techniques.

Constructing the "ultimate physical map" by discovering the sequence of a human genome is more difficult. The naive thought that one should start at the tip of one chromosome, sequence 500 bases, move on to the next 500 , and keep going through one from each pair of autosomes (chromosomes that are not sex chromosomes), plus both the $\mathrm{X}$ and $\mathrm{Y}$ chromosomes (the sex chromosomes), presupposes that we have a map of the entire genome that resolves it into segments of no more than $\mathbf{5 0 0}$ bases. Obtaining a map at such an extraordinary degree of resolution would be highly problematic. Current strategies for generating large stretches of sequence proceed differently. They start by chopping up a large piece of DNA into smaller fragments, and do this in many different ways. The bits and pieces are then sequenced, and the total sequence is reconstructed by finding overlaps, effectively solving the problem of the entire sequence as if one were doing a jigsaw puzzle. So-called "shotgun sequencing" avoids the labor of beginning with very detailed mapping, but it does involve considerable redundancy. (In most current sequencing efforts, the same segment of DNA is sequenced between seven and ten times.) Producing reliable, finished, sequence is by no means a mechanical process, and it is especially complex in those regions of the genome in which there are long repetitive stretches (where, for obvious reasons, it is difficult to work out exactly how the small pieces of sequence fit together). One important, intermediate, goal of the 
HGP is to devise more refined approaches to sequencing that will enable sequencing to go forward at faster rates (and more cheaply). The hope is that, by the end of the project, sequencing will be as routine as the manufacture of equipment has become (Gilbert 1992).

For lucid presentations of the material of this section, together with vastly more detail, the reader should consult either of two excellent texts: (Berg and Singer 1992) or (Watson et.al. 1992); the latter is more advanced and comprehensive. 


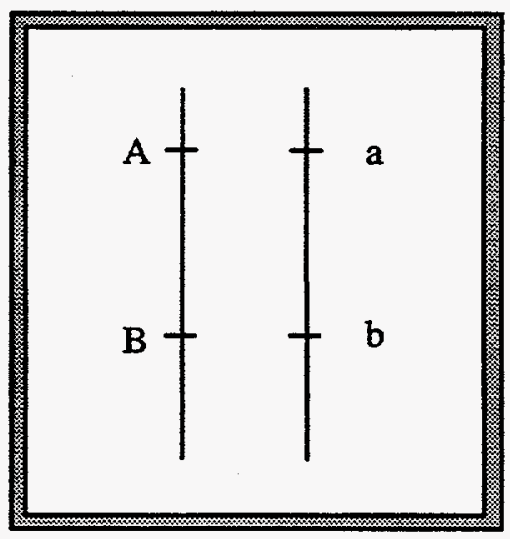

Fig. 2.1 


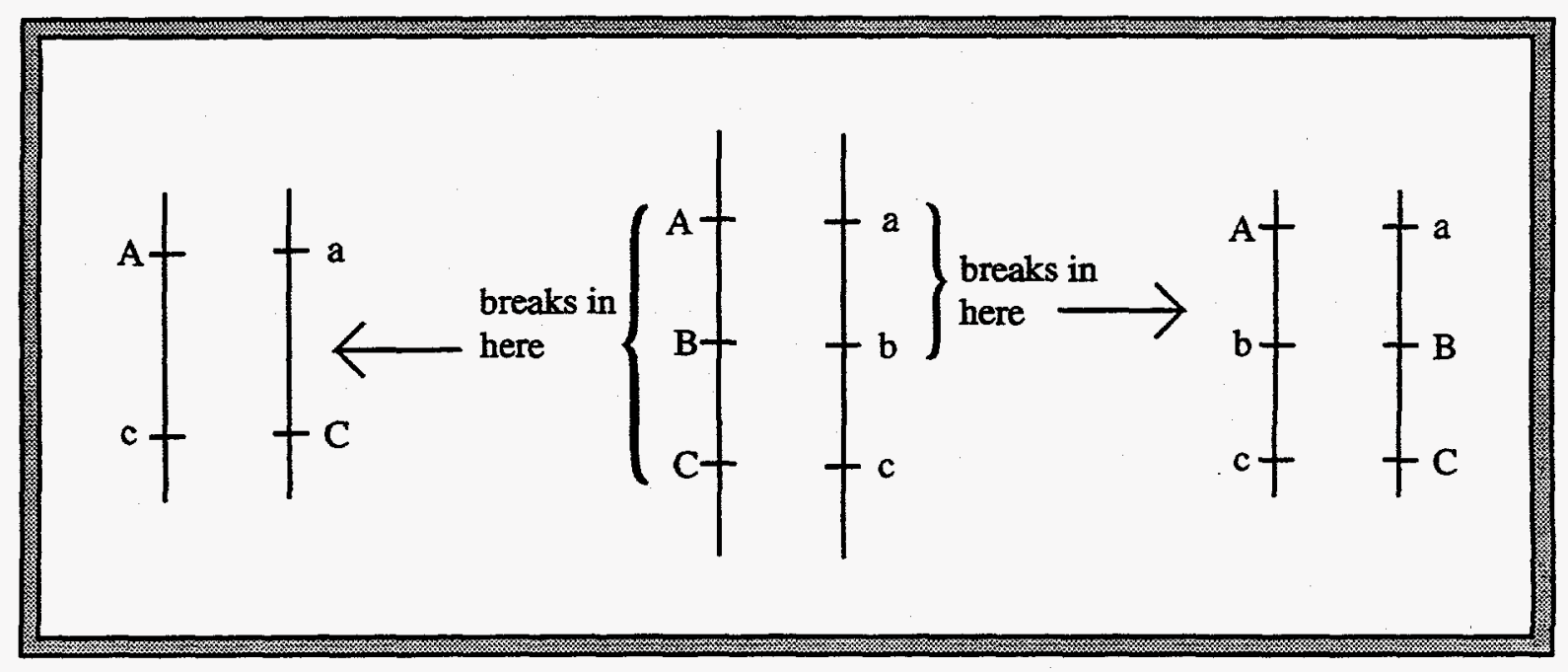

Fig. 2.2 


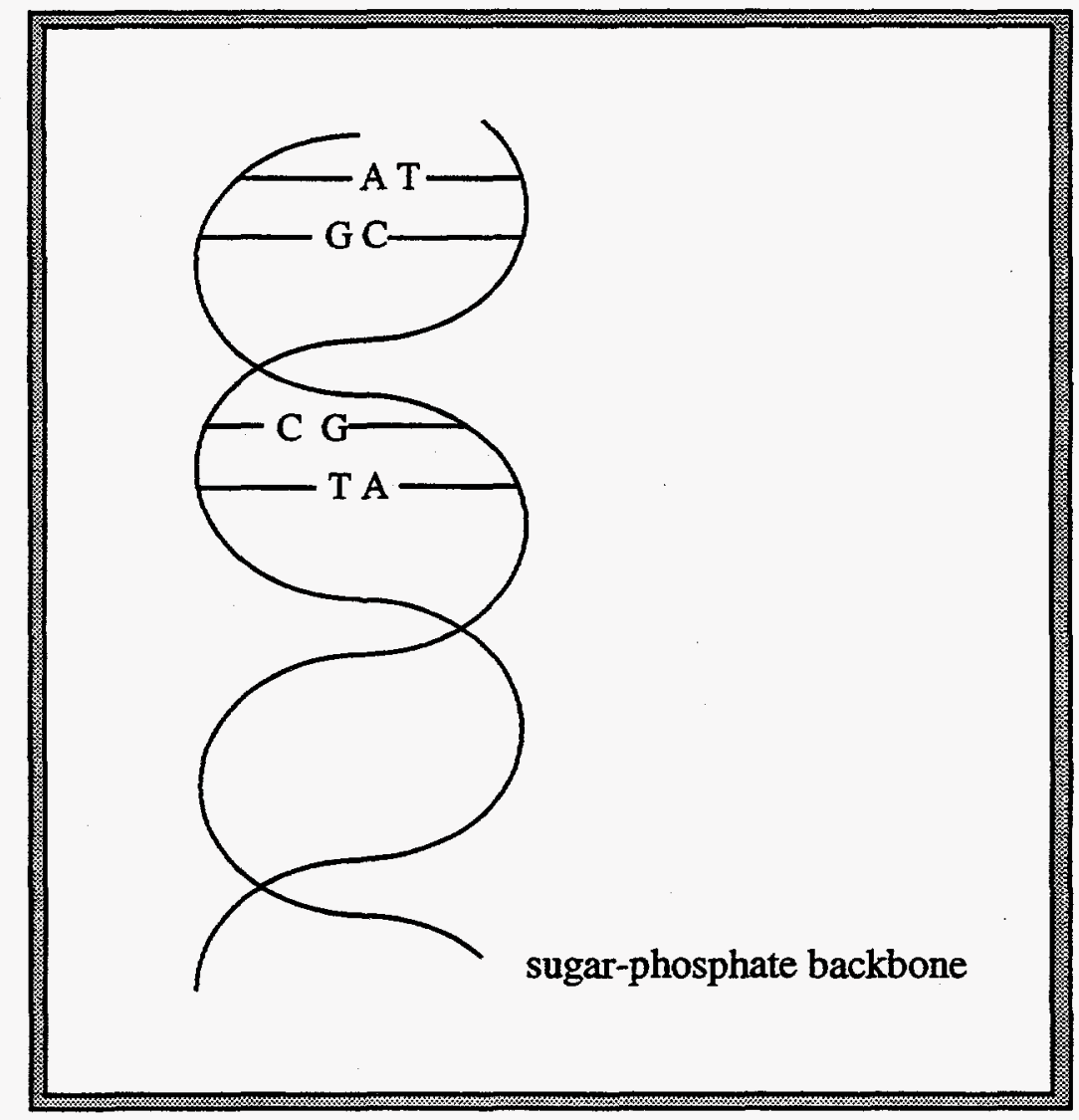

Fig. 2.3 


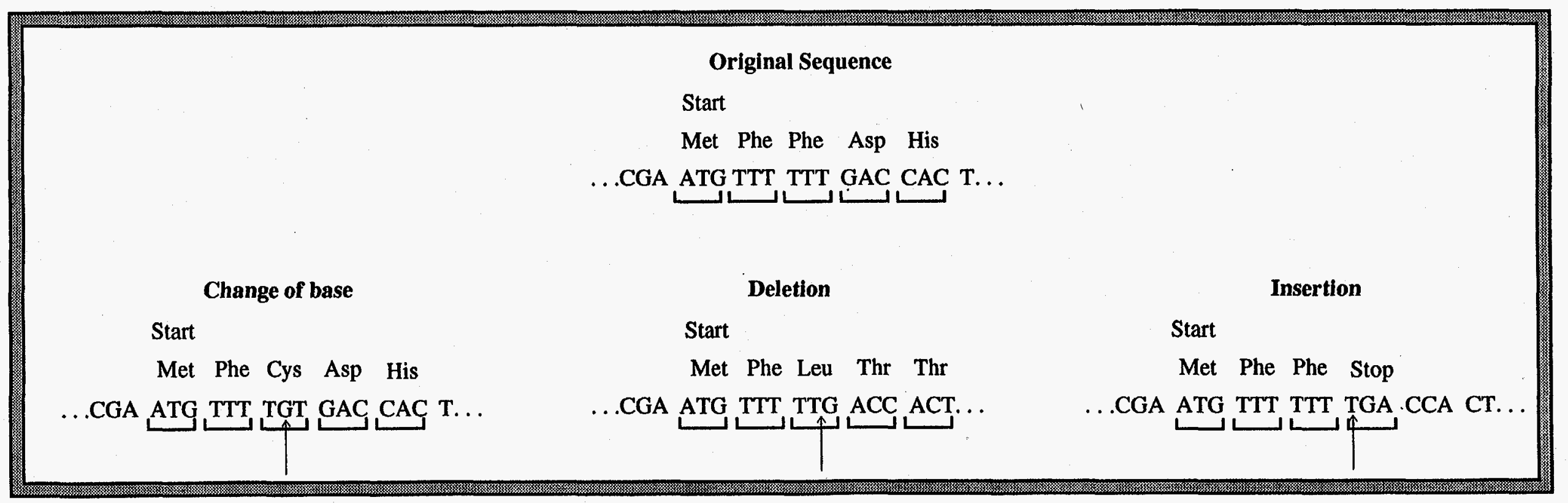

Fig. 2.4 


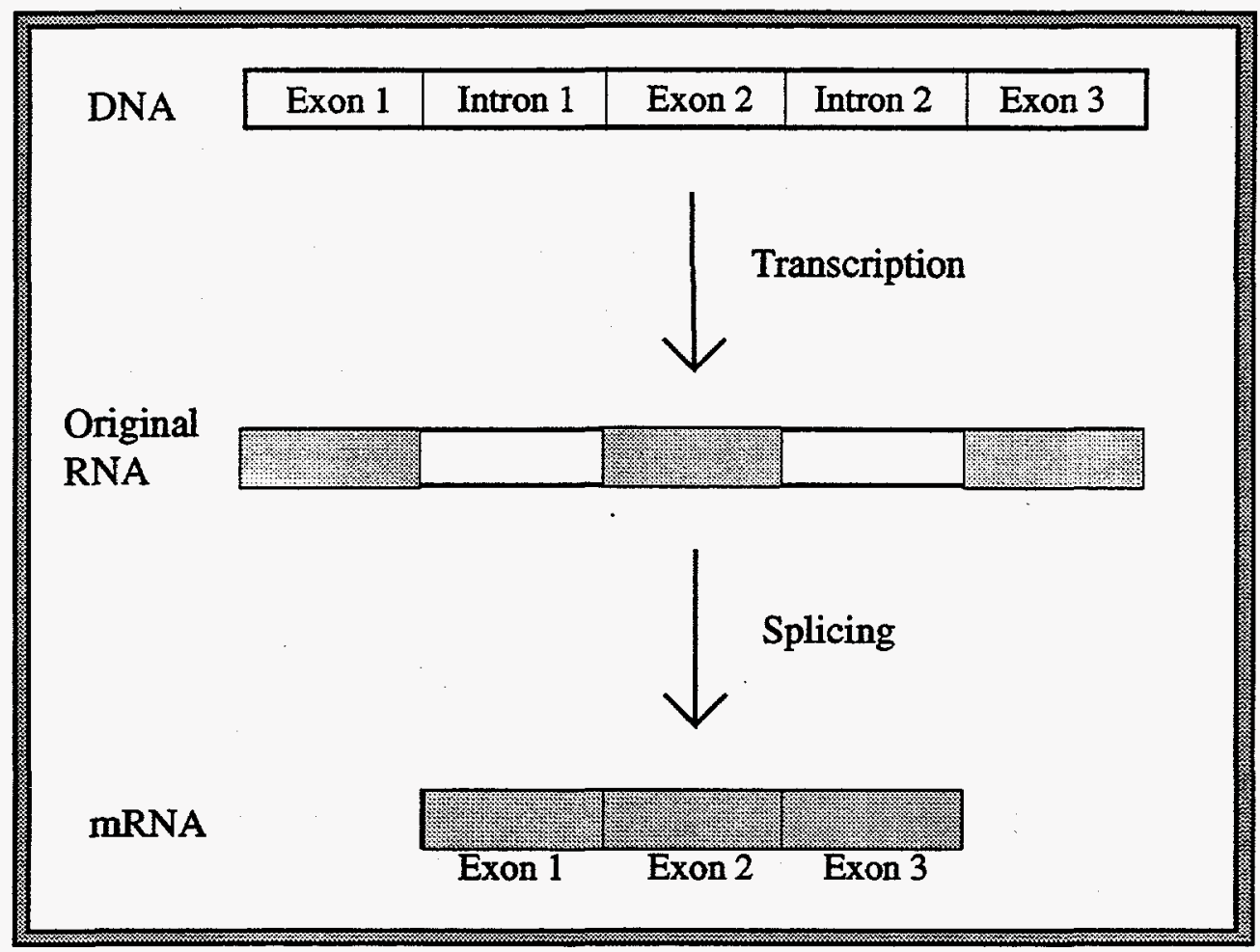

Fig. 2.5 


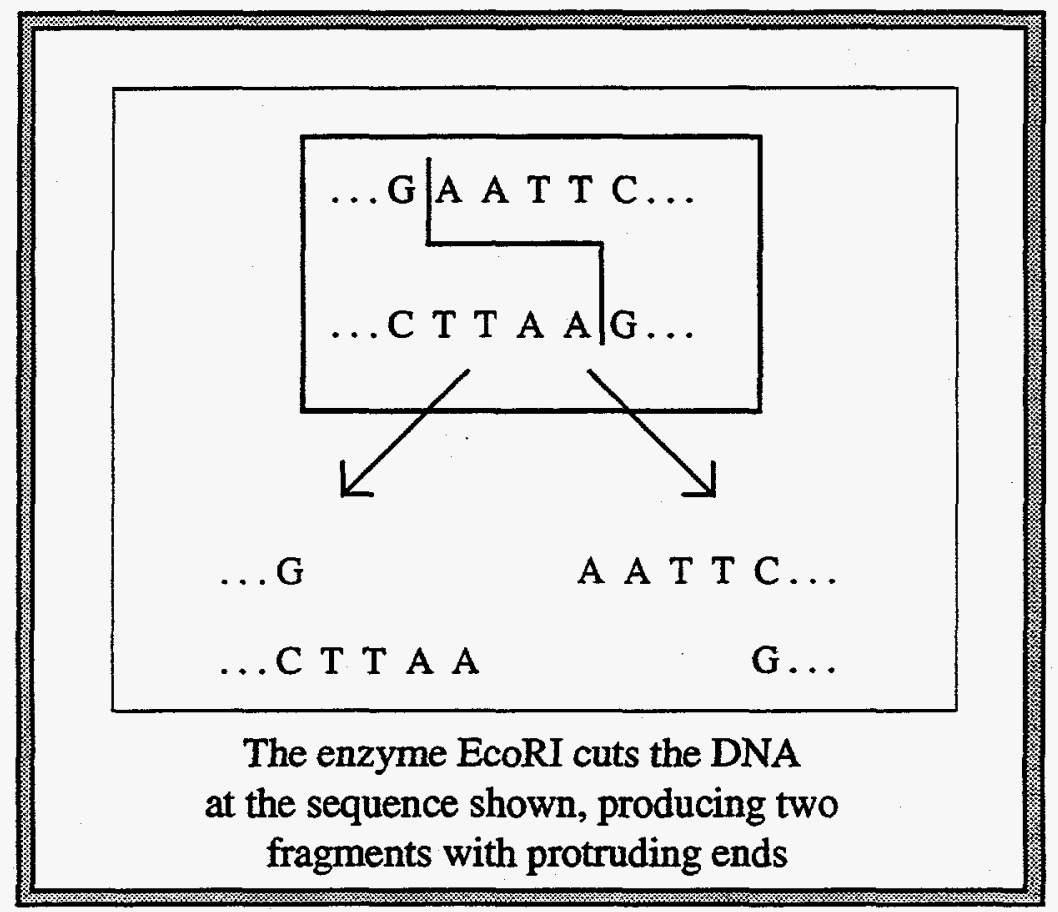

Fig. 2.6 


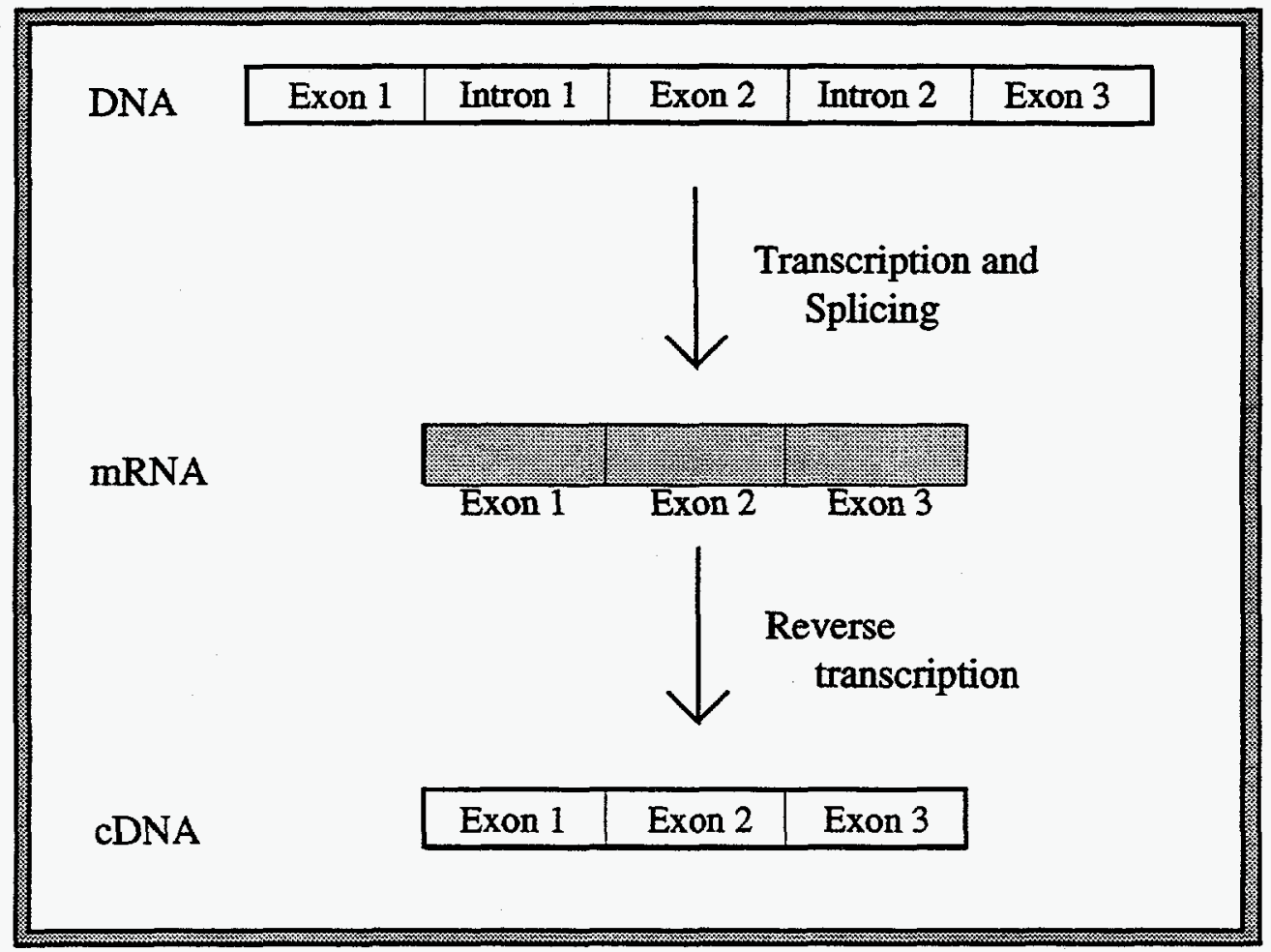

Fig. 2.7 


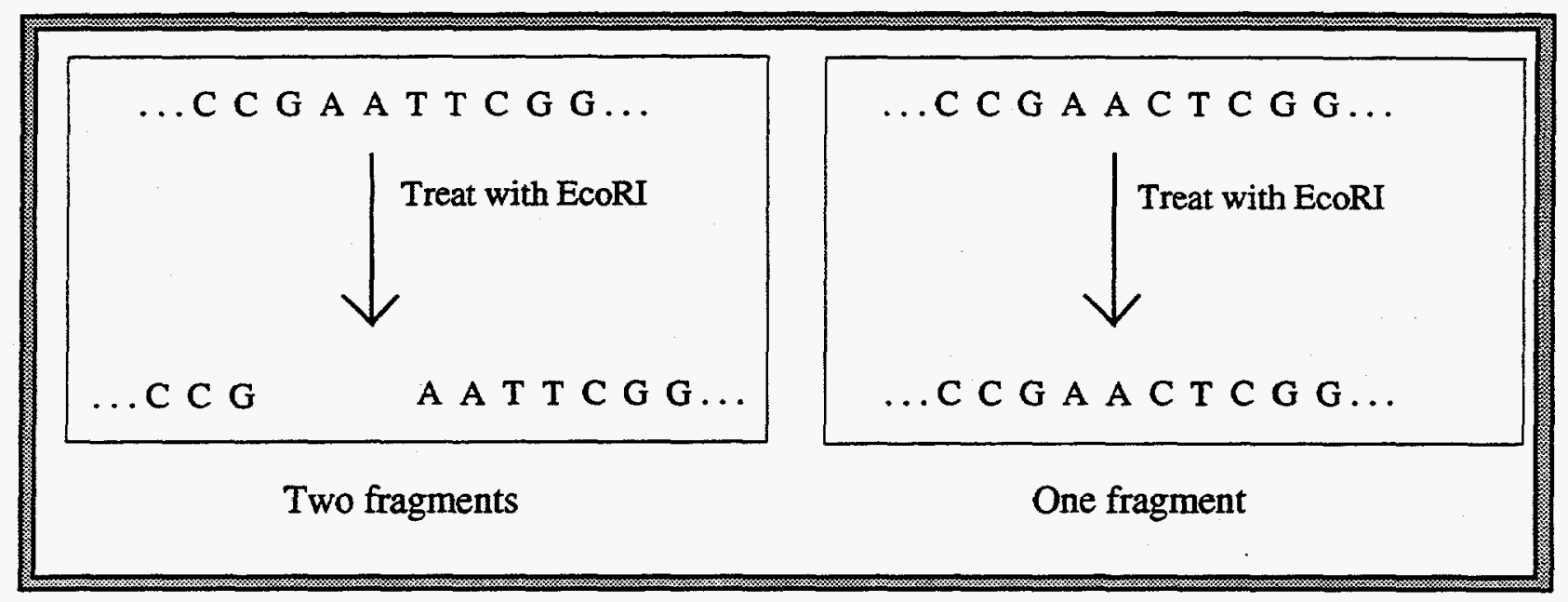

Fig. 2.8 


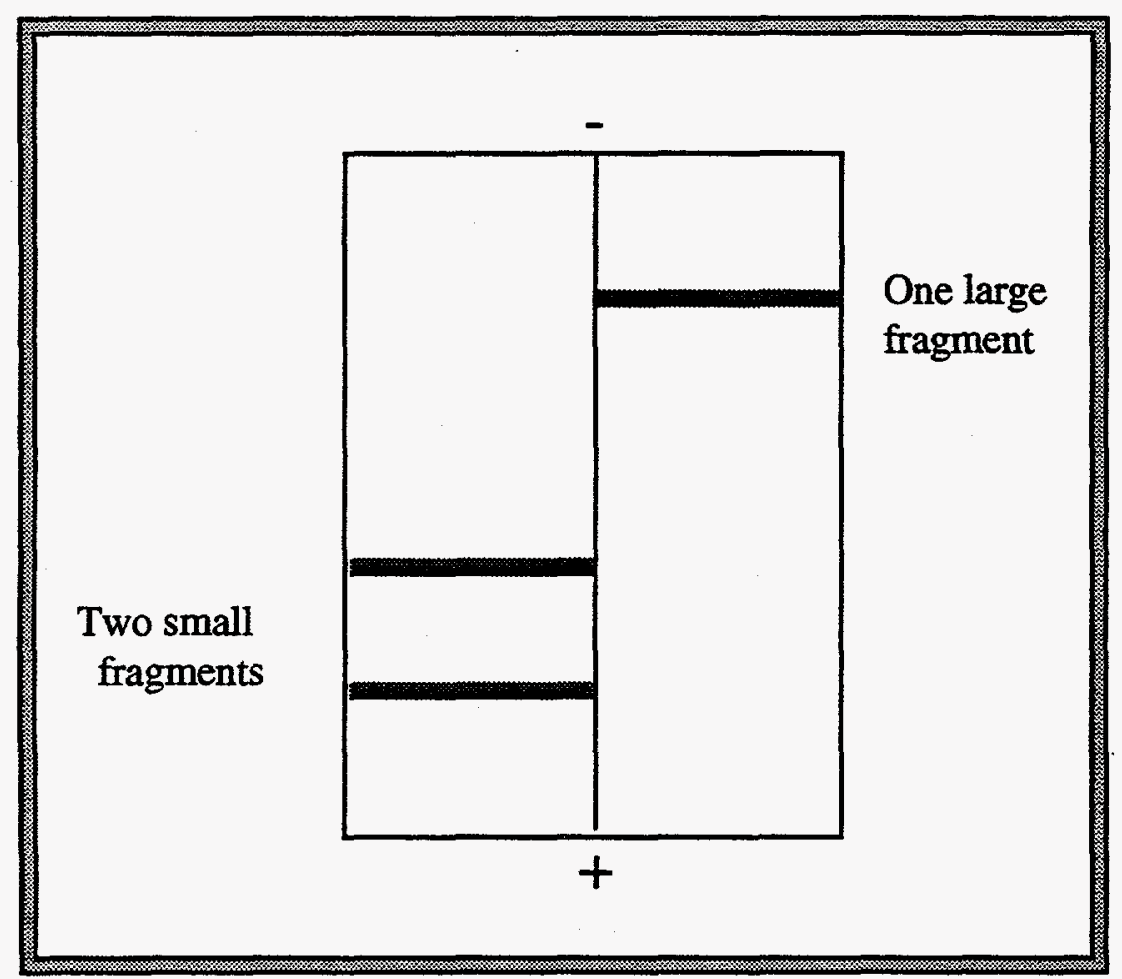

Fig. 2.9 


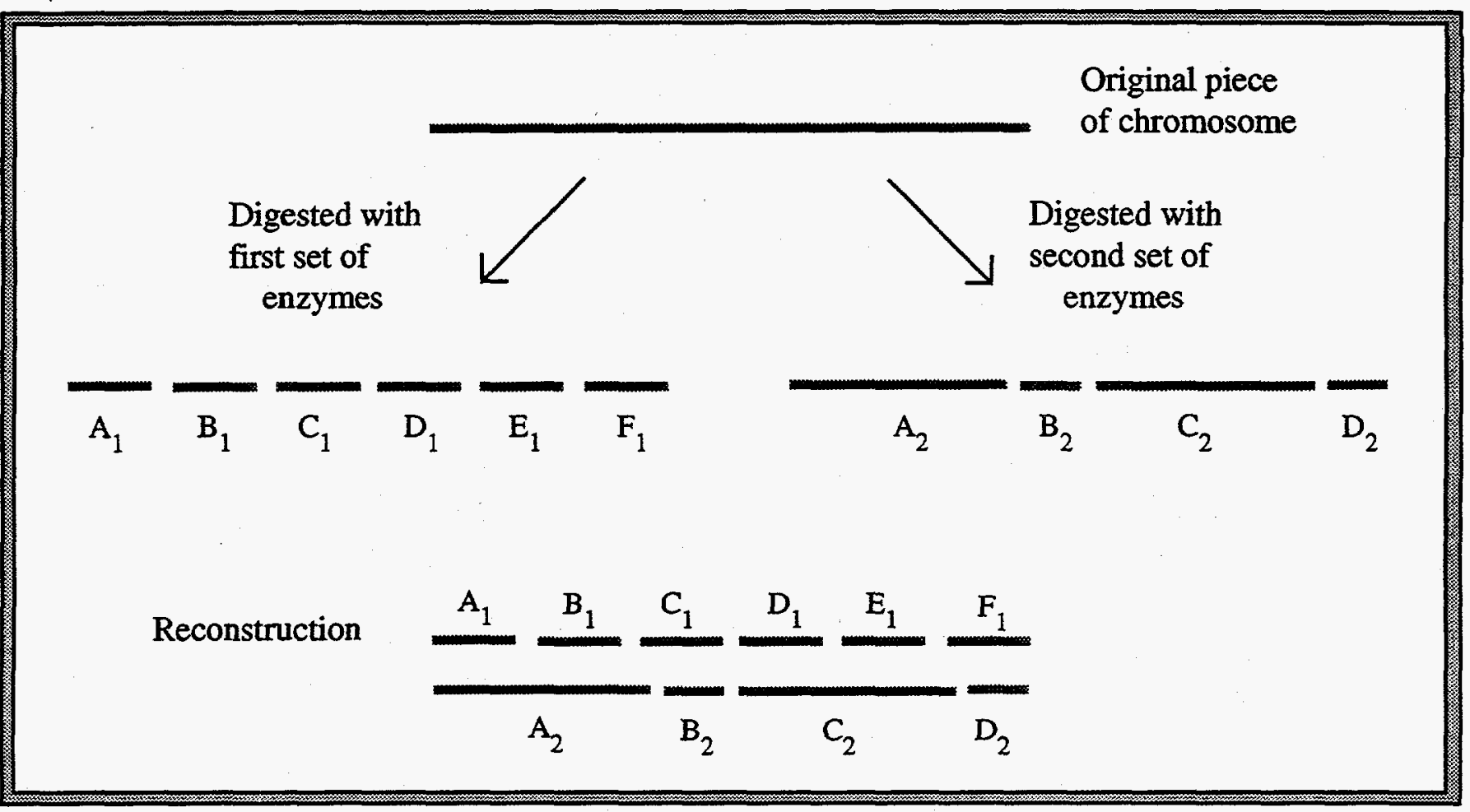

Fig. 2.10 

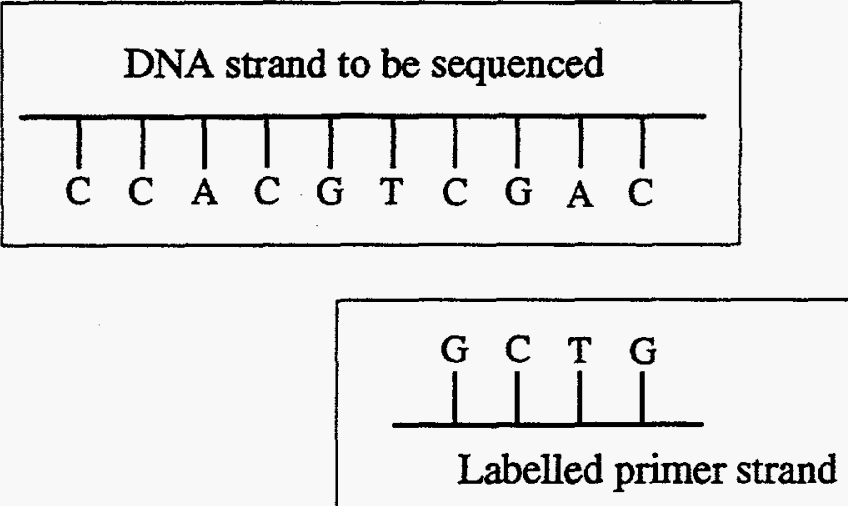

Helper enzyme +4 normal bases + labelled dideoxy thymine

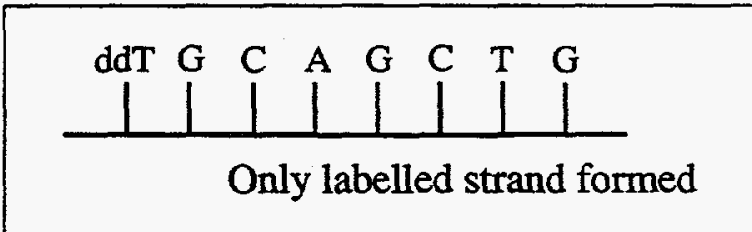

Repeading this using labelled dideoxy versions of the other bases, the strands are separated in different gel lanes:

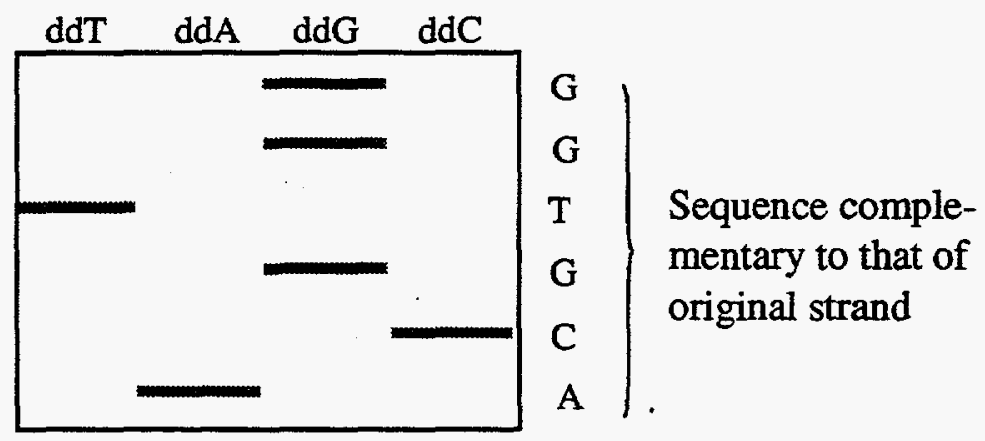

Fig. 2.11 


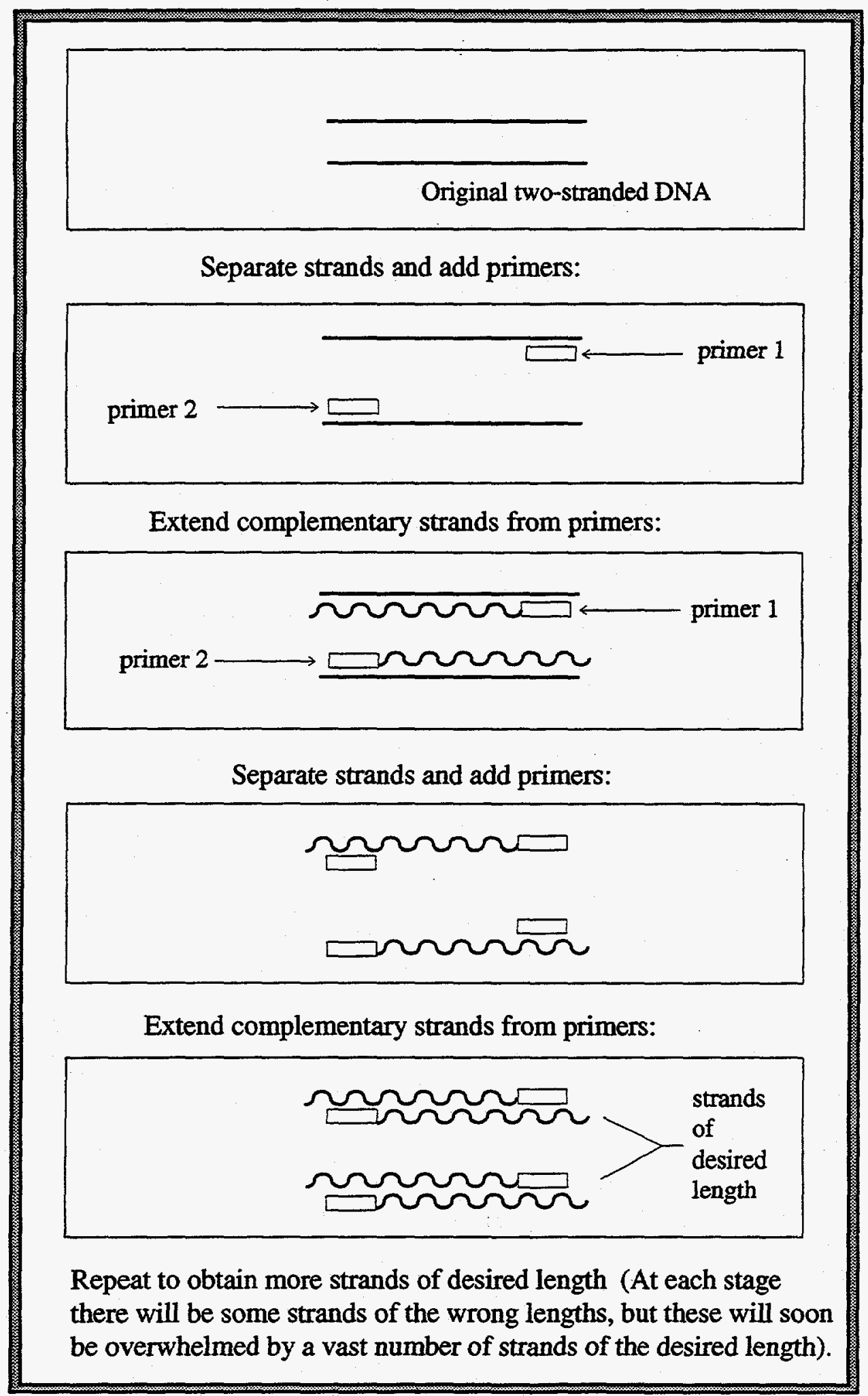

Fig. 2.12 


\section{The HGP and Beyond}

\subsection{Conflicting Claims about the HGP}

Walter Gilbert has described the HGP as the quest for "the grail" of biology, and other champions of the project have waxed equally enthusiastic, claiming that mapping and sequencing the human genome will be a major step forward in biology as well as providing important medical and biotechnological applications (Gilbert 1992). Critics of the project counter by denying that the HGP has the scientific significance attributed to it, by suggesting that the applications are either unattainable or else do not bring the benefits with which they are credited (Lewontin 1992, Hubbard and Wald 1993). The task of this section is to review and evaluate claims and counterclaims about the scientific significance of the project.

Relatively little controversy surrounds the biological point of constructing more detailed genetic and physical maps of the genomes of human beings and other organisms. A host of successful recent ventures in gene-hunting testifies to the increased power that the new maps have brought (for example, the discoveries of genes implicated in cystic fibrosis, Huntington's disease, and colon cancer). Although the path from a segment of DNA containing a number of candidate genes to identification of the gene sought can be tortuous, and although there are subtle difficulties in cases in which the phenotype results from many genes acting in concert (so-called polygenic traits), it would be hard to gainsay the value of maps in identifying genes. The serious worry concerns the value of applying the knowledge of human gene sequences in medical contexts: as we shall see in section 4 , there are important questions about the value of genetic tests.

Genomic sequencing is quite another matter. The last section noted that the size of the human genome ( 3 billion base pairs) makes the task of constructing the entire sequence extremely difficult. Highly talented, imaginative scientists are needed to direct the coordinated parts of a massive sequencing effort. What exactly will be 
gained from this deployment of great intellectual resources? Even with the investment, will the sequencing be feasible? Although it should be obvious that the last question is an important one, discussion of it will be deferred until we have a clearer view of the benefits that a complete sequence for the human genome might bring.

Assessing the worth of the envisaged complete sequence is impossible unless we look beyond the project to the biology that it will make possible. Gilbert has defended the HGP by depicting a royal road to biological understanding. Given the sequence, our first task is to catalog all the genes; armed with a list of genes, we use the genetic code to find the proteins for which they code; this yields a representation of proteins as sequences of amino acids, and the next task is to understand the three-dimensional structure and function of those proteins; finally, we shall try to work out the interactions among proteins and nucleic acids in cells, to uncover the fine details of physiological processes, and eventually to analyze the causal pathways that underlie development and metabolism.

Other defenders of the HGP are far more pragmatic. David Botstein has compared the enterprise of sequencing the human genome to that of building a superhighway system, along which twenty-first century researchers will travel. But it is important to understand why anyone will want to use these highways. Gene sequences are apparently valuable to all kinds of biological investigations, but the genes are sparsely distributed in the human genome (and in mammalian genomes generally). It is estimated that we have between 30,000 and 100,000 genes; if these genes, plus their flanking regulatory regions average about $3 \mathrm{~Kb}$, then they amount to at most $300 \mathrm{Mb}$ in a genome of $3,000 \mathrm{Mb}$, or about $10 \%$. Why is it important to sequence the remaining $90 \%$ of the genome?

The most obvious reason for sequencing the entire genome is that it will enable us to identify all the genes, so that we can start a systematic investigation of the 
interactions among gene products. However, there appears to be a simpler strategy. As explained in section 2, we could isolate the mRNAs that are found in the cells of an organism, make corresponding cDNAs, use these CDNAs to pick out the chromosomal DNA (a process that is not completely straightforward, but which is simpler than mass sequencing), and then sequence the chromosomal DNA (and some stretches of flanking DNA) in conventional fashion. This strategy might lead us to overlook important features of genome organization or noncoding regions that are involved in the regulation of genes, but the most significant criticism of it centers on worries that it will not yield a complete catalog of genes (see, for example, Olson 1992).

There is no doubt that searching for mRNAs will direct us to the most popular genes, those that are switched on for significant periods in readily accessible cells. However, there are bound to be some genes that are only expressed for brief periods in a handful of cells. It might be supposed that these are not very important, but this would be mistaken: some of these genes direct the formation of proteins that help to regulate the activation of other, more prominent, genes. Understanding normal and abnormal development will require us to identify their fleeting -- but crucial -contributions. If we are not to omit them from the catalog, then it will be necessary not only to sample widely among the cells of adult organisms, but to be equally thorough at all stages of development from zygote on. Assuming that fetal tissues are available for research, biologists are not completely blocked from finding the mRNAs that appear briefly in development, but, without breaches of common ethical standards (for example, by letting artificially fertilized eggs mature to various stages in a massive and systematic program), it will be impossible to have any confidence that all the human genes have been identified. Ironically, although the HGP has been charged with "blind reductionism" (Lewontin 1992, Tauber and Sarkar 1992), it is the antireductionist insistence of the project that we prepare for studying interactions by picking out all the molecules involved that underlies the search for all the genes, and, 
consequently, the preference for full sequencing rather than a less systematic approach via mRNAs and cDNAs.

Finally, there is a pragmatic point. The challenge of mass sequencing can be expected to act as a spur to further improvements in sequencing technology, making large-scale sequencing trivial, and this would prove of immense value to the biology of the twenty-first century. Even if that is over-optimistic, less dramatic advances -attaining rates of sequencing that made the task of generating a full human sequence manageable but not trivial - might still support the claim that complete sequencing is the most efficient way of finding all the genes.

This presupposes that complete sequencing will provide $a$ way to discover all the genes, and that the discovery can be connected with significant projects for the biology of the next century. Both claims have been disputed, and understanding the issues involved is important for assessing the scientific worth of the HGP.

\subsection{Sequence and Beyond: The Role of Model Organisms}

There are two main techniques available for moving from sequences to genes. One is to search a region of sequence for open reading frames, strings of bases that continue a long way without a stop codon. In each chunk of DNA, six reading frames are available: there are, of course, two strands, which would, potentially be transcribed and translated in opposite directions; on each strand, we can envisage starting at the first base, the second base, or at the third base; because triplets of nucleotides code for

amino acids, starting at the fourth base is equivalent to beginning with the first, starting at the fifth tantamount to beginning at the second, and so on. Computer analysis of regions of the genome can direct our attention to areas in which there are long open reading frames.

Another method, currently more popular, is to build on the knowledge we already have. Sequences of thousands of genes in $E$. coli, in yeast, in nematodes, in 
fruitflies, in mice, and in our own species, are available in data banks. We can obtain many more sequences by using cDNA analysis, especially on organisms that we can manipulate (thus overcoming the difficulties noted above). Using algorithms that seek similarities in sequence pattern (and thus indicate homologies, sharing of sequence through common evolutionary history), regions of DNA can be explored, and other likely genes identified. As more genes are discovered in this way, the data bank itself becomes larger and the process can be iterated. The power of existing algorithms is subject to check. By taking a well-understood organism, where we have confidence that we have been able to identify all the genes in a particular region of the genome (perhaps by finding the mRNAs), we can perform a computer experiment. Starting with the sequence data for the region and a modest data base of gene sequences, we can run the computer algorithm, iterate the procedure until nothing new is added, and then examine the extent to which all the genes have been identified. This procedure will both enable us to check algorithms, and to refine them.

Gene hunting by way of homologies takes advantage of one of the fundamental characteristics of the evolutionary process. The history of life plays all kinds of variations on a relatively small number of themes. New genes do not appear by some magical concatenation of bases in the genome, but are typically built from copies of old genes, modified, expanded, truncated or recombined. The bootstrapping procedure is biologically faithful, and we can hope to fine-tune our search procedures through detailed investigations of the organisms whose genetics we can explore most thoroughly (E.coli, yeast, nematodes, fruitflies, mice).

At present, it appears likely that we may overlook a small percentage of the human genes. None of the existing programs is well-designed to find genes with large introns, to uncover embedded genes, or to deal with a number of other known complexities of the genome. The best way of overcoming these difficulties is to concentrate on the nonhuman "model" organisms, and, if it is possible to refine our 
algorithms on their genomes, that knowledge will be applicable to obtaining an almost complete catalog of the human genes from the human sequence data.

From the structure of a gene (provided that we know how it is built up out of exons and introns), the genetic code gives a representation of the one-dimensional structure of the corresponding protein - the ordered sequence of amino acids out of which it is composed. To go further to the three-dimensional structure is notoriously difficult: the general problem of predicting how proteins fold remains unsolved, although crystallographic investigations enable the structures of some proteins to be identified. We can use this knowledge as a basis for further investigations, adopting a parallel strategy to that envisaged in the hunt for genes. Just as there are programs that seek sequence homologies, so too there are algorithms for identifying significant sharing of amino acid sequences. Many "new" proteins can be assimilated to proteins whose structures have already been identified by crystallographic methods, and, frequently, whose functions are known. Already it is sometimes possible to recognize a particular sequence as containing a coding region, and, virtually simultaneously, to specify the amino acid sequence, the three-dimensional structure, and the function of the protein. There are almost certainly limits to the success of this bootstrapping strategy, but, at present, there is reason to think that these limits are relatively broad, that careful investigation of the genomes of model organisms will enable us to specify the structures and functions of a large number of proteins, and that we shall be able to use this knowledge to understand a large number of human proteins.

In a narrow range of cases, a gene's causal contribution to the cell, a tissue, an organ, even the phenotype of the whole organism, can be inferred from knowledge of the structure and immediate function of the corresponding protein. Discovering that a gene codes for a DNA repair enzyme enable us to make educated guesses about the differences that that gene will make to the phenotype at various levels: this point has been dramatically illustrated in the identification of genes implicated in colon cancer 
through the recognition of similarities between a piece of human sequence and DNA repair genes in yeast. To track down the effects that differences in alleles at a locus produce in the phenotype -- and thus to understand the ways in which that locus affects the phenotype -- the most direct method is to make directed changes in the DNA in zygotes (or in selected tissues of an embryo), let development proceed in carefully controlled environments, and search for phenotypic differences. So-called "knockout" experiments would be grossly immoral on members of our own species (not to mention the difficulties posed by the confounding effects of many unknown environmental variables). To explore the relationships between genotype and phenotype, it will be necessary to study an animal that is close enough to ourselves to give us a significant chance of identifying the phenotypic effects of a significant number of our loci, an animal which breeds rapidly, whose genetics is relatively well understood and which can be reared in a variety of simple environments. We need the mouse.

Much significant information about genotype-phenotype relations can be obtained in this way, but it will be hard to deploy knockout experiments to probe the more subtle ways in which genes affect phenotypes. To supplement the strategy just sketched it will be important to analyze the causation of phenotypic traits in organisms whose development is easier to analyze than is that of the mouse. Biologists will have to turn to other organisms to obtain the developmental understanding that will guide their investigations into gene functioning in mice.

Three organisms are likely to prove especially useful. Studies of yeast have already revealed much about the basic processes that occur in all eukaryotic cells. Work on the nematode worm, Caenorhabditis elegans, will elucidate the molecular pathways that underlie the development of multicellular organisms, including the development of a rudimentary nervous system. Thanks to years of painstaking research by scientists at Cambridge University, there is a detailed "fate map" of $C$. 
elegans, a chart showing the "cell family history" of each cell in the adult worm; there is also a complete diagram of the neural organization of the nematode. Using these magnificent tools, it will be possible to proceed from knowledge of genes, proteins and their functions, to an understanding of the patterns of gene expression at various developmental stages and the molecular genetics of elementary neural functioning. In particular, researchers will attempt to pin down the exact points in development at which genes are expressed, using this as the basis both for recognizing the effects that mutations have on the adult phenotype and the main features of developmental pathways. Finally, during the past two decades, developmental studies of the fruitfly Drosophila melanogaster have shown how molecular genetics can be applied to show how the body plan is laid down in early larval development. Roughly one third of open reading frames in that part of the Drosophila genome that has so far been sequenced mutate to yield phenotypes that can easily be detected by the experienced fly biologist (Gerald Rubin, personal communication). Building on knowledge of protein structure and function, on information about the expression of genes in tissues, and on the growing understanding of developmental pathways, it will be possible to look for insights into where to look in the "undetectable" cases. Although the problems of analyzing development and metabolism are not trivial in either fruitflies or nematodes, both these organisms offer tools that are lacking in either mice or men. (For extensive further discussion, see Science 1988).

Other nonhuman organisms, too, may play an important role. It appears that coding regions in the soil ameba, Dictyostelium discoideum, are relatively easy to identify, so that sequencing the genome of this organism may be useful for refining our programs for searching out genes (William Loomis, personal communication). Similarly, in bridging the gap between flies and mice, it may be valuable to investigate the genome of a vertebrate: the puffer fish has been suggested as an appropriate candidate. But the moral of this brief account of the ways in which sequence data 
about human beings can be translated into deep biological understanding should be abundantly clear: the HGP needs the genome sequences of a number of well-chosen model organisms (5-10, including at least $E$. coli, yeast, $C$. elegans, fruitflies, and mice).

The importance of sequencing nonhuman genomes cannot be overestimated. Without the model organisms, the human sequence data would be scientifically barren, and the practical significance of those data greatly diminished. Diagnoses of mutant alleles would remain possible (in cases where we could identify genes using classical methods), but the aspirations to move beyond diagnosis to the rational design of therapies based on molecular understanding depend on assignments of gene function. Model organisms are not an afterthought to the HGP. They are at its center. (Not that the use of model organisms guarantees that we shall be able to interpret the human sequence data - for there may be significant disanalogies - but without those organisms there is virtually no chance of interpretation.)

Genomic sequencing seemed the best route to finding all the human genes -- and so it may prove. However, if we have a rich understanding of the smaller genomes of the model organisms, then the information they provide, coupled with that we can derive from human cDNAs, can be used to direct our investigations of the 3 billion bases of the human sequence. We may gain enough knowledge to probe the coding regions of the human genome, and round up all the human genes; more exactly, even without complete sequencing, we may be able to identify all those human genes whose functions we would be able to identify. Since, as we shall see below, there are serious concerns about the possibility of sequencing anything so massive as the human genome, it is encouraging to recognize that the HGP can accomplish an enormous amount in indirect, pragmatic, ways.

Approaching our own genome through those of worms, flies, and mice may seem unsatisfactory to those who hope that the HGP will reveal "what is distinctive 
about humanity" (Gilbert 1992). But the idea that genomic investigations would show us what makes us different from other species should always have seemed problematic. A point-by-point comparison between the human genome sequence and the sequence of chimpanzees (our closest evolutionary relatives) would be interesting, showing us the (probably small number of places) at which new alleles are present and where genes have been rearranged, possibly modifying the control of gene expression. That comparison would resolve some evolutionary questions about the birth of our own species. But most of those who pose the question "What is so distinctive about being human?" do not have recondite evolutionary questions in mind. They want to know what has made it possible for us to communicate with one another, to compose string quartets, write poems, paint frescoes, control sub-atomic sources of energy, and probe the genomes of living things.

Nothing in our sequence of As, Cs, Gs, and Ts is remotely likely to provide an answer to these kinds of questions. We probably share that sequence, to all intents and purposes, with many other beings who lived on our planet during the last hundred thousand years. Our human ancestors shared with us the capacity for culture, and it is possible that, within the next century, molecular biology, developmental biology, and neuroscience, will join to enable us to understand how the capacity for communication, attention, and sophisticated planning relates to features of our brains, how those brains develop, and how the (probably small) genetic differences between human beings and chimpanzees bear on the typical neurological development of the two species. Even if molecular biology, developmental biology, and neuroscience fulfil all their promise, help from still other fields will be needed if we are to understand how the capacity for culture has been expressed in complex and ramified ways -- if, that is, we want to know how (probably small) initial differences have been magnified during the past few million years. To obtain that knowledge will demand the resources of a mass of natural and social sciences, many as yet in their infancy. If the question of the 
distinctiveness of humanity is posed in the natural way -- the ambitious way that transcends the interesting, but limited, evolutionary question of the origin of our species -- it is not a question for biology alone.

The conclusion to be drawn is that the HGP has the potential to deliver an enormous amount of important biological information about ourselves. That information will be obtained through detailed comparisons of our genes with those of other organisms and through investigations of the functioning of genes in nonhumans. It is already clear that we share an enormous number of our traits with other organisms, and that studying those organisms can yield important insights about human physiology and human disease. Precisely the most difficult characteristics of ourselves to fathom will be those that are not identifiable (and manipulable) in model organisms.

\subsection{Can it be done?}

In section 2, and also earlier in the present section, the difficulties of mass sequencing were emphasized, and questions of the feasibility of the project were postponed. At present rates of sequencing, generating the full 3 billion base pairs of the human sequence is out of the question. Genome centers presently project to produce about a megabase of finished sequence per year (these estimates are for 1994; they are made by center directors; the scientists and technicians who do much of the daily work are usually more cautious, suggesting that the rate will be around $700 \mathrm{~Kb}$ ). At the rate of a megabase per year, 3,000 center years would be required to sequence the human genome alone. Assuming 30 centers, worldwide, with the necessary equipment and expertise, are assigned to human sequence, it would take a century to finish the job.

Genome scientists are confident that sequencing rates will improve, and they agree that they must improve at least tenfold, and ideally one hundredfold, if the project is to become manageable. Given the discussion in this section, it is evident 
that the ideal would be to sequence the human genome, the mouse genome, plus the genomes of several model organisms $(E$. coli is about $4 \mathrm{Mb}$, yeast about $15 \mathrm{Mb}$, the nematode around $100 \mathrm{Mb}$, Drosophila about $150 \mathrm{Mb}$, the mouse, like other mammals, about 3 billion bases). Plainly it would be worth sequencing the mouse genome if we can, and the mouse genome will be feasible if the human genome is. Sequencing the smaller genomes is less problematic: with envisageable improvements in sequencing technology, yeast and C. elegans should be finished by the end of the century, and Drosophila should be done by the time the HGP reaches its end in 2006. But unless sequencing technology undergoes dramatic improvements, the full human sequence may well remain beyond our reach. The initial argument for obtaining the whole sequence stressed that this would be an efficient way of identifying all the genes. That will only be true if there are very large advances in sequencing rates. (Considerations of rate are usually tied to considerations of cost; the costs of sequencing will need to be reduced if the full sequence is to be obtained within the project's budget; this will occur as a consequence of increasing sequencing rates.)

Where might these advances come from? Approaches to sequencing divide into two types, those that try to refine further the gel-based methods pioneered by Maxam and Gilbert and by Sanger, and those that attempt to read the sequence in a different way. Some ambitious proposals of the second type have been explored. One, based on the idea of letting a microscopic needle "feel" the tiny differences in forces associated with different bases (scanning tunnel electron microscopy) seems to have foundered on the difficulties of keeping DNA molecules sufficiently still. Another method that aims to snip individual bases from a DNA molecule and have them fall through a laser, designed to detect differences in fluorescence, has not so far fulfilled its early promise. Most (though not all) of those currently working in the genome project believe that the best hope for increasing rates of sequencing lies in improving the speed of gel-based methods. 
There are several suggestions for improvement. One is to abandon the "shotgun" approach in favor of a highly directed sequencing strategy based on a very detailed physical map. As remarked in section 2, if we had an ordered sequence of DNA segments, each protruding from its "downstream" neighbor by only $2-3 \mathrm{~Kb}$, it would be a relatively easy matter to "descend the staircase", and we could eliminate much of the redundancy associated with shotgun sequencing. Unfortunately, this conversion of sequencing labor into mapping labor requires an enormous amount of preparatory work, including overcoming many of the difficulties that presently attend mass sequencing.

A second approach looks to robots to speed up the conventional sequencing process. As a simple matter of mathematics, increasing the rate at which one step of a linear sequential process is carried out does not translate directly into speeding up the entire process. So long as there remain recalcitrant bottlenecks, as in the preparation of the gels and the analysis of sequence data, the power of automation to boost rates will be limited.

Finally, it has been suggested that conventional ideas about tolerable error rates demand too much. Perhaps we could decrease some of the redundancy, aiming for a human sequence with some roughness and inaccuracy, while still deploying the standard algorithms to identify human genes. Taking a clear view of just what would be required to attain the goals described above, it may well be possible to improve rates by lowering the standards on "finished sequence".

Even in combination, these refinements of existing techniques fall short of the hope that the HGP will make sequencing a trivial affair, something from which biologists can liberate themselves. Unless there are large breakthroughs in technology, the suggestion that sequencing the entire human genome is the easiest, or most efficient, way to find all the human genes rings hollow. Nevertheless, the pressure exerted by the HGP may be exactly what is required to force such breakthroughs. The 
task is neither so difficult as to be hopeless, nor a matter of continuing business as usual. Perhaps more emphasis needs to be placed on the improvement of sequencing technology, and it would be wrong to withdraw prematurely from the goal of full sequencing.

It is good to set sights high. Yet, combining an assessment of the value of the sequencing project with concerns about its feasibility, it is reasonable to conclude that settling for less, when less is a set of full sequences for organisms with smaller genomes plus a sampling of the human and mouse genomes aimed at identifying genes by indirect methods, is not settling for much less. At present the best course seems to be to embrace a thorough pragmatism, letting the goals of the HGP evolve in the light both of increasing knowledge of the genomes of model organisms and of the actual development of sequencing technology. With luck, the biologists of a decade hence may be able to commission chunks of sequence as easily as they now order glassware or strains of flies. Alternatively, sequencing may remain an intolerably lengthy process, leaving us with no practical option except to rely on model organisms for insights into how to sample the human genome. The important point is to note that either approach will yield significant scientific information, to prepare for a rich biology of the twenty-first century.

The discussion of this section can be summarized in a number of points.

1. The mapping part of the HGP is already yielding rich returns, and has greatly accelerated the pace of discovery of human genes.

2. The methods of translating sequence data about human beings into biologically valuable knowledge requires comparative studies of model organisms. Without the study of model organisms the sequence of the human genome will be meaningless. 
3. Biologically valuable knowledge about human genes and their functioning will be primarily (possibly entirely) concerned with characteristics that are widely shared among organisms.

4. Sequencing the full human genome may be the most efficient way to identify all the human genes, but this is dependent on breakthroughs in sequencing technology.

5. If breakthroughs in sequencing technology do not occur, there are alternative, indirect, routes to identifying human genes. Although these may not yield quite as complete a catalog as would be obtained from a full sequence, there are reasons to hope that it would provide almost as much biologically useful information. 


\section{The potential and pitfalls of genetic testing}

\subsection{Introduction}

Genetic testing appeared on the medical scene before the HGP began. Even if the project were to cease tomorrow, the technologies of contemporary molecular biology could be -- and almost certainly would be -- used to develop genetic tests of various types. Once researchers know the differences in sequence between those alleles that cause hereditary diseases and those which occur in healthy individuals, it is possible to analyze DNA from a person (obtained from a blood sample or cheek swab) to determine if that person carries one of the unproblematic alleles at a locus or if a disease allele is present. Knowledge of sequence may pave the way for understanding of the causal pathways underlying disease (as described in the last section), and this understanding may allow for interventions to control, prevent, treat, or cure (to be discussed in section 6). But there will typically be a temporal gap, of unknown size, varying from case to case, between knowledge of the sequences associated with healthy and diseased individuals (allowing us to test for the presence of disease genes) and the ability to intervene to help those who suffer from the disease. [NOTE: Here, and throughout this section, discussion will be couched in terms of "genes for diseases". It must not be forgotten that phenotypic traits, in healthy people and unhealthy people, result from interactions between genetic and environmental factors. The dangers of error associated with talk of "genes for disease" will be considered in Section 8.]

When might a genetic test prove beneficial? It will help to start by considering a perfect case. Suppose that someone is informed that he/she carries an allele whose bearers normally suffer from a painful, incurable, disease. Imagine that the test is perfectly decisive: no mistakes are made about the presence or absence of the allele, and, if the allele is present, the disease will invariably be contracted -- unless the bearer is forewarned and makes adjustments in lifestyle. Fortunately, something can be done. Doctors know that if the allele is identified before symptoms occur, changing 
the patient's way of life -- perhaps by taking certain medicines, avoiding certain foods or environmental pollutants, changing everyday habits of work, exercise and leisure -will enable the person to avoid the disease. Making these changes would not be advisable for the general population, for the adjustments to lifestyle are unnecessarily cramping, unpleasant, maybe even damaging to those who do not carry the special allele. Without the genetic test, it is impossible to make rational assignments to courses of treatment, but with the genetic test both people with the allele and people without it have the opportunities to lead healthier lives.

This perfect case is useful because it highlights features that we would like all genetic tests to share. It is helpful to approach the issues surrounding genetic testing by thinking about the ways in which actual tests may fall short of the ideal. The following questions naturally arise:

Do genetic tests deliver reliable information?

Are there useful courses of action that people who are tested may follow, depending on the results they receive?

Assuming that both these questions are answered afirmatively, is it possible to transmit the information in ways that will enable people to make their own, informed, decisions?

These questions concern the role that genetic testing may play in the lives of individuals. Their broader social impact will be considered later (\$\$4.7-4.8). Before taking up the questions in order ( $\$ \S 4.4-4.6$ ), it will be useful first to consider the variety of contexts in which genetic testing might be employed, and some conditions for which genetic tests are already available or likely to become available soon.

\subsection{Contexts of Genetic Testing}

The perfect case, envisaged above, concerned a use of a genetic test to predict the future occurrence of a phenotypic character. Not all uses of genetic tests are 
predictive in this way. Sometimes it is worth employing a genetic test to assist in diagnosing a patient who already shows symptoms. This may occur in the context of initial diagnosis, when a doctor is attempting to find out which disease is causing the patient's symptoms. Alternatively, even after an initial diagnosis has been made, and the patient's condition has been classified, genetic testing may serve in disambiguating diagnosis by revealing particular features of this instance of the disease.

Genetic testing is valuable in initial diagnosis when a person exhibits traits that might be produced in a number of different ways. A forty-five year old woman, who knows she is at risk for Huntington's disease and who imagines that her movements have become clumsy, may wish to know whether she is displaying the first symptoms of Huntington's or whether her condition is due to stress (or some other relatively benign cause). Parents of a child who seems to lack muscle tone may wonder if some form of dystrophy is present. Similarly, genetic testing may be used in disambiguating diagnosis. Some patients who suffer hypercholesterolemia (elevated levels of LDL cholesterol) do so because of features of their diets; others have genetic conditions that result in defective processing of cholesterol by the liver. Genetic testing helps us tell the two groups apart (Khoury et.al. 1993, 84).

Besides these two non-predictive contexts, there are three predictive contexts in which genetic tests might be employed. One, described in the imaginary perfect case, is that of assessing risks for diseases that might develop later in a person's life. A second, familiar to many parents, is the context of prenatal testing: here the aim is to identify genetic conditions in a fetus; amniocentesis (in which amniotic fluid containing fetal cells is removed, usually around sixteen to eighteen weeks gestation), chorion villi sampling (a less well-known technique involving the removal of cells from the fetal part of the placenta, usually after the eighth or ninth week), and the detachment of cells from early embryos (in cases of in vitro fertilization) are all established medical techniques, used as preludes to identifying a growing number of genetic and 
chromosomal conditions. Finally, two people who intend to marry may be tested for the presence of recessive alleles that might be passed on, in devastating combination, to one of their progeny.

A predictive test for Huntington's disease is already available, and it is estimated that tests for certain forms of breast cancer, colon cancer, and Alzheimer's disease may become available in the next few years. Pre-natal testing can already disclose the presence of Down syndrome, Tay-Sachs disease, Fragile X syndrome, and a host of other genetic conditions that, sometimes or often, have profoundly adverse effects on human well-being. Testing couples for the presence of recessive alleles (carrier testing) has been carried out among Ashkenazi Jews, for Tay-Sachs disease, and among Cypriots (and other Mediterranean people), for thalassemia.

\subsection{Examples of conditions susceptible to genetic test}

Before making judgments about the potential and pitfalls of genetic tests it is useful not only to distinguish the contexts of genetic testing but also to consider a range of examples. The following capsule presentations are intended only to single out major features. (For further discussions, see Jones 1988, and Connor and FergusonSmith 1993).

Down syndrome. This syndrome results from the presence of an extra copy of chromosome 21 (or part of chromosome 21). Children with Down syndrome are moderately to severely retarded, and some die young from malformations of the heart. Efforts have been made in the past decades to provide environments that both nurture and challenge children with the syndrome, and this has sometimes produced accomplishments that used to seem impossible. In other cases, however, Down syndrome children do not show significant improvements, even when reared in what are viewed as the most supportive environments. 
Tay-Sachs Disease. This disease appears in infancy, affecting babies who initially seem normal. Progressive degeneration of the nervous system begins during the first year. Most children die before the age of two, and the disease is invariably fatal by the age of four.

Lesch-Nyhan Syndrome. Lesch-Nyhan occurs only in boys (it is due to an Xlinked recessive allele). Boys with the syndrome always suffer mental retardation, and they also experience extreme versions of the pain associated with gout (like gout LeschNyhan involves high levels of uric acid). There is an apparently irresistible urge to self-mutilation: the boys chew their lips and the tips of their fingers until they are raw and bleeding. There are known ways of relieving some of the gouty symptoms, and of preventing the arthritis linked to gout, but none of these methods is effective in treating the neurological and behavioral aspects of the disorder.

Phenylketonuria (PKU). Babies born with PKU (caused by an autosomal recessive) cannot metabolize the amino acid phenylalanine, and, if given a normal diet, have abnormally high levels of phenylalanine and abnormally low levels of tyrosine. In consequence, they suffer severe mental retardation. However, if they are provided a special diet (low in phenylalanine, high in tyrosine), from birth through adolescence, they develop normally. Children who do not carry the recessive allele are harmed by receiving the special diet, and they suffer comparable retardation to untreated PKU children. The special diet is expensive, unpleasant, and requires considerable discipline on the part of children and their families (Nichols 1988 70-72). There is some evidence that PKU children have a more negative image of themselves than do their peers (Moen et.al. 1977).

Fragile X Syndrome. The most common inherited cause of mental retardation is Fragile $\mathrm{X}$ syndrome, a genetic condition in which a gene on the $\mathrm{X}$ chromosome is interrupted by abnormally large numbers of a trinucleotide repeat (CGG). In the first exon of the gene, normal members of the population have between 
6 and 54 copies of this repeat. Those affected with the syndrome typically have more than 300 copies, and may have as many as 1500 . Those with an intermediate number of copies are said to have the premutation: they do not usually display any symptoms, but there is a significant chance that they may transmit to their children a gene with an even larger number of repeats, thus causing the children to have the full syndrome. Women with the full mutation are sometimes mentally handicapped (in about 20-30\% of cases there is moderate retardation), and, in the population so far tested, men are always mentally handicapped to some degree (see the discussion in $\$ 4.5$ ). The genetics of the situation is further complicated by the fact that mitotic division can apparently sometimes produce larger repeats, so that the tissues of those with Fragile $\mathrm{X}$ are usually a mosaic. It is plausible to suppose that the neurological effects vary with the number of the repeats found in neurons, so that some individuals with long repeats might be relatively lucky (the cell divisions giving rise to the neurons do not increase the size of the repeat), while others with shorter repeats might be unlucky (the cell divisions giving rise to the first neural cells all make a dramatic increase in the size of the repeat). Although IQ scores among Fragile $\mathrm{X}$ boys and men range from borderline retarded to severely retarded, almost all male patients suffer from behavipral problems: they frequently engage in perseverative speech, bite their hands, and show some of the emotional responses associated with autistic children (most notably a lack of appropiate responses to the emotions felt by others). Contemporary medical practice can do little to alleviate these problems. Chemical treatments with doses of folic acid, once regarded as promising, are now widely viewed as useless, and, although intensive and personalized programs of support have led to behavioral improvements in a number of instances, many Fragile $\mathrm{X}$ patients are unable to function outside institutionalized settings (for a thorough review of the syndrom, see Hagerman and Silverman 1991).

Myotonic Dystrophy. Like Fragile X syndrome, myotonic dystrophy results from an unusually long trinucleotide repeat (CTG), in this instance within a 
dominant gene. The prognosis for children is quite variable. Children with a single copy of the mutant allele always show some muscular degeneration, but this can begin at any time from birth to early adulthood: some children die at birth, others have little muscle tone from birth on (and also suffer mental retardation), yet others develop normally to adulthood (and themselves have children). The severity and time of onset of the disease seems to be correlated with the length of the trinucleotide repeat.

Huntington's Disease. The recent sequencing of the gene for Huntington's Disease discloses that this type of correlation is no isolated phenomenon. Huntington's disease is caused by the presence of an abnormally long trinucleotide repeat (CAG) in a dominant gene near the tip of chromosome 4: unaffected people have between 11 and 34 repeats, those with Huntington's Disease have more than 42, sometimes as many as 100 . Huntington's Disease is a late-onset disorder, striking both men and women between the ages of thirty and fifty, -- age of onset apparently being correlated with the length of the repeat -- which brings years of ravaging neural degeneration, physically (and, surely, initially, mentally) painful to the sufferer, and emotionally agonizing to those who watch the living death of a person they once knew, admired and loved. As yet, there is no treatment for the disease.

Cystic Fibrosis. This condition is caused by a recessive allele on chromosome 7; it afflicts about one person in 2500 (among people of Northern European origin), with a lower frequency among American Blacks and people of Asian descent. About a decade ago, patients with CF typically died in childhood or early adolescence. Some children develop complications of the pancreas or the intestine, but the most common effect of the disease is on the respiratory system. Clogged airways in the lungs provide a breeding ground for infectious agents. Antibiotics and the use of "chest percussion" (a process in which the child is struck on the chest to loosen the mucus, which is then expelled by coughing) have considerably extended the lifespan, and the median age at death is now 29 , with significant numbers of patients living into 
their forties and fifties. The severity of the disease varies widely. The gene responsible has been mapped, cloned, and sequenced, revealing that a common mutation, found in just under $70 \%$ of carriers, involves a three base deletion in the normal allele. Unfortunately, the remaining cases have proved to be heterogeneous, and over $\mathbf{2 0 0}$ mutations at this locus are now known. Some combinations produce symptoms very like those caused by two copies of the predominant mutant allele, others are highly deleterious, a few combinations are relatively benign. The current prospects for CF patients are uncertain: dramatic improvements have occurred, and more are predicted; in Francis Collins' apt phrase, the condition being tracked is a "moving target" (for further discussion of CF, see (OTA 1992)).

The diseases so far discussed are large effects of changes at a single locus. Once again, it is important to recognize that most phenotypic traits result from interactions among genes and from interactions between genetic and environmental factors. Nonetheless, disorders in an organ or a tissue may be brought about because a mutant allele (or combination of alleles) interferes with the normal causal pathways. Aspects of an organism that are brought about in a complex fashion may be disrupted in a wide variety of ways, so that what we pick out as "the same disease" may have quite different causes in different individuals. These points will be discussed in much greater detail in section 8 . They are illustrated by several types of disease that are much more common than those considered above.

Cancer - a very brief overview. Cancers occur because of misregulation of the mechanisms for cell division, so that a cell line goes into overdrive, producing an abnormally large number of descendant cells. Misregulation is usually caused by the activation of some genes and the loss (or modification) of others. Typically, many changes are required, and cancer only occurs after "multiple hits". During the course of a human lifetime, there are many mitotic divisions, and, at each division, there is a small chance that some crucial piece of DNA will be altered. Environmental agents 
can trigger these changes, or they can activate a gene that would not normally be switched on. Genetic susceptibilities can also play important roles. Some people may carry alleles that dispose their cells to tolerate copying errors in DNA replication -perhaps if those cells are exposed to particular environmental agents, perhaps in a wide variety of environments. Others may start missing a piece of DNA whose inactivation is one important step in the formation of a tumor: if (say) six modifications of the DNA are needed for the tumor, these unfortunate people have already taken the first step at conception, and only need five further "hits". Cancers can thus occur in some people because of the action of environmental agents -- as when people are exposed to radiation or to mutagens which greatly increase the probabilities that their mitotic divisions will go awry. "The same" cancers occur in others because they bear alleles at several loci which collectively permit inexact copying at some crucial area of the genome. Yet others contract cancer because they carry, from conception, a nonfunctional tumor suppressor gene or genes whose protein products can activate an oncogene (a tumor promoting gene). To simplify a complex story, cancers typically result from complicated interactions of genes and environmental factors, with different mixes of those factors in different cases.

Colon cancer. Genes implicated in many instances of colon cancer have now been cloned and sequenced. These genes play an important role in ensuring the fidelity of DNA replication, and people who have mutant alleles are predisposed to tolerate copying errors in mitotic divisions. Tests for identifying mutations at the pertinent loci are being developed, and these tests are expected to pick out those people who are at risk. Medical researchers hope both that the knowledge of the sequences will lead to methods for intervening to prevent the growth of malignant tumors, and that those at risk can be monitored so that growths in the colon can be detected at early stages and full-blown colon cancer prevented. 
Breast cancer. Several groups of researchers are currently attempting to clone and sequence a gene that predisposes a small percentage of women to early-onset breast cancer (and, with lesser frequency, to ovarian cancer). In the families investigated so far, the risks of contracting breast cancer, at any age, for women who carry the allele are significantly above those for the general population. Again, it is hoped that the identification of the gene will enhance our understanding of the causal pathways involved in many forms of breast cancer, and that, in advance of the development of strategies for intervening, a genetic test will be able to identify those women at high risk, who may then be monitored more closely (see M-C. King et.al. 1993).

Hypercholesterolemia. Elevated levels of LDL cholesterol (so-called "bad" cholesterol) play a role in some cases of heart disease. Failure to break down molecules of LDL cholesterol leads to the buildup of cholesterol in the arteries, sometimes causing clogging that culminates in strokes and heart attacks. The liver carries out the function of breaking down cholesterol molecules, and people who carry a mutant LDL receptor gene have livers that do not perform the job efficiently, suffering hypercholesterolemia in consequence. Only about $10 \%$ of those affected with hypercholesterolemia carry a mutant LDL receptor allele, and, although excess LDL cholesterol increases the chance of heart attack or stroke, many people with high levels of LDL cholesterol do not have cardiac problems (Holtzman 1989 23, 44). Here, as in the cases of the cancers, genetic testing is inevitably bound up with probabilities.

\subsection{Reliable Information?}

There are three potential sources of worry about the reliability of genetic testing, two of which can be relatively swiftly treated. Are the genetic tests that will be available likely to yield significant numbers of false positives (instances in which a 
person who does not have the genetic condition is identified as having it) or false negatives (instances in which a person who has the condition is identified as not having it)? Are genetic tests vulnerable to laboratory errors that will yield misleading results? There is a deeper, and more complex, question about the reliability of the information to be obtained from genetic testing. In some, though not all, contexts, patients and doctors need to be able to draw conclusions about phenotypes, and even though genetic tests are wonderfully accurate and informative about genotypes, they will not discharge some of their functions if there is no reliable way to generate predictions of phenotypes. So the question that will occupy most of this section is: Will genetic tests serve to make reliable phenotypic predictions?

False positives and false negatives. Most genetic testing carried out during the 1980s was restricted to deciding whether an individual, from a family in which a disease allele had been identified, carried the disease allele in question. The strategy was to find markers (usually RFLPs -- see section 2) regularly transmitted with the allele, and assess the individual for the presence of the marker. Testing using RFLPs has some well-known difficulties: there can be occasional recombination between the marker and the disease locus, producing both false positives and false negatives (in most cases these are very low, of the order of a few percent). In addition, carrying out the test requires other members of the family to provide information (and this has occasionally proved problematic); the method is also limited to use on families in which hereditary disease can be tracked using classical genetic methods, and it cannot be used to assess risks for members of the general population.

Sequencing changes the picture. Once the DNA sequences of normal alleles at a locus and the sequences of mutant alleles that give rise to disease are known, then it is possible to determine unambiguously whether an individual carries one of these alleles. False positives and false negatives no longer arise from recombination 
between marker and locus. Moreover, samples from other family members are no longer required, and the testing can be carried out on the general population.

Laboratory errors. It is a familiar fact that laboratory procedures can go wrong, and that laboratory technicians can make mistakes. Quality control in genetic testing is as important as in other types of medical testing, but there is no reason to think that genetic testing is especially vulnerable to human errors that will produce misleading reports. The reactions involved in sequencing (and in RFLP testing) are sufficiently well understood to be carried out with great accuracy, and though human errors do occur at a low rate, the vast majority of them produce results that wear their own misinformativeness on their faces (gels are found to be blank, bands on gels are smeared, the control lanes do not have the expected features). Probably the most worrying type of error is likely to come from the confusion of samples -- through mislabelling or misrecording -- and such potential mistakes threaten an enormous number of medical (and other) practices.

Phenotypic Predictions. As already noted, the most complex issues concern phenotypic prediction, especially when it is suggested that the HGP will pave the way for us to receive detailed information about our risks for all kinds of diseases, a socalled "genetic report card". There are two potential sources of trouble. First, and most important, is "variation in penetrance": differences in the degree to which people who carry a particular allele are afflicted with a disease. Second is heterogeneity of the causal basis of a disease. There are examples in which a large number of geneenvironment combinations can cause disease, and if the cases are sufficiently various there may be no basis for reliable predictions: effectively there are so many combinations, some producing disease and others not, that many of the cases that arise are either unprecedented, or else similar to so few known instances that prediction is uncertain (Holtzman 1989, Andrews et.al. 1994, Nelkin and Tancredi 1989). 
Employment of genetic tests in the non-predictive contexts -- initial diagnosis and disambiguating diagnosis - is unaffected by these complications. When a patient presents with symptoms, the task is not to predict the occurrence of a disease but to analyze the causes of the symptoms. Genetic testing would only be confounded in such cases if identification of an allele left open two (or more) possible diseases that might manifest the observed symptoms. The sources of unclarity stemming from variation in penetrance and causal heterogeneity do not generate trouble in diagnostic contexts because relevant facts about the patient's phenotype are already known.

Predictive contexts -- evaluating risks of later disease, testing a fetus in utero, and assessing whether prospective parents carry dangerous alleles -- are much more problematic. Here the goal of the test is ultimately to inform people about a future phenotype, or, more frequently, about the chances of having a future phenotype. Difficulties arise because the relevant probabilities are unknown or are easily confused with quite different probabilities that are known.

As several critics of genetic testing (most notably Jonathan Beckwith) have pointed out, we can easily be seduced into fallacious reasoning because we ignore the ways in which the bearers of a particular genetic condition have been identified (Beckwith and King 1974, Beckwith 1993, Hubbard and Wald 1993). An especially powerful illustration comes from the recent history of behavior genetics. When it was found that prisons and mental institutions contained more men with an extra $Y$ chromosome than had initially been expected, the immediate conclusion was that the XYY karyotype (the chromosome type of males with an extra Y chromosome) was at least an indicator (and, quite probably, a cause) of criminal tendencies and/or mental disorders. To reach that conclusion is to propose that the probability that an XYY male will commit a crime or be mentally disturbed is high. Now the direct way of discovering that probability is to look at the general population and find the frequency with which XYY males go either to jails or to mental institutions. To look at the 
prison population alone and find a number of XYY males does not license even preliminary conclusions about the pertinent probability unless there is some background knowledge about the frequency with which XYY males occur in the non-prison population. Subsequent investigations have underscored this point about the mathematics of probability, by revealing that $96 \%$ of XYY males lead normally peaceful lives, never having problems with the police or suffering mental disturbances. The idea that XYY is a sign of criminality has been effectively refuted.

Standard methods in human genetics proceed analogously to the initial detection of XYY males. Genes are discovered through the comparison of afflicted and unafflicted members of families in which the disease occurs. Within these families the frequencies with which bearers of the allele have the disease are typically high (often 1 or close to 1), precisely because families in which there is so clear a correlation are most revealing for purposes of genetic analysis. The problem is now to compute the frequency with which members of the general population who carry the allele have the disease. The solution would be easy if the sample of people in the data accurately represented the general population. However, as noted, the sample is biased by choosing families within which the genotype-phenotype correlation is very tight -perhaps because of special envirnmental features, perhaps because of aspects of the background genotypes. So simply projecting the frequencies from the data would be a mistake.

At present, the extent to which various genetic conditions obtain in the general population is uncertain, and the extent of the uncertainty varies from case to case. Given background knowledge about Tay-Sachs and Huntington's disease, it is very hard to dispute the conclusion that bearers of the pertinent alleles will invariably develop the terrible condition. At the opposite extreme are the various cancers, for which statistics on occurrence are drawn from the family studies, and may not represent the general population at all. The wider the variety of families sampled, the more 
likely it is that bias within the sample will be reduced -- but so long as tests are carried out only within families with histories of cancer, some guesswork will be involved in using the presence of an allele in a member of the general population to predict the risk of cancer. The guesswork could be eliminated by doing what was done for the XYY karyotype, to wit measuring directly the frequency with which members of the general population develop the disease.

Fragile X syndrome provides an intermediate case. Nobody has yet investigated to see whether or not the general population contains large numbers of people with the long CGG repeats associated with the disease alleles. If a couple is told that the male fetus carried by the woman bears the $\mathrm{Fra}(\mathrm{X})$ gene, the only estimate of the probability that the fetus would develop into a child with the syndrome is that obtained from families in which that gene has been identified. Initially, the families used to pick out Fra(X) were those that showed a tight association between nearby markers and the disease phenotype. Once the $\operatorname{Fra}(\mathrm{X})$ gene was discovered and sequenced, boys with the typical symptoms were tested, and some were found to carry Fra(X). The pattern of transmission within their families can be - and has been -investigated without presupposing that male $\mathrm{Fra}(\mathrm{X})$ bearers always suffer mental retardation (and the other symptoms). The fact that in these families no males have yet been found carrying $\operatorname{Fra}(\mathrm{X})$ who are phenotypically normal buttresses the case for thinking that the probability that a boy with $\mathrm{Fra}(\mathrm{X})$ will suffer some degree of mental retardation is very high. Yet, when we consider the importance of the decision that the couple is trying to make through genetic testing, it seems irresponsible to calculate the probabilities in roundabout ways: why not directly sample the population?

It might be thought that this direct approach is impractical, costly, or invasive. However, there seem to be no obvious difficulties in obtaining blood samples or cheek swabs from a sufficiently large number of children or adults. To reduce worries about misuse of genetic information and invasion of privacy, these samples could be made 
completely anonymous, each marked only with a notation about the presence or absence of the phenotypic condition under study. Some simple calculations show that sampling 10,000 asymptomatic males, and discovering that none of them carried $\operatorname{Fra}(X)$, would show that the probability that a male bearing $\operatorname{Fra}(X)$ will have Fragile $X$ syndrome is greater than $5 / 6$. (Technical Note 1 , at the end of this section, discusses the probabilistic issues in more detail and gives an explanation of this result.) Plainly, testing 10,000 people has costs, but in such broad population testing many of the ancillary expenses (such as counselling -- see below) can be avoided, and the project could be carried out at a cost on the order of $\$ 1$ million. Although this may appear excessive, we may think about it as a necessary overhead to the other costs of finding and sequencing the gene, and developing and refining a genetic test, an expense that we need to bear if genetic tests are to provide reliable information that can aid people in making responsible decisions.

In the case of Fragile $\mathrm{X}$ syndrome, the genetic heterogeneity associated with the number of CGG repeats would make a more fine-grained statistical analysis highly desirable: ideally, we would like to know the frequencies, in the general population, with which men with various lengths of repeats have various phenotypic conditions (ranging from those who are normal to extremely severe instances of Fragile $\mathrm{X}$ syndrome; Fragile X Syndrome is already known to be variable in women). Cystic fibrosis is just as complicated, and, in this instance, we know in advance that, even in those who bear the allele, there is pronounced variation in phenotype. Most informative would be a detailed statistical analysis exploring the probabilities with which a range of phenotypes occur in individuals with a range of genotypes who encounter a range of environmentally relevant factors (perhaps quality of early nutrition, age at diagnosis, frequency of use of various antibiotics). In principle, an analysis of this type could help not only in providing relevant information in the 
context of genetic testing but also help in tracking down ways of ameliorating the condition of those with $\mathrm{CF}$.

In the contexts of pre-natal testing and prediction of disease risks, genetic testing will be most informative if the probabilities do not simply reflect what happens across a heterogeneous mixture of environments. Suppose that people who have the gene in "bad" environments manifest the disease, while those who have the gene in "good" environments have a much milder version, or are even completely symptomfree. If "bad" environments predominate, the probability that bearers of the gene will have the disease will be high. Yet what is relevant for the prospective parents or the person at risk is the possibility of creating a "good" environment. When we know how to differentiate environments, and how to bring those environments about, we need not stand passively by, accepting the "average" risks as given. In many instances, we are ignorant and powerless; in others we are not. These kinds of cases need to be treated differently: in the first, constructing an "average" risk is the best we can do; in the second, responsible decision-making requires knowledge of the probabilities of the phenotype against a partition of the environment.

The case of Down syndrome has been widely used by disability activists and opponents of the HGP to cast doubt on the value of genetic testing (indeed to insist on the harm of genetic testing). There is no doubt that dedicated parents, siblings, social workers and doctors have effected a large transformation in the lives of Down syndrome children. It would be quite wrong for parents to be told that the probability that a child with an extra piece of chromosome 21 will be able to lead a life with some degree of self-sufficiency and personal satisfaction is low, when this probability is based on averaging across cases in which children are provided nurture and support and those in which they are not. What prospective parents need to know is the full range of phenotypes associated with the chromosomal condition, and the probabilities associated with each of these in situations where great support is given (environments 
with the greatest level of support available to them). Warning against assuming the worst, or assuming the average is salutary -- but equally there is a danger of overromanticism, of assuming the best. Anecdotal data about the lives of children with Down syndrome are available, but a more systematic survey of the phenotypes associated with favorable environments is urgently needed.

Assembling the relevant statistics for Down syndrome, Fragile $\mathrm{X}$, or CF is a large task. When we envisage the full range of genetic tests that the HGP promises, the challenge is truly extraordinary. Within a decade it will very likely be possible to give a sample of blood to a doctor and receive a "genetic report card": that card will identify those loci at which the donor has unusual alleles. Yet if the card is to provide reliable information that can be used in making decisions, there must be a rich background of information about the probabilities of phenotypes given particular genotypes. Those probabilities should be based on surveys of the general population that differentiate environments to the extent to which it is useful to do so. Assembling this informational context for genetic testing, for the full range of conditions, is so Herculean a venture, that any useful version of the "genetic report card" seems utopian. The HGP will greatly increase the number of potential genetic tests, but it will leave us with the problem of choosing which of these tests are most urgently needed. Given the work, and expense, involved, it will be necessary to make priorities about which kinds of conditions most urgently require testing, and to ensure that reliable statistics are available for these.

\subsection{Nothing to be done?}

Courses of action, based on information from a genetic test, plainly vary with the context in which testing is carried out. Diagnostic genetic testing might help doctors in finding treatment, or enable them to avoid courses of treatment that are useless or even deleterious. Similarly, in disambiguating diagnosis, patients will be 
aided if treatments vary in their efficacy or in their side-effects, depending on the causal basis of the disease condition. Predictive testing for diseases can help if steps can be taken to prevent the disease, reduce the risk, delay the time of onset, or reduce the severity. Pre-natal testing might either prepare parents for the birth of a child with a condition that requires special care or intervention, or lead them to terminate pregnancies which, if continued, would issue in children destined for lives of suffering or of severely confined opportunities. Finally, in cases of carrier testing, people may either be freed from the anxieties that hang over their plans for a family or, if given bad news, may choose to have children only with a commitment to pre-natal testing (or through adoption).

In the last two contexts, there is plainly something that can be done (although the use of genetic tests in deciding whether to continue a pregnancy raises both general questions about abortion and more special issues about the threat of eugenics: see section 8). However, in each of the other contexts, there may be no course of action beneficial to the patient which the test makes possible. This may happen for either of two different reasons: even in the light of the genetic knowledge, nothing could be done to prevent, treat, or cure; or, the same interventions would be carried out whether or not we knew the patient's genetic condition. PKU provides a relatively pure case in which the genetic knowledge is absolutely crucial, for here everything depends on matching the diet to the child's genetic condition. Huntington's disease is a clear example of a case in which (at least at present) nothing can be done to reduce the risk, delay onset or lessen severity. In other instances, especially some genetic conditions that affect risks for cancer and heart conditions, advice can be given -- patients can be told to exercise, not to smoke, and to avoid fatty foods - but these pieces of advice would have been available, and have been good, whether or not the genetic test had been carried out (Hubbard and Wald 1993 80). (One way of thinking about the future of genetic testing would be to take the context of disambiguating diagnosis as a 
paradigm, starting not with diseases but with strategies for promoting health, and asking if there are genetic bases for exceptions to the blanket advice we give. It would be worth knowing, for example, whether some people have a genetic predisposition not to thrive on a low-fat diet. That knowledge would not only aid people with the predisposition, but also reinforce the recommendations for the general population.)

Many of the genetic tests already available (or likely to become available) will primarily be employed in diagnostic contexts or in the context of pre-natal testing: Down Syndrome, Tay-Sachs, Myotonic Dystrophy, Fragile X, Lesch-Nyhan, and CF all fall into this category. The principal concerns about these tests center around issues of reliability discussed in the last section. By contrast, tests for Huntington's Disease, for various cancers, and for conditions relating to heart disease (such as hypercholesterolemia) are principally aimed at predicting risks of later disease. Here, in addition to questions about reliability, we need to consider if the knowledge of risks can be translated into useful action.

Knowledge might be valuable in either of two ways: as already noted, it might facilitate some modification of the patient's life that would ameliorate the disease (reducing risk, delaying onset, lessening severity)-- medical benefit; or it might enable the patient to have a better life, even though the course of the disease was unaffected -non-medical benefit. Focusing, for the moment, on the first perspective, potential medical benefit, we can recognize that the test for PKU is wonderfully valuable on this score, the test for Huntington's absolutely useless. Other cases are more complex. Some genetic tests may prompt people who are at higher risk for a disease to undergo monitoring that will enable the disease to be detected early with beneficial results: certain envisaged tests for cancer are alleged to have this virtue. Here we must ask a series of questions:

(A) Would the monitoring be valuable for people whether or not they have the condition detected by the test? 
(B) Does the monitoring succeed in identifying the disease at an early stage?

(C) Does identifying the disease at an early stage improve the patient's prospects?

If the answer to (A) is "Yes", if the answer to (B) is "No", or if the answer to (C) is "No", then the genetic test brings no medical benefit. Conversely, genetic tests bring medical benefits just in case the answer to (A) is "No", and the answers to (B) and (C) are both "Yes". There are grounds for hoping that the tests for alleles yielding predispositions to colon cancer will meet these conditions. Routine inspections of the colon through colonoscopy are both painful and costly, and thus inappropriate for everyone in the population. These monitoring procedures can detect growths at an early stage, and removal of those growths prevents the development of full-blown cancer. If these points are sustained, then a genetic test for susceptibility to colon cancer would be a valuable predictive tool.

Compare this with the envisaged test for the allele implicated in early-onset breast and ovarian cancer. What exactly is a woman who tests positive for this allele to do? The first thought is that she should perform self-examinations and have mammograms at earlier ages than women who lack the allele. Breast cancer is sufficiently common, and the consequences sufficiently grave, for self-examination to be recommended to all women, whether or not they carry the allele. Setting on one side the debate about mammogram safety, there are significant limits to the ability of mammograms to detect small tumors, especially in younger women: as women approach, and pass through, menopause their breast tissues become less dense and more fatty, so that mammograms offer clearer pictures of potentially dangerous changes. Sadly, for virtually all women in their twenties and thirties, mammograms are of very little help in the early detection of tumors. Finally, it is far from clear that early detection of the cancers associated with the breast cancer allele will enable a significant improvement in treatment. While uncovery of the allele may well ultimately lead to 
an improved understanding of breast cancer, serious thought needs to be given to the interim value of a test.

Even without the genetic tests, it might be worth recommending mammograms for those women whose breast tissues allow the detection of changes -- assuming that early detection, in general, increases chances of improving the consequences.

Moreover, genetic tests might serve a useful function in those families in which the incidence of breast and ovarian cancer is very high (M-C King et.al. 1993) -freeing some young women from a shadow that hangs over them, perhaps prompting others to the radical option of bilateral mastectomy (although, tragically, there are known instances in which this has not proven effective).

In general, the potential medical benefit of a genetic test should be carefully assessed by answering (A)-(C). If the answers support the conclusion that the test can bring medical benefit, then more detailed questions about the size of that benefit, and the costs of testing, can be posed. By how much does monitoring increase the chances of early detection? By how much does early detection improve the patient's prospects? What are the negative consequences of monitoring?

Even when genetic tests bring no medical benefit, they may still prove worthwhile because of the non-medical benefits they bring. Greater knowledge can bring greater autonomy. Well-informed agents may recognize that even bad news is welcome, giving them opportunities to fashion, within cruel constraints, futures that best achieve what is important to them.

Since the arrival of testing for Huntington's disease, it has become apparent that many fewer people, confronted with the actual opportunity to know their status, have volunteered for testing than was predictable in advance, given those people's anticipations of what they would do (Wexler 1992, Andrews et.al. 1994 88-89). It is easy to draw on everyday experience of human character to recognize that knowledge will be valuable for some, while ignorance will be better for others. One 
person, learning that Huntington's disease will strike within two decades might undertake a rational reorganization of her life, making sure that she completed the projects that were most important to her. Another might be so crushed by the news that she would overreact, abandoning plans and long-cherished ambitions that are, in fact, still attainable (see (Holtzman 1989 180) for reports that, in one study, thirteen of eighty-seven people willing to be tested for Huntington's disease declared that they would commit suicide if the results showed that they had the disease).

Section 4.4 emphasized the need for genetic tests to be embedded in a rich informational context. Such tests also need to be placed in a system of social support. If genetic tests are genuinely to increase the autonomy of those tested, it is important to ensure that the simple ethos of looking truth in the face is not unthinkingly applied. Potential test-takers must be encouraged to decide if their well-being will indeed be enhanced through taking the test, and because of human proclivities for self-deception, many of us will need help in making that decision.

Enhancing the autonomy of individual decisions requires both controlling those pressures that would coerce people into taking tests and providing positive support for their efforts to work out if the test will benefit them. On the first score, there is a real danger that tests will be institutionalized in medical practice, that they will become automatically recommended (perhaps by doctors who fear suit if they do not encourage their patients to take the tests), and that, in some instances, any medical benefits obtained may be outweighed by the non-medical harms caused to the person tested. As several critics have pointed out, tests are often developed by people who have an interest in the fortunes of a particular biotechnology company, and there will doubtless be market pressures for the use of tests, whether or not they benefit the public (see Hubbard and Wald 1993, Nelkin and Tancredi 1989). Attending to these dangers requires ensuring, especially for those tests in which no medical benefit is expected, that the potential subject of the test fully understands the option of declining to be 
tested. On the second, more positive, score, it will be necessary to develop a far more detailed (and effective) practice of pre-test consultations, involving genetic counsellors who are sensitive to the possibility that tests may cause some people serious psychological damage. Although the problems are already with us, the proliferation of genetic tests from the HGP will make it imperative to address them.

The principal conclusions of the present discussion are: (i) concerns about the value of genetic tests arise primarily in the contexts of prediction of later disease, and, sometimes in analogous fashion, in contexts of diagnosis; (ii) to assess the medical benefits of a test it is necessary to ask whether the information to be derived will make a difference to the reduction of risk, the delay of onset, or the lessening of severity, and the inquiry can be focused by posing questions (A)-(C); (iii) to assess the nonmedical benefits of a test, it is crucial to take into account individual psychological variation, to avoid institutions that coerce people into taking tests and to promote pretest counselling.

\subsection{Problems of Communication?}

Thinking about genetic testing in the abstract is likely to conjure up the image of an ideally rational agent, fully self-aware, who can be trusted to make optimal use of the information presented. Recognizing human psychological variation is only to appreciate one dimension along which actual practice differs from the ideal. It is easy to fall into the trap of stereotypical thinking: the person who comes for genetic testing is perceived as affluent, with resources that will suffice to translate the genetic information and the doctor's recommendations into responsible action, someone for whom the uncertainty at which the genetic test is directed is the focal problem in an otherwise serene life. People with these characteristics may currently be overrepresented in genetic counselling situations, but they are not representative of the population, even in an affluent society like the US. If genetic tests are to serve the 
needs of all citizens, it would be wrong to evaluate them from a perspective only enjoyed by the most privileged (Duster 1990 64-6).

Members of ethnic groups most at risk for stigmatization (see section 5), and other adverse effects, through the distribution of genetic information are also the people least likely to benefit from genetic testing. Their access to the medical system is more restricted, and their ability to make use of genetic information is more limited. Furthermore, people from many strata in US society, not just those from the poorest and least privileged segments, may find it difficult to assimilate the information provided by genetic tests. For all the efforts of genetic counsellors, clear communication of the possibilities of various options simply does not occur (Katz Rothman 1986, Rapp 1994, P. King 1992).

A genetic counselling session may reasonably be counted as problematic when, at the end of the session, either the counsellor believes that the most important points have not been understood or the person counselled believes that the most important concerns have not been addressed. According to research on current counselling practices, counsellors are only aware of what their clients want to discuss in $42 \%$ of cases, and the data on communication of risks paint an extremely dismal picture (Holtzman 1989 174-178). Perhaps standards are too high or judgments are too pessimistic, but it seems plain that rethinking and revision of counselling practice is in order. The conclusion of $\$ 4.5$ that counselling is needed to help people decide whether a genetic test will bring them nonmedical benefit cannot be sustained unless changes in practice support the idea that counselling can increase individuals' ability to make autonomous decisions.

Conveying detailed information about genetics is evidently difficult. Yet what those counselled primarily need to know are the following: their risks of acquiring a phenotypic condition, given the environments they are likely to inhabit; the nature of that condition; how the risks of the phenotype would be affected -- if at all -- by 
manageable changes of lifestyle or other interventions; and, in accordance with $\$ 4.5$, whether they are people who could reap non-medical benefits from information about their risks. It seems likely that those trained in genetic analysis are not necessarily the best people to transmit these types of information, and that the counselling framework that surrounds genetic testing might well employ counsellors with different kinds of expertise.

Currently, the official ideology of genetic counselling urges "nondirectiveness", the eminently reasonable thought being that counsellors should not impose their own values on those they counsel, but leave those counselled to make decisions that are genuinely their own. Although this may be a splendid ideal, the transition to a society in which genetic testing is far more widespread than at present will almost certainly multiply the number of situations in which "respecting the autonomy of the counselled" would be a bitter joke. Counsellors will surely have to learn when they should restrict their contributions to the presentation of factual information, and when they are, in effect, the last and only possibility for articulating a course of action that will genuinely meet the needs of agents whose awareness of their own predicaments and options is quite limited.

Providing a system of support that will enable important points to be communicated, and that will enhance the decision-making -- and the lives -- of people who do not fit the stereotype of the client in the ideal counselling situation is difficult. But it is not impossible. Nancy Wexler's sustained efforts among the Venezuelans of Lake Maracaibo -- a population whose members are at risk for Huntington's disease -shows clearly how ignorance can be overcome, and how molecular medicine can be made to work for those who are poor and underprivileged (Wexler 1992). To do this on the scale of an urbanized society will take a considerable economic and social commitment - it will require substantial investment in background counselling, research into the problems that attend current counselling practice, and imaginative 
solutions to them. When genetic testing is envisaged on the scale of "genetic report cards", such investment is almost certainly impossible. When it is conceived in a more limited way, in terms of diagnostic testing by doctors and predictive testing for those diseases and pre-natal conditions that are given highest priority, there is surely a scale on which genetic testing can be successfully managed. It is imperative to remain alert to the danger that, without any attention to the necessary background, both statistical ( $\$ 4.4)$ and social $(\$ \$ 4.5,4.6)$, genetic testing will cause both good and substantial harm, and that, because of background inequalities within US society, the harm will fall disproportionately on those whose lives are already hardest.

\subsection{Forbidding, requiring, and encouraging genetic tests}

The guiding assumption of the discussion so far has been that people should be free to take tests that they regard as valuable. It has been supposed, in effect, that the HGP will help create a huge supermarket in which our descendants can shop for genetic tests, and the main questions have focused on the background conditions needed to make the wares worthwhile. The tests supplied would just be those that the public demanded: nothing would be forbidden, nothing required, nothing encouraged.

This perspective flows naturally from principles of individual liberty. We might begin with the idea that nobody can be compelled to take a genetic test, however beneficial it might be for the person to do so. By the same token, nobody can be prevented from taking a genetic test, no matter how frivolous, even harmful, taking that test might be. Unless, there is reason to think that the person is incompetent to make the decision, it would be wrong to intervene. Moreover, making a decision that is against one's best interests -- or against others' perceptions of those interests -- is not automatically a sign of incompetence.

However, there are limits to individual liberty. When our decisions have consequences for the well-being of others, it is quite appropriate to consider the welfare 
of those who would be affected and third parties sometimes have the right to intervene. Can such situations arise with respect to genetic testing? Consider four hypothetical scenarios, some of which are clearly real possibilities:

(a) a potential subject for a test to determine whether the allele for a lateonset disease is present has young children who will be affected if the disease strikes: should the person be required to take the test?

(b) a child is born to parents who refuse to grant permission for PKU testing: should such testing be carried out anyway?

(c) a person is at risk for a treatable condition with a genetic basis, which if left untreated makes him a public health hazard (perhaps because his lungs provide infectious agents with an opportunity for evolving drug-resistant strains): should the person be required to be tested and to undergo treatment if the test proves positive?

(d) the mother of a fetus at risk for an inevitably fatal and painful early degenerative disease (such as Tay-Sachs) or for a severely handicapping syndrome (Fragile $\mathrm{X}$ ) does not wish to have prenatal testing: should she be required to do so?

No doubt it would be reasonable to try to persuade the parent in (a) to act in the children's best interests. Yet, if the parent remains adamant, to intervene would seem an unwarranted interference with liberty. The US, like other societies, acknowledges the rights of parents to engage in risky behavior. If sad consequences do ensue, then the state must do what it can to alleviate the suffering of the innocents. It is important, in this case, that something can be done.

Sometimes, however, the harms inflicted cannot be avoided once the original action has been taken. In (b) there might be irreversible bad consequences for the child. Similarly, in (c), the agent's decision to refuse testing or treatment could bring disease or death to many others. - Just as the state has the right to compel citizens not 
to light backyard fires under conditions of extreme drought, so too it would have the right to demand that the imaginary subject in (c) take the genetic test.

If this is correct, then there are conditions under which the consequences for others are sufficiently severe to override someone's right to refuse a test (either for the person himself or for someone with regard to whom the person usually has the right to make decisions, such as a minor child). How far does this qualification of individual rights to free decisions about tests extend? Might a similar justification apply to require pre-natal testing and termination of pregnancies which would issue in the birth of a severely deformed child whose life would be inevitably brief and excruciatingly painful? Historically, steps in this direction have led further, and contemporary critics of genetic testing worry that we may pass quickly to full-blown eugenics.

The broader issues involved here will be taken up in section 8 . For the moment, discussion will be restricted to the question of whether the proposed answers for (a), (b), and (c) demand restrictions of individual liberty in case (d). It is helpful to make explicit the principle that appears to lie behind the legitimacy of intervention in (b): the restriction of parental freedom appears to be a lesser harm than the causing of irreversible damage to the child. In similar fashion, we could require pre-natal testing and treatment of the fetus if that treatment would prevent the same kind of tremendous damage, provided that the woman also had the option of terminating the pregnancy. Imagine that molecular genetics makes it possible to test for the presence of the LeschNyhan allele prior to the point in development at which the allele plays its most important role, and that interventions at that point would prevent the syndrome, allowing normal development. Suppose that a woman is at significant risk for bearing a child with Lesch-Nyhan syndrome, and that she wishes both to continue the pregnancy to term and not to have the test (and consequent treatment for the fetus). It is possible to acknowledge her right to reproductive decision, while seeing the decision to continue the pregnancy as involving a commitment to avoid irreversible damage to 
the child that the fetus will become. (At this point it is extremely important to emphasize a point made eloquently by many writers: almost all women who act in ways that risk harm to a fetus do not wish to cause such harm; interventions to help the fetus should properly be viewed as ways of alleviating the pressures in extraordinarily difficult situations; the hypothetical case is intended only to draw out the abstract implications of the principle used in (b), not to represent a situation of conflict between woman and fetus, which is, almost always, an artefact of discussions of social issues. See, for example, Katz Rothman 1986, Cowan 1992).

The hypothetical case makes it evident that the line of reasoning used in (b) and (c) does not extend to mandatory prenatal testing for conditions about which nothing can be done except to terminate the pregnancy. In the real case of PKU, and the imaginary, analogous case involving pre-natal testing and treatment for Lesch-Nyhan, there is an identifiable individual to whom irreversible damage would be done in the absence of active intervention. If the test is taken and the treatment administered, there is an identifiable individual whose life is enormously improved. In cases where the only option is to terminate the pregnancy, there is no individual whose lot becomes better. Possibly, by intervening, we would make the world better than it would otherwise have been -- for a world in which the Tay-Sachs bearer dies in utero may be a world with less suffering than one in which the degenerative disease takes its course - but no one has been benefited by doing so, nobody has been appropriately protected from severe harm. The principle used in (b) requires testing and intervention on the basis of a commitment to an individual who would be harmed by not intervening. Prenatal testing in cases in which nothing can be done except to abort affected fetuses does not fall within the scope of this principle. So it is perfectly consistent, and reasonable, to support mandatory PKU testing for newborn infants, and to resist the idea of compulsory prenatal testing for irremediable birth defects. (Of course, there is a line of reasoning, as old as eugenics, which urges mandatory testing on the grounds 
that, without it, the costs of supporting people with debilitating genetic conditions will become too great for society to bear. This argument will be considered in section 8 . According to (OTA 1992) 28-9, almost ten percent of Americans surveyed in 1990 believed that there should be laws requiring abortion when a fetus is found to have "serious genetic defects").

Exactly parallel considerations apply in connection with forbidding tests. Despite the wide range of opinions about when abortion is permitted, very many people would probably agree that decisions to terminate a pregnancy because of the presence of certain kinds of genetic conditions would be morally suspect. Couples who tested their fetuses for the presence of blue eyes, and who decided to abort otherwise healthy fetuses in all cases in which an alternative genotype was present, would have a distorted conception of value. Nonetheless, freedom of reproductive choice can be defended so long as one does not think of the fetus as having rights and interests which it is the state's duty to protect. Ronald Dworkin has argued persuasively that our thinking about abortion should reject the idea that fetuses are persons, and his discussion allows us to honor women's rights to make reproductive choices while simultaneously seeing the termination of pregnancies in some circumstances as "an insult to the value of human life" (Dworkin 1993).

Although there may not be compelling reasons to forbid the usage of certain types of genetic tests, the account developed earlier in this section emphasizes that considerable resources are required for programs of genetic testing and that choices will have to be made. Quite evidently, it would be bad if the genetic tests that were widely available within the US were directed at relatively frivolous conditions ("having blue eyes") while acutely painful or limiting conditions (Lesch-Nyhan syndrome, Fragile X syndrome) went untested. There are serious questions about what kinds of tests are encouraged in a situation in which we can envisage more tests than can properly be supported. How should priorities be set? 
There are two obvious answers to this question. One is to suggest a free market in medical testing, allowing supply and demand to determine which tests are developed and which receive the informational and social backing necessary to make the tests work to the genuine benefit of those tested. The danger with this approach is that any such market is likely to reflect the priorities and values of the most powerful groups within society, and that the interests of others may simply be ignored (Katz Rothman 1986, Hubbard and Wald 1993, Rapp 1994). Some diseases, although devastating, may be too rare to command widespread support for testing; others may be most prevalent among racial or ethnic groups that lack political or economic power. The alternative is to introduce some central policy-making into the development of tests and their introduction into society. The obvious worry here is that the policy will not be sufficiently sensitive to the concerns of citizens, and that it will develop into a system of eugenics. The difficulties raised here are explored somewhat further in section 8.

\subsection{Population-wide testing}

When genetic tests become widely available, and when those tests can be used to predict a future phenotypic condition (either for a fetus or for a person), there will be the possibility of recommending that such tests be taken either by everyone in the population or by people in particular subgroups. Assuming, in light of the discussion of $\S 4.7$, that people will not typically be required to take such tests, it is important to understand when widespread testing will promote some social good.

The consequences of the broad availability of a test depend on a number of important parameters. Technical Note 2, at the end of this section, provides some detail to support conclusions about two possible cases. One, for which colon cancer might be a paradigm, involves predictive testing for an individual. The expected benefits, in economic terms, can be thought of as the economic effect of the continued 
productive life of the person tested. The other, illustrated by testing for Fragile $\mathrm{X}$ syndrome, brings economic benefits through the savings of the costs of care for those afflicted with the syndrome.

The economic perspective on testing programs is not the only one, and, in general, it is important to juxtapose an economic analysis with a more nuanced study of the effects on individual welfare. Some programs that are costly may be worth pursuing because of the suffering that they avoid. Others that make economic sense may have social consequences that are quite damaging.

Some proponents of the HGP have suggested that the project will pay for itself because of the provision of genetic tests in one (or a few) prominent instances: Fragile $\mathrm{X}$ syndrome is a favorite example here. As Technical Note 2 shows, this is far from clear. If Fragile $\mathrm{X}$ testing were carried out across the entire population, then the cost of administering the tests would outweigh the savings in terms of reductions in costs of care for those with the syndrome only if more than half the prospective parents whose fetuses test positive were prepared to terminate the pregnancy. Perhaps this would be so, although the variability of the syndrome and the current lack of reliable statistics connecting genotypes with phenotypes would surely make the decision a harrowing one for any parents who recognized these points. The costs of testing could, of course, be reduced by making testing available in those extended families in which children with Fragile $\mathrm{X}$ syndrome have already been born. Under these circumstances, it is still by no means certain that the testing program would recoup any significant portion of the expenses of the HGP.

Many parents find it hard to consider aborting a fetus that has a condition already present in one of their other children, or in one of their close relatives (OTA 1992 127). To terminate the pregnancy seems to deny what they seek to affirm: the value of those who are born afflicted with the condition. Any program for offering 
pre-natal testing to families in which a genetic condition is recognized must honor two fundamental principles:

(I) The decision must be made by the prospective couple, and it must not be coerced through the transmission of any social attitude that they are blameworthy for burdening society with "defective offspring".

(II) Those who are born must be given equal respect and social support for their distinctive needs.

Unless these principles are honored, the net social effect of making testing available to those at risk of bearing offspring with debilitating conditions will be either to force what should be a free decision or to worsen the predicament of those who already suffer because of their genotypes.

It is entirely right and proper that there should be public discussion of the prospects for people who bear particular genetic conditions, and that prospective parents should take into account the demands that their decision to bring into the world a child with one of these conditions will make on other members of society. But the discussion and the reflection must take place in a context that honors the parents' rights to obey their own fundamental convictions and values, and that recognizes each person as worthy of equal respect. Unless that context can be achieved and maintained, it is likely that efforts to avoid suffering by making pre-natal testing broadly available to those especially at risk will actually produce suffering, through the stigmatization of those who decline to undergo the tests and those who are unlucky enough to be born with the conditions that the tests seek to prevent (Hubbard and Wald 1993).

Disability activists have been especially vocal in opposing widespread practices of pre-natal testing. They fear that a program to lower the incidence of a genetic condition (Down syndrome, Fragile X syndrome) will worsen the plight of those who are born with these conditions, both in terms of the withdrawal of support (will we continue to work hard to alleviate the predicaments of those whose births we try to 
prevent? - see (OTA 1992) 52, (Paul 1991)) and in terms of the loss of respect (can we continue to respect people when we try to prevent the birth of more like them? -see (Hubbard and Wald 1993)). Neither consequence is strictly necessary. The treatment of cases of thalassemia in Cyprus, where a program of genetic screening administered by the Greek Orthodox Church has diminished the incidence of births of children with thalassemia, shows that it is possible to combine pre-natal testing with increased support for those who suffer: because there is now less demand for blood transfusions and chelating treatments, the lot of thalassemia sufferers is now far better than it was (Angastiniotis et.al. 1986). Nonetheless, there are legitimate reasons to wonder whether similar social support would be available in a less cohesive, more ethnically diverse, more anonymous society such as the US. The second implication can also be questioned. It is possible to recognize that the suffering and frustration that would attend the lives of those born with a particular genetic condition would justify efforts not to bring into being people with that condition, while simultaneously seeing that, once such people are born, they are worthy of equal respect and of the greatest support that we can give them to develop their lives in relatively pain-free and fulfilling ways. From the situation of the prospective parents, in which the people do not yet exist -- the lives "have not yet begun in earnest", in Dworkin's apt phrase -- the appropriate moral perspective is one in which efforts are made to make the world better in terms of the quality of the lives that are led in it, and so it is morally permissible to act so as to prevent suffering and stringent limitations. Once lives have begun "in earnest", however, this is no longer the appropriate moral stance, for there are people whose rights and interests must be considered, and these people are worthy of our respect and support. Nevertheless, while it may be possible to develop this delicate balance between two different moral perspectives, it is surely legitimate for those with disabilities to wonder if the balance will be maintained within US society, and to suspect that the loss of equilibrium will be to their social detriment. 
The debate over programs to make widely available pre-natal testing for genetic conditions is more complex than is usually realized. Considerable suffering could be alleviated if the incidence of babies with Tay-Sachs, Lesch-Nyhan, Fragile X Syndrome, and a number of other conditions, was reduced. In principle, as in the Cypriot management of thalassemia, resources saved could be expended to support those born with genetic disabilities. Respect for the disabled could be maintained. Yet, there is reason for those who suffer from genetic disabilities to worry that prenatal screening will cause further suffering for those who are born with disabilities. It would be tragic if we were to forego the genuine benefits that pre-natal testing can bring, but, equally, tragic if we were to purchase those benefits at cost to those who already face severe obstacles. Although the solution, both in terms of practical action and in terms of social attitudes, is precarious, it is surely worth struggling to ensure that both principles (I) and (II) are honored within US society.

\subsection{Summary of main conclusions}

Because the discussion is complex and ranges over many issues, it is worth summarizing some main points.

- The first fruits of the HGP will be genetic tests, and the HGP will greatly expand the range of genetic tests available.

- Genetic testing can be employed in a number of contexts, Diagnostic (initial and disambiguating), and Predictive (risk of disease, pre-natal testing of fetus, testing of prospective parents).

- Proposed genetic tests need to be assessed with respect to each context in which they might be employed. Statistical information is needed for predictive contexts that is not needed in the nonpredictive contexts.

- For the predictive contexts, the most direct way to generate the pertinent information is to sample the general population. It is important to consider 
different types of environments when known environmental differences affect the phenotype.

- In many diagnostic and predictive contexts a rich background of counselling support is necessary. This is especially evident in those instances in which little can be done to treat a disease.

- Equal access to effective counselling needs to be assured. Because current studies show difficulties of communication in counselling situations, attention should be given to developing programs that will enable counsellors to transmit the information people need.

- Mandatory testing is only legitimate when a refusal to take a test would cause irremediable harm to others. Ideally, tests should respond to the most urgent needs of the whole population, but there are serious questions about how these needs should be assessed.

- Programs that make testing widely available should be judged both in terms of their economic consequences and in terms of their social effects. The economic implications of some programs considered (see Technical Note 2) are not clear. There are legitimate social concerns about such programs, and these demand a context in which both individual reproductive choices and people born with disabilities are properly respected. 


\section{TECHNICAL NOTE 1}

\section{The XYY Fallacy}

Because of the way in which disease genes are currently identified, we often have information about the probability that someone with a phenotypic condition, $\mathbf{P}$, has a disease gene, $\mathbf{G}$-- in other words, we know $\operatorname{Pr}(\mathbf{G} / \mathbf{P})$. Frequently, this is close to 1 .

What the patient, or prospective parent, needs to know is $\operatorname{Pr}(\mathbf{P} / \mathbf{G})$ [the probability that someone with the genotype has the phenotype]. As noted in $\$ 4.1$, what is typically needed is a set of values $\operatorname{Pr}\left(\mathbf{P} / \mathbf{G} \& \mathbf{E}_{\mathbf{i}}\right)$ where the $\mathbf{E}_{\mathbf{i}}$ are the environments that are available. The more complicated problem of computing these values are fundamentally similar to the simpler case of finding $\operatorname{Pr}(\mathbf{P} / \mathbf{G})$.

The $X Y Y$ Fallacy consists in inferring from the fact that $\operatorname{Pr}(\mathbf{G} / \mathbf{P})$ is high that $\operatorname{Pr}(\mathbf{P} / \mathbf{G})$ is also high. In general it is possible that $\operatorname{Pr}(\mathbf{G} / \mathbf{P})=1$ and $\operatorname{Pr}(\mathbf{P} / \mathbf{G})$ be low. This is shown empirically by the case of the XYY karyotype. It is easy to see in general. For, by Bayes' Theorem:

$$
\operatorname{Pr}(\mathbf{P} / \mathbf{G})=\frac{\operatorname{Pr}(\mathbf{P}) \operatorname{Pr}(\mathbf{G} / \mathbf{P})}{\operatorname{Pr}(\mathbf{P}) \operatorname{Pr}(\mathbf{G} / \mathbf{P})+\operatorname{Pr}(-\mathbf{P}) \operatorname{Pr}(\mathbf{G} /-\mathbf{P})}
$$

For a rare disease phenotype, in which $\operatorname{Pr}(\mathbf{P})=1 / 5000, \operatorname{Pr}(\mathbf{G} / \mathbf{P})=1, \operatorname{Pr}(\mathbf{G} /-\mathbf{P})=q$ :

$$
\operatorname{Pr}(\mathbf{P} / \mathbf{G})=\frac{2}{2+10,000 q}
$$

When $q=1 / 100$ (only one person in a hundred without the phenotype has the genotype), $\operatorname{Pr}(\mathbf{P} / \mathbf{G})<1 / 50$.

\section{Calculating the Probabilities}

In the standard practice of human genetics, we do not just know $\operatorname{Pr}(\mathbf{G} / \mathbf{P})$, but also $\operatorname{Pr}(\mathbf{P} / \mathbf{G} \& \mathbf{F})$ where $\mathbf{F}$ is the property of belonging to one of the families in which the gene has been identified. When can $\operatorname{Pr}(\mathbf{P} / \mathbf{G} \& \mathbf{F})$ be taken as an estimate of $\operatorname{Pr}(\mathbf{P} / \mathbf{G}) ?$ 
By the theorem on total probability,

$$
\operatorname{Pr}(\mathbf{P} / \mathbf{G})=\operatorname{Pr}(\mathbf{F}) \operatorname{Pr}(\mathbf{P} / \mathbf{G} \& \mathbf{F})+\operatorname{Pr}(-\mathbf{F}) \operatorname{Pr}(\mathbf{P} / \mathbf{G} \&-\mathbf{F})
$$

Because $\operatorname{Pr}(\mathbf{F})$ is very small in comparison to $\operatorname{Pr}(-\mathrm{F}), \operatorname{Pr}(\mathbf{P} / \mathbf{G})$ will be closely approximated by $\operatorname{Pr}(\mathbf{P} / \mathbf{G} \&-\mathbf{F})$. So it is only correct to estimate $\operatorname{Pr}(\mathbf{P} / \mathbf{G})$ by $\operatorname{Pr}(\mathbf{P} / \mathbf{G} \& \mathbf{F})$ if $\operatorname{Pr}(\mathbf{P} / \mathbf{G} \& \mathbf{F})$ is very close to $\operatorname{Pr}(\mathbf{P} / \mathbf{G} \&-\mathbf{F})$. To suppose this, one must believe that there are no features associated with being $\mathbf{F}$ (i.e. being a member of one of the families tested) that make a substantial causal contribution to the presence of the phenotype. However, in cases in which the phenotype is variable (cases of variation in penetrance), one knows that there are unknown factors that make a substantial contribution to the phenotype -either background genetic conditions or environmental factors. Further, it is also known that the families initially identified for tracking the disease gene are those that have special combinations of background factors that make the genotype-phenotype relationship especially easy to follow. Under the circumstances, it is reasonable to think that $\operatorname{Pr}(\mathbf{P} / \mathbf{G})$ may be substantially different from $\operatorname{Pr}(\mathbf{P} / \mathbf{G} \& \mathbf{F})$.

\section{Calculating $\operatorname{Pr}(\mathbf{P} / \mathbf{G})$ directly}

$\operatorname{Pr}(\mathbf{P} / \mathbf{G})$ can readily be calculated by finding the frequency with which people who do not have the phenotype bear the genotype, $\operatorname{Pr}(\mathbf{G} /-\mathbf{P})$. Consider the case of Fragile X syndrome, where the phenotype (mental retardation and the other symptoms) occur with a frequency of $1 / 2000$. Assume that $\operatorname{Pr}(\mathbf{G} / \mathbf{P})=1$. If we now discover that, among 10,000 asymptomatic people tested, none carries the $\mathrm{Fra}(\mathrm{X})$ allele, it is possible to substitute in Bayes' Theorem, obtaining:

$$
\operatorname{Pr}(\mathbf{P} / \mathbf{G})>\frac{1 / 2000}{1 / 2000}+1 / 10,000
$$

so that $\operatorname{Pr}(\mathrm{P} / \mathrm{G})$ can safely be estimated at greater than $5 / 6$. 


\section{TECHNICAL NOTE 2}

Population-wide pre-natal testing

Suppose that there are $\mathbf{N}$ births each year, that the probability of the condition for which the test is given is $\mathbf{p}$, and that the cost of lifetime care for each person born with the condition is $\mathbf{C}$. Then the total cost of care due to a year's worth of births is pNC.

Imagine that tests are offered across the entire population. Let the probability that a couple will choose the test be $\mathbf{q}$, the probability that the condition is present if the test gives a positive result be $\mathbf{r}$, and the probability of choosing to terminate the pregnancy, given that the test discloses a positive result, be s. Suppose that the cost of testing per case is $\mathbf{D}$. The number of births per year with the condition will be $\mathbf{p}(1-$ qrs)N. The total cost due to a year's worth of births will be: qND + p(1-qrs)NC. Widespread testing is worthwhile (economically) if

$$
\begin{aligned}
& \mathbf{q N D}+\mathbf{p}(1-\text { qrs }) \mathbf{N C}<\mathbf{p N C} \\
\text { i.e } & \mathbf{C} / \mathbf{D}>1 / \text { prs. }
\end{aligned}
$$

Henceforth imagine that $\mathbf{r}=1$ (the tests always disclose the condition when it is present). Then the condition for testing to be worthwhile is $\mathbf{C} / \mathbf{D}>1 / \mathrm{ps}$.

For Fragile X Syndrome:

$$
\begin{aligned}
& \mathbf{C}=\$ 1.6 \mathrm{M} \\
& \mathbf{D}=\$ 400 \\
& \mathbf{p}=1 / 2000 .
\end{aligned}
$$

So, if $\mathbf{C} / \mathbf{D}>1 / p s$, then $\mathbf{s}>1 / 2$. This means that termination has to be chosen more frequently than one in every two cases.

When $\mathbf{C} / \mathbf{D}>1 / \mathrm{ps}$, the amount of savings is represented by: $\quad$ qN(psC $-\mathrm{D})$. Suppose that $\mathbf{s}=3 / 4$, and that $\mathbf{N}=5,000,000$. Then the savings, per year of births, would be \$q billion. If the rate of taking the test were sufficiently high -- greater than $3 / 10$ - then the costs of the HGP would indeed be recouped in a decade. This is 
presumably the scenario envisaged by those who believe that pre-natal testing will enable the HGP to pay for itself. Plainly empirical research is needed to determine if the assumptions about the crucial parameters, $s$ and $q$, is realistic. (One might argue that $\mathbf{S}$ is likely to be relatively high, on the grounds that those who take the test are, presumably, contemplating abortion given a positive result. However, there is considerable evidence that couples change their minds about what they will do when faced with the actual test findings.)

\section{Testing a sub-population}

Suppose now that we restrict our attention to a subpopulation of size $\mathbf{M}$, in which the probability of the condition, $\mathbf{p}^{*}$, is significantly greater than $\mathbf{p}$. The total cost of care due to a year's worth of births in the subpopulation is $\mathbf{p}^{*} \mathbf{M C}$.

If the probability that a member of the subpopulation will take the test is $\mathbf{q}^{*}$, and the probability of terminating pregnancies that test positive is $s^{*}$, then the number of births with the condition within the subpopulation is $\mathbf{p}^{*}\left(1-\mathbf{q}^{*} \mathbf{s}^{*}\right) \mathbf{M}$. The total cost due to a year's worth of births within the subpopulation is

$$
\mathbf{q}^{*} \mathbf{M D}+\mathbf{p}^{*}\left(1-\mathbf{q}^{*} \mathbf{s}^{*}\right) \mathbf{M C} \text {. }
$$

Testing is economically worthwhile provided that

$$
\text { C/D }>1 / \mathbf{p}^{*} \mathbf{s}^{*}
$$

and the savings when this obtains are

$$
\mathbf{q}^{*} \mathbf{M}\left(\mathbf{p}^{*} \mathbf{s}^{*} \mathbf{C}-\mathbf{D}\right) \text {. }
$$

Perhaps it would be possible to choose a population for testing of size approximately 100,000 , within which the probability of Fragile X Syndrome is $1 / 50$. (Our previous figures estimate 2,500 Fragile $\mathrm{X}$ births per year, and the assumption is that 2,000 of these (80\%) might be concentrated in 100,000 instances. If this were so, then for testing to be cost-effective it would have to be that

$$
s^{*}>1 / 80
$$


It seems overwhelmingly probable that this condition would be met!

Supposing, that $s^{*}=1 / 4$, the extent of the savings would now be $\$ 760 q^{*} \mathrm{M}$. If $q^{*}>4 / 9$, the costs of the HGP would be recovered in a decade.

\section{Testing for future diseases}

Assume that $90 \%$ of cases of a particular disease can be traced to the presence of one of a set of alleles, for which people can be tested. Let the costs associated with not treating the disease early (stemming from the expense of later interventions and of losses due to incapacity or fatality) be $\mathrm{C}$. Let the frequency of the disease in the population of size $\mathbf{N}$ be $\mathbf{p}$. Without testing, the costs for the present population are pNC.

Let the cost of the test be $\mathbf{D}$. If people are tested and found positive, they may be monitored at a cost $\mathbf{E}$. Monitoring gives a probability $\mathbf{q}$ of early detection. Given early detection, an intervention may be undertaken at $\operatorname{cost} \mathbf{F}$, with probability $\mathbf{r}$ of complete success. Assume that complete success brings no further costs, but that failures of detection or in intervention bring the further cost $\mathbf{C}$. Suppose people choose to take the test with probability $\mathbf{s}$, and that all those who take the test and are found positive are monitored.

Given testing, the expected number of cases of the disease is computed as follows. $0.9 \mathrm{pN}$ instances arise from the presence of one of the alleles. $0.9 \mathrm{psN}$ will be discovered through testing (assuming that the test always discloses one of the genetic conditions when it is present). 0.9 pqrsN will be detected.

The total costs will be:

$$
\begin{aligned}
& 0.1 \mathbf{p N C}+0.9(1-\mathrm{s}) \mathrm{pNC}+0.9 \mathrm{~s}(1-\mathbf{p}) \mathrm{ND}+0.9 \mathrm{sp}(1-\mathbf{q}) \mathbf{N}(\mathbf{C}+\mathrm{D}+\mathbf{E})+0.9 \mathrm{spq}(1- \\
& \mathbf{r}) \mathbf{N}(\mathrm{C}+\mathrm{D}+\mathrm{E}+\mathbf{F})+0.9 \operatorname{spqrN}(\mathbf{D}+\mathrm{E}+\mathbf{F})
\end{aligned}
$$

If these are to be less than the cost without testing, pNC, then it must be the case that 


$$
\text { pqrC }>\mathbf{D}+\dot{\mathrm{pE}}+\mathbf{p q F}
$$

When $\mathbf{C}$ is large in comparison to $\mathbf{D}, \mathbf{E}$, and $\mathbf{F}$, and $\mathbf{q}$ and $\mathbf{r}$ are reasonably close to one (the monitoring procedures and the interventions are quite successful), then it is easy to see that testing can be economically worthwhile.

In the case of colon cancer, if we suppose that $p$ is $0.01, q$ and $r$ are each 0.8 , that $\mathrm{C}$ is $\$ 400,000, \mathrm{D}$ is $\$ 400, \mathrm{E}$ is $\$ 15,000$, and $\mathrm{F}$ is $\$ 10,000$, the inequality becomes

$$
2560>400+150+80
$$

which is clearly satisfied. Thus if these estimates are even approximately correct, there are obvious economic benefits from encouraging widespread testing.

As noted in the text, economic analyses should always be juxtaposed with social analyses. Here, if anything, the social analyses seem to provide a more favorable conclusion in that the dollar figure placed on costs of leaving the disease untreated -- a consequence that will frequently lead to premature death -- seems a crass and ludicrous underestimate. Moreover, in this example, unlike the more vexed cases of prenatal testing, damaging social side effects are far less evident. (For a similar approach to a concrete case, with considerable detail, see (Elias et.al. 1992)). 


\section{The Threat of Genetic Discrimination}

\subsection{Two sources of the value of privacy}

Many commentators on the HGP have taken it for granted that the information generated from the project might play a valuable role in leading to the development of genetic tests, but they have worried about the consequences of generating genetic information about individuals. The tendency has been to think that the central issue in the HGP, and more generally in any applications of contemporary molecular genetics, is the privacy of genetic information. For those who share this assumption, the big questions are: "Who controls genetic information? Who should have access to genetic information?" It is an elementary point that genetic information is only of use to the people tested if others share the information: counsellors, doctors, sometimes other family members have to know the genetic facts about the subject. These disclosures are taken to be unproblematic because those who have the knowledge are taken to have the subject's well-being at heart.

The worry is surely that genetic information will spread further, to people whose interest in the subject is not so benign. Now it is relevant to note, from the outset, that genetic information cannot be kept completely private. Anyone who knows enough about a subject's family can infer, often only in a rough and ready fashion, some probable characteristics of the subject's genotype. Part of the standard practice of reviewing applications in a variety of contexts has been to ask for details of family history. Why, then, should people be concerned about the transmission of accurate information?

Reflection on these points brings home the fact that there are two quite general types of reasons for valuing privacy, when what is kept private is information about ourselves. Privacy may have instrumental value, in that knowledge of information could be used as a means to cause us some further, independent, harm. It may also have intrinsic value, in that knowledge of information would, in itself, independently 
of what uses are made of the information, be harmful to us. The difference is between not wanting others to know that we bear a particular combination of alleles, because that would provide them with the opportunity for doing things that are to our detriment, and simply not wanting them to know, whatever else they then go on to do. Now the current tolerance for the use of family history questionnaires can best be understood as embodying the thought that not much information that is both precise and can be relied on with any confidence can be gleaned from them, and that, therefore, those who use the questionnaires are unlikely to do a serious amount of harm. This seems to point to the fact that what is of greatest concern, when we think about the privacy of genetic information, is the instrumental value of privacy.

There are some things about ourselves that we do not want others (with the exception of our intimates) to know, but most of the facts about our genotype do not fall into this category. If molecular genetics were able to disclose the characteristics of our behavioral propensities (revealing, say, our sexual orientation), then this might seem information that we want to keep to ourselves irrespective of what others do with it. But, as section 3 has already suggested, and as section 8 will re-emphasize, these kinds of findings are not at all likely in the foreseeable future. Our immediate concerns should be with information of a far more mundane type -- whether a person is at increased risk for heart disease or cancer, whether someone is susceptible to the effects of a workplace environment. We do not regard this kind of information as having some intrinsic value of privacy. The trouble lies in what can be done with it, given current US social institutions.

Although many scientists and commentators on the HGP have suggested that we need a "genetic privacy act", the foregoing considerations indicate that no such act may be needed. To be sure, if we could formulate a policy for limiting access to genetic information, that would allow those who are committed to helping a person to have genetic information about that person, while denying the information to others whose 
interests potentially conflict with those of the subject, then a very large class of problems surrounding the production of genetic information would be resolved. Yet, so long as we do not restrict the use of any information that is acquired, outsiders may have an interest in discovering things which they may use to a subject's disadvantage. Initially, controlling the flow of information seems the more direct way of avoiding abuses of genetic findings, but we could protect the instrumental value of privacy of information by inhibiting the uses that can be made of it. Indeed, this may be the central part of the most effective strategy: controlling information is extremely difficult if those whom we do not want to have access have excellent reasons for trying to find out. There are serious problems in safeguarding confidential records, especially when these are kept in computer files, and, furthermore, there is no easy way to monitor the flow of information until that information is used to cause someone trouble. By restricting the use that can be made of genetic information, we remove much of the motivation to acquire such information, thus making problems of control more tractable. Equally, we would have procedures for responding to breakdowns in the control of information as soon as they become visible. If this is correct, a "genetic privacy act" is not primarily what is needed to solve the problems that have, quite rightly, been discemed in the compilation of genetic information about individuals -although it may be useful as a supplement to measures designed to restrict what can be done with genetic information (for valuable discussions of privacy, see (Thomson 1975, Scanlon 1975, Reiman 1976).

There are some difficulties that arise in medical and legal contexts, which cannot be addressed by the approach recommended below. Doctors sometimes face dilemmas about divulging information to the relatives of their patients, and resolving these dilemmas does demand attention to issues about privacy. But these are general questions that are by no means specific to the HGP, that are rightly discussed within 
the context of the professional ethics of medicine, and that do not call for social policy decisions.

The central problem for discussion below is: How can we prevent genetic information about a person from being used to harm that person unjustly? This question can be adequately answered if it is possible to survey the various possible ways in which information can be abused, so that the concerns about instrumental value of privacy can be addressed. The central topic is genetic discrimination, for knowledge of a person's genotype can only be employed unjustly to harm the person if there are recognized institutions, or ways of treating people, that vary with the assignment of people to genetically characterized classes. The task is to consider the variety of contexts in which people might be classified on the basis of knowledge about their genotypes, to recognize those instances in which the classification causes unjust harm to the members of some of these classes, and to formulate restrictions on actions so as to prevent these harms. For it is abundantly clear that there will be no value in genetic testing so long as people fear that test results will be used to discriminate against them.

\subsection{Contexts of Genetic Discrimination}

The potential for harmful genetic discrimination exists in a number of current contexts: in evaluating applicants for insurance - particularly health, life, and disability insurance; in assessing candidates for job positions and training programs; and in assigning jobs to those who are already employed. There is also a general, more nebulous, context of what might be termed "social evaluation", and some concerns about uses of genetic knowledge in this context surely underlie the fear that people with particular genotypes will be stigmatized (Hubbard and Wald 1993). Genetic discrimination might proceed independently of racial, ethnic, or sexual discrimination, 
or it might reinforce existing forms of discrimination. These issues will be considered separately.

Genetic discrimination arises only when those making evaluations and assignments perceive it to be useful for them to distinguish classes that are genetically characterized, to identify which people belong to which classes, and to treat the members of different classes differently. But not all ways of making these distinctions are unjust. Nor, when genetic discrimination does occur, when the classification and resultant differential treatment is unjust, are the procedures that are followed more unjust that procedures that have nothing to do with genetic information. Although the following discussion will confine its attention to matters of genetic discrimination, it is worth noting that the principles used to identify cases of discrimination might have broader implications.

The threat of discrimination arises most clearly in employment and insurance contexts, because, in some circumstances both employers and insurers can benefit from knowledge of the genetic characteristics of applicants. Assuming that genetic information can predict risks of future disease, insurance companies can use it to adjust premiums or even to refuse insurance to those whose risks they deem too high. Similarly, employers can gain advantages by knowing which of their potential employees are at high risk of becoming incapacitated, and how the risks of sickness and disability vary with workplace environments.

There are three main kinds of action that an evaluator can take, based upon knowledge about the genetic characteristics of a subject. Some actions would be beneficial to the subject, irrespective of the class to which the person is assigned. Other actions are to the disadvantage of members of some classes, but are not unjust. Finally, there are actions that unjustly harm the members of some classes. Examples of each kind are easily envisaged -- although the simplest cases are unrealistic and ignore the market constraints that affect the behavior of employers. First, an employer 
might use genetic information to benefit the members of all the classes disinguished, by assigning people to equally lucrative jobs with workplace environments which, given their genotypes, were most conducive to their health. Second, an employer might deny some people the opportunity to train for a well-paying and prestigious post, on the grounds that those people bear an allele that causes them always to collapse in the environment associated with the position, in such a way that they would become a danger to others. Finally, an employer might fire all those who carry a particular genotype, not because that genotype affects their performance in the workplace in any way, but because the genotype is associated with a quite extraneous characteristic that the employer dislikes. These three pure cases illustrate the range of possibilities: benefit, harm without injustice, and unjust harm. The task of assigning real-world examples to these categories is far more difficult. (There are, of course, economic reasons for thinking that employers will be penalized for using the more capricious forms of discrimination.)

\subsection{Misinformation and Injustice}

One question, concerning the use of faulty information, cuts across the contexts just distinguished. If an evaluator uses a system of classification to differentiate subjects, the classification of an individual may be accurate or inaccurate. In some circumstances, assignment of a person to a class may cause that person to be harmed. Under what circumstances, when the assignment is incorrect, has an injustice been done to that person? Suppose, for example, that you are denied a job because you are assessed, on the basis of a genetic test, as being at high risk for developing a cancer that will terminate your work prematurely, and the conclusion about your risk is incorrect. Waiving the question whether it would have been just to deny you the job if you had been correctly assessed -- a question considered in $\$ 5.6$-- does the mistaken assignment of risk constitute an injustice? 
Past uses of genetic findings have caused harm in insurance and employment settings. To cite one famous example, the Department of Defense refused to admit individuals with sickle-cell trait (heterozygotes for the normal allele and the sickling allele at the crucial globin locus) to the Air Force Academy, thereby blocking them from the most direct route to positions as commissioned officers in the Air Force (Duster 1990, 26-7). The official justification for this policy was that people with sickle-cell trait are at high risk of collapse at high altitudes; however, there is no biological justification for this conclusion. In the US population, the sickling allele -and thus both sickle-cell trait and sickle-cell anemia -- is most prevalent among American blacks. This appears to be an example in which genetic differentiation interacts with older forms of discrimination (see $\$ 5.8$ below).

Insurers and employers, like other people who make use of technical information, are bound to make mistakes. Sometimes even the best and most authoritative scientific findings are incorrect, and, though application of those findings may do harm, those who rely on them cannot be blamed. However, the example of admission to the Air Force Academy is not like this: the genetics of sickle-cell anemia had been extensively studied for thirty years before the Department of Defense was forced, by lawsuit, to revoke its policy. Not all ignorance is culpable, but not all is blameless. When applications of scientific information are made in ways that cause some people harm, it is the responsibility of those making the-applications to ensure that they thoroughly understand the relevant pieces of science and that they are acquainted with the research that bears on the situation.

Given the discussion of $\$ 4.4$, it is clear that there are dangers that the results of genetic tests will be unthinkingly applied. Without the compilation of statistics for the general population, assignments of risk are vulnerable to error. If the calculations of risk fail to take into account known relevant environmental differences, then people with quite different levels of risk will be classified together. Since these general 
sources of error are well known, it is right that those who intend to make use of genetic findings should be aware of them, and that they should take care that efforts have been made to avoid them.

These considerations suggest an Informational Precondition on the use of genetic tests to classify people and to treat the classes differently:

Those who apply the test must understand

(i) the range of variation at the locus and the range of variation in the phenotype for each combination of alleles at the locus,

(ii) the probabilities of each phenotype, given each genotype, differentiated insofar as is possible according to known environmental variables.

If the informational precondition is violated, then, whatever the outcomes, responsible procedures have been violated, and those harmed can legitimately demand reparations.

Henceforth, the discussion will take it for granted that the informational precondition is satisfied, and consider questions of justice under the assumption that genetic information has been thoroughly digested (and, indeed, that that information is correct).

\subsection{Health Insurance}

If an insurer knows that certain genotypes are associated with certain risks, then also knowing the genotypes of individuals in the applicant pool will make it possible to set premiums in ways that make it probable that claims will be covered. If the insurer does not have the knowledge, but the applicants do, then it is possible for the applicants to generate a distribution of premiums and claims that would be ruinous for the insurer, not because they want to cause that ruin, but simply because it may be advantageous for those at higher risks to insure for larger amounts. Since the insurer has no basis for not setting equal premiums (or, at least, for not making the distinctions that the 
applicants can make), claims are likely to outrun premiums. (This is one version of the phenomenon of "adverse selection" in which those at higher risks try to insure for greater amounts, provided that they are not charged disproportionately higher premiums for doing so.)

Recognition of these possibilities generates two lines of argument for letting insurers use genetic information in setting premiums.

(I) Insurance underwriting is the business of setting premiums on the basis of risk. Standard practices use estimates of risk that may be misleading. The proposal to use genetic information should lead to a more accurate assessment of risks. If risks are assessed more accurately, then they are assessed more fairly. In consequence, genetic testing will lead to a fairer set of premiums than those currently fixed.

(II) For any market in services, potential consumers should not have resources that would make it possible and advantageous for them to act in ways that would, with high probability, cause the ruin of the suppliers. (Markets populated by rational agents should not be self-undermining.) Not to allow genetic information to insurers and to allow it to applicants for insurance would generate such resources for the consumers. Because people have to be permitted access to genetic information about themselves, that information must also be permitted to insurers.

These arguments have been stated quite generally, so that they apply to various types of insurance. Norman Daniels (forthcoming) has offered a cogent critique of (I) in the special context of health insurance. Daniels points out that the notion of fairness is ambiguous between the actuarial (or statistical sense), in which it is a truism that fair premiums must accord with the actual risks, and the sense pertinent to issues of justice, in which supposing that fair premiums accord with the actual risks begs important questions. It would be fallacious to slide from the claim that certain premiums are 
actuarially fair to the conclusion that those premiums are just, and (I) only appears plausible because the same term is employed in two different senses.

Nevertheless, by considering the argument in its general form, it is possible to see that there are sometimes ways of making the connection between actuarial fairness and justice. When what is at issue is the insurance of household valuables, the habits of a family seem quite pertinent to determining the premium that should be paid. If one family is gregarious, hospitable, somewhat careless, and rather absent-minded, while a second is neat, punctilious about locking doors, and fussy about the moral probity of people in its social circle, it is not simply a matter of actuarial fairness but of fairness in the sense of justice to insist that fair premiums should charge the first family more.

Does it make a difference when the topic is health (not family valuables) and the information concerns genetic characteristics (not habits of behavior)? There are two main sources of disanalogy. One is the special role that health, and the maintenance of health, plays in people's lives, which gives rise to the conception that requiring high premiums of people, even denying them access to health insurance (thus greatly affecting their opportunities for treatment, prevention of disease and maintenance of health), is to curtail or to eliminate a capacity fundamental to planning and living one's life. The second is the difference between those facets of our behavior that we can change (as, we imagine, the first family could change its warm-hearted but unfastidious ways) and those conditions we are born with. While it may be fair to demand a higher price from people who could join the class of those offered a cheaper bargain, if they chose to do so, it does not seem just to penalize people for characteristics that they cannot change.

These differences make a powerful case for limiting the ability of medical insurers to refuse coverage or to impose heavier premiums on those who are known to have particular genetic conditions. The Constitution was surely framed in full 
recognition of the variety of goals that citizens may set for themselves, the diversity of ways in which they may "pursue happiness", and it tries to protect individual freedom to formulate and pursue a good and valuable life. Within this framework, a major task of political theory is to try to resolve those dilemmas which arise when one person's plans and projects clash with those of another. Faced with these dilemmas, we rightly look to the relative urgency of the demands that would be met by the various courses of action which the agents involved intend. In those instances in which a course of action envisaged by one deprives the other not only of the opportunity to carry out some particular project, but impedes the pursuit of any coherent life plan, and in which the situation is not symmetrical, there are grounds for resolving the conflict in favor of the person who is threatened with the loss of the opportunity to pursue any kind of happiness. Other things being equal, there is a duty to protect those who are threatened with the loss of resources that would be needed to pursue any life goals. Arguably, the maintenance of health belongs among those resources, so that the conflict between insurers and applicants for insurance should be resolved in favor of the applicants.

Other things may not be equal. Sometimes conflicts come about because one of the parties has now come to occupy an unenviable position because of past actions that were freely taken, in clear understanding of the consequences. When this occurs, there are grounds for changing the evaluation. It would be wrong to start from the present situation, assessing the relative centrality of the resources to the two contending parties. Instead, it is necessary to consider their lives as wholes, recognizing that choice of a plan which, predictably, led to danger of the removal of conditions for its own continuation, forfeited the right to later protection. To descend from the abstract to the concrete case of concern, those who do things that they know will later put them at greater risk of susceptibility to severe health problems cannot complain if insurers hold them to account for the risks they have knowingly and voluntarily incurred. 
There are bound to be borderline cases in this arena, for the conditions of free choice and foreknowledge are not easy to circumscribe. But there are also clear examples: people who, in full knowledge of the medical consequences, overindulge all the wrong habits (although our judgment of these cases might itself be affected if we were to learn that there is a strong genetic component to people's ability to resist tempting bad habits; but see section 8).

However, the discussion of genetic testing in insurance is concerned with the opposite end of the spectrum. If, no matter what an agent had done, a situation of conflict would have come about in which that agent would be threatened with the loss of resources required for pursuing any life plan, then there is a duty to protect that agent. There are clear concrete cases: suppose that someone bears an allele which inevitably leads to high risk, no matter what he does (as, for example, when the person carries a mutant allele that begins the course of a cancer). The need for protection is also clear in other situations, as when there was no information available to the agent that would have led to choice of an environment with reduced risk.

Insurers should thus not be permitted to use genetic information to deprive people of health coverage. Does it follow that they may not use genetic tests to adjust premiums to the degree of risk? Complete adjustment of premium to risk would surely lead, in a significant number of cases, to the imposition of costs that would, in effect, prevent the applicant from obtaining health coverage, so that this would fall afoul of the commitment to ensure the possibility of coverage for all. However, it might be thought just to use genetic testing to adjust premiums in the direction of conforming to risks so long as this was consonant with the subject's ability to pay.

The issues here are complicated, but one guiding principle is surely that people should not be penalized, other things being equal, for their bad luck in the "genetic lottery" (Rawls 1971). A simple proposal is to view medical insurance as a scheme in which each of us participates to ensure ourselves a vital resource, and to adjust the 
costs to variable abilities to pay. Perhaps this could be regarded as a basis, from which further differences in premiums would be introduced in accordance with risks that people voluntarily take on. However, in both the simple or fine-tuned forms, genetic testing would be irrelevant to the fixing of premiums.

There is an intuitive way to motivate the family of proposals that prohibit the use of genetic information in insurance underwriting. A venerable tradition in political theory appeals to the idea of a social contract, and proposes that just institutions are those that would be chosen by rational, free agents in a position in which they do not know their future positions within society (the idea goes back to Rousseau and beyond; for classic contemporary exposition, see Rawls 1971). This approach to justice does not typically concern itself with institutions as specific as the distribution of health care and the levying of costs (but see Daniels 1985). Nevertheless, our present situation with respect to genetic characteristics of ourselves bears an interesting analogy to the predicament of the hypothetical contractors. Within the next decades it will become possible to acquire detailed information about the genotypes of individuals, and health insurance coverage and costs can then be distributed according to a number of different schemes. We know, in advance, that some significant percentage of the population bears alleles that place them at high risks for disease and disability, within the environments which they are going to be able to inhabit, risks that are high enough to render them uninsurable or to demand additional premiums if genetic considerations are to be considered relevant. We can opt for a scheme in which no attention whatsoever is paid to genetic differences: all are assured health coverage, and costs are divided on non-genetic grounds (most obviously according to ability to pay). Alternatively, we can choose a scheme in which the premiums for the genetically fortunate are reduced and the extra costs borne by the genetically unlucky, even for a scheme in which the differences are so great that some people lack the opportunity to purchase health insurance. At present we are genuinely behind a "veil of ignorance" (Rawls 1971, 
Daniels 1985 47). We do not yet know whether we are among the genetically fortunate or whether we have been unlucky. Even though we stand a good chance (approximately 90\%) of being among the fortunate, and even though, on the second type of scheme, the fortunate are offered health care more cheaply, the gain in dollars is surely insignificant compared with the severe restrictions that would be placed upon our life plans if a scheme of that type were adopted. The foolhardiness of trying to save money in this situation ought to bring home to us the injustice of attending to genetic differences in the distribution of resources so fundamental as health care.

However, it is important to come to terms with the argument (II) formulated above. If people were guaranteed the opportunity to buy as much insurance as they desired, irrespective of genotype, paying premiums at the same rates (or at rates based on ability to pay), then there would indeed be a danger that those who discovered themselves to have risky genetic conditions would apply for greater coverage, while those whose genetic test brought good news might settle for less. In this way, the fear that the market might collapse would be sustained.

To overcome this difficulty it is important to distinguish between that basic level of health maintenance that all people need to pursue their projects, whatever those projects may be, and possible supplemental coverage. The argument for making genetic considerations irrelevant must be seen as seeking to spread the costs of basic health care, so that these costs are divided according to principles that do not attend to genetic differences (e.g. according to ability to pay). Once health care policy has set the level at which care will be provided for all - and how to determine this level will not be considered here - citizens may have the opportunity to buy further coverage. In this context, the arguments rehearsed above no longer apply, and it is reasonable to propose that genetic information may be used in fixing premiums. (However, it is worth asking what proportion of the population can be expected to acquire, and make use of, the genetic information that would be relevant to those ruinous choices that 
insurers fear.) By adopting a two-tiered approach of this kind, where the costs at the first tier are guaranteed to be covered, the feared ruin of the market is avoided. Genetic information can be used neither to permit the insurer to ask more of the unfortunate, in the domain of basic care, nor to allow individual applicants to adjust their expenses to their knowledge of their risks (NIH/DOE 1993 2).

\subsection{Life and Disability Insurance}

Uses of genetic tests in connection with life and disability insurance involve many of the same issues. Both disability and premature death are threats to the completion of life plans, both affect the pursuit of a very broad range of individual versions of the "pursuit of happiness". Those who insure against disability seek to prevent a future in which, for all their prudent savings, their resources are insufficient to carry to completion the plans they have carefully made and around which they have shaped their lives. In a less obvious way, those who buy life insurance do the same, for an important part of someone's plan for her life often consists in promoting the well-being of those she loves. Even if we die prematurely, it is important to the success of our lives that those who survive us may flourish. Moreover, our inability to protect against contingencies that may threaten them after our death is, in most cases, important to our happiness while we live. Although not as central as the need for health maintenance, the need to protect ourselves and our families against calamity is of great importance to people with a diversity of projects.

Concrete examples are easy to find. Imagine the parent of two young children, married to a person who, for whatever reason, does not have the opportunity to work at a high-paying job. The couple has struggled to provide opportunities for the children, buying a house in a district in which they have confidence in the schools, and the family is now established in this social and educational setting. If the primary wageearner were to become disabled or to die prematurely, then, without an insurance 
payment to the survivors, the family would have to make dramatic changes in their way of life. All that they have striven for would be lost, and, in addition to the loss directly associated with the death, the family would be uprooted and exposed to all the pressures from which they have, with much forethought and hard work, so far been protected. Unfortunately, the wage-earner is genetically at high risk for a disease that will bring about a premature death, preceded by a period of disability, and, with the introduction of genetic testing into insurance, it is no longer possible for the family to purchase life or disability insurance at rates which they can afford. Because genetic testing is disallowed in fixing premiums for health insurance, medical coverage is assured. But there is no way for the family to stave off the foreseen calamity.

Should there be a system of guaranteed life and disability insurance, to parallel the two-tier scheme envisaged for health care? In this case, the argument is less compelling. The purpose of life and disability insurance is, almost always, to enable people to secure for their loved ones a range of opportunities which at least some members of society -- often a significant number -- do not enjoy. Considerations of justice thus pull in two different directions. On the one hand it is hard not to sympathize with those who have struggled to build opportunities and security for their children, who, because of genetic conditions that are surely matters of accident not desert, cannot do anything to protect what they have worked so hard to achieve. On the other hand, what they aim to preserve is a level of well-being greater than that enjoyed by members of other groups, many of whom are also not responsible for their lack of opportunities. Confining our attention just to the population of those who seek life and disability insurance, it appears wrong to distinguish people on the basis of genetic conditions that are matters of good or ill fortune, permitting some to guard against the destruction of all they have worked for and depriving others of that option. Yet if we switch our focus to the whole of US society, it is clear that there are many people, equally concerned about the futures of those they love, who lack any chance to 
fashion the kinds of opportunities that are widely desired: their children are stuck in depressing, dangerous, and unhealthy environments, without any access to stimulating and nurturing schools. Although the thought that some will be plunged by genetic disclosures into conditions of discomfort and cramped opportunities is poignant, we should remember the many who are born into desolate circumstances from which there is little realistic hope of escape.

The principle underlying the treatment of health insurance was this: When something is a necessary condition for any life plan, then people should be protected against courses of action that would deprive them of that resource. Even if we were to grant that the ability to secure one's future and the well-being of one's descendants is a necessary condition for any life plan, there would still be a difficulty in applying this principle to argue for a scheme that would allow people to buy (at least a minimal amount of) life and disability insurance, independent of their genetic conditions. For a scheme of this type would not provide the pertinent benefit, a secured future, to all members of the population. The envisaged scheme would be a step in the direction of greater justice - middle-class people would not be impeded by genetic conditions, for which they are not responsible, from obtaining something that is very important for them. It is very hard not to want to change a system in which people, who have "played by the rules" all their lives, are victimized by bad luck in the genetic lottery. However, although there is a compelling case for not making genetic distinctions with respect to life and disability insurance, it is important to recognize that in this case, unlike the example of health insurance, the scheme does not make the same benefit available to all (see Daniels 1985226 for a formulation of the general dilemma faced here: the difficulty of correcting local problems against a background of more general inequity). 


\subsection{Discrimination in the Workplace}

The issues surrounding the use of genetic tests in the workplace are complex. Employers might use tests to assess applicants for positions, or to determine the risks for people whom they already employ. They might want to know about someone's general health prospects, in order to avoid hiring somebody who will require a large contribution towards health or disability premiums. Alternatively, they might seek to evaluate risks of disability or disease in a specific workplace environment. We can begin by considering four types of cases, generated by a pair of distinctions: applicants vs. employees, workplace specific risks vs. general risks.

Given solutions to the problems of health and disability insurance discussed above, some reasons for using genetic tests in the workplace will be removed. Employers who must face the prospect of paying insurance premiums for their workers may incur heavy extra expenses if those premiums are adjusted to risks and if they hire people who are at high risk. If medical insurance and disability insurance are available to all without attention to genetic differences, then there will be no incentive to use genetic tests to guard against heavy insurance expenses. Conversely, without solutions to the insurance problems, we may expect employers to wish to compound the troubles of the genetically unfortunate by refusing to hire them. Carrying genes that place one at high risk for disease is already a burden; that burden is increased by demanding higher premiums for those whose genetic conditions put them at risk; and it is increased still further if employers are allowed to use this information to deny work to the genetically unfortunate. It has been argued that justice requires ignoring genetic discrimination in matters of health insurance, and that partial justice requires ignoring those differences with respect to disability insurance. If the demands of justice are met at these levels, employers cannot justify using genetic tests on the grounds that, without those tests, they would become liable for ruinous expenses. This leads to a first conclusion: 


\section{Acknowledging the irrelevance of genetic differences in fixing} insurance premiums enables us to avoid arbitrating many issues of the use of genetic tests in the workplace.

Before proceeding further, it is reasonable to ask whether employers will want to use genetic tests, once they are freed from the prospect of considerable insurancerelated burdens. Genetic tests currently cost figures of the order of $\$ 100$, and it may seem initially that employers would not want to incur the expense of administering tests to large numbers of employees. Two points need to be noted: first, in some markets, employers may ask potential employees to bear the costs of testing, and to provide details about aspects of their genotypes (this point might also apply in the context of insurance, where insurance companies could offer "regular" policies at high premiums and discounts to those willing to volunteer the right type of certified information -such approaches would, of course, be debarred by the proposals of $\$ \S 5.4,5.5$; second, if employers are threatened with costly lawsuits from employees who are affected by substances released in the workplace, their economic interests will be served by incurring the small costs of genetic testing for susceptibilities to those substances (see Draper 1991).

Much discussion of genetic discrimination centers on this last scenario. Those most concerned about the uses of genetic tests by employers frequently contend that such tests shift the proper burden away from those who create industrial settings which put people at risk for ill-health and disability, and on to the individual workers: the strategy is sometimes perceived as one of "blaming the victims" (Nelkin and Tancredi 1989, Hubbard and Wald 1993, Draper 1991). As we shall see below, although there is a very important point here, there are crucial distinctions that must not be overlooked. Sometimes, the workplace can be modified at costs that employers can easily bear, but sometimes either no way of modifying the workplace is known or the costs of those means that are known are so great that they would drive the employer out 
of business (thus, presumably, worsening the predicament of all the workers). Moreover, on some occasions -- although not surely on all, by any means -- workers have options in employment, and those who are genetically susceptible to disease in workplace environments can best serve their own interests by pursuing some of these alternatives.

With these distinctions in mind, it is possible to distinguish the various types of cases that may arise. The general problem is to decide when (if ever) it is permissible for employers to use genetic tests to deny some people access to positions that those people want, on the grounds that those denied carry particular combinations of alleles. The first condition that we should surely set on the use of genetic tests is the Informational Precondition of $\$ 5.3$, restated here as Conclusion 2:

2. Those who apply the test must understand

(i) the range of variation at the locus and the range of variation in the phenotype for each combination of alleles at the locus, (ii) the probabilities of each phenotype, given each genotype, differentiated insofar as is possible according to known environmental variables.

Another relatively straightforward conclusion about uses of genetic tests can be generated by thinking about the differences between testing applicants and testing employees. Intuitively, it might be just to apply a test to someone with whom the employer has no prior contract under circumstances under which it would not be just to apply the test to a person who had already been working for the employer. This can be summarized as follows:

3. Employers typically have obligations to employees that they do not have towards applicants, obligations that are greater, other things being equal, when the period of employment has been longer. These obligations make a difference to the uses that employers can make of genetic tests: 
more stringent requirements must be met in using a genetic test to deny an employee a coveted position than in using a genetic test to refuse a position to an applicant.

Another obvious conclusion comes from considering the possibility that people with particular genetic conditions might endanger the well-being of others, if they were assigned to the positions they seek.

4. It is justifiable to use genetic tests to debar people from positions when those tests identify genetic conditions which would cause their bearers, in the environments associated with the position, to be at appreciably higher risk for acting in ways that would endanger the health of others.

The conclusions achieved so far take us some way towards settling the issues raised by the possibility of genetic testing in the workplace, but they do not touch the genuinely hard cases. To tackle those cases, we need to understand the sources of the conflict between applicant or employee and employer. The first kind of case to be considered will involve tests for a genetic condition that affects health and longevity independently of the workplace environment: the employer plans to use the test to detect general risks. Now there are, in principle, three reasons for which an employer might want to administer a genetic test: (1) to preserve the health of the worker, (2) to avoid losses in worker productivity, (3) to avoid modifying the workplace environment. In the case at hand, because the risk is a general one, (3) is irrelevant. Similarly, because reassignment or workplace modification is irrelevant to the chance that the worker will suffer the threatened consequences, (1) is not pertinent in this case. Hence, if the employer has a case for employing the genetic test and not hiring an applicant or dismissing an existing employee, that case must rest on the right to avoid losses in worker productivity. 
There are some situations in which such a case can be made, and in which an individual's risk for disease is relevant to that person's qualifications for a position. For the moment, the discussion will be restricted to the hiring of applicants for positions. Employers are surely within their rights in considering the likely productivity of potential applicants, and in trying to guard against hiring those whose productivity will be lower. Diminished productivity can come about in one of two ways: through reductions of the time period through which an employee will be able to work, or through lowering of the rate at which the employee is able to work. General risks of ill-health and disability are most obviously relevant to reduced lifespans, and discussion is best focused by imagining that the genetic test is intended to reveal potential losses of productivity through premature inability to work. For example, a genetic test might show that a potential employee is at high risk for a neurological or muscular disorder that will inevitably curtail the person's career, if it should occur.

Employment decisions can be justified by appealing to the principle that the people hired are better qualified for the positions than those who are not -- subject of course to the considerations of affirmative action that qualify the principle of hiring the best qualified. Supposing that affirmative action considerations do not arise in the case at hand (a supposition that will be suspended in $\$ 5.8$ ), it is necessary to ask when judgments about risk of a truncated career are relevant to deciding if an applicant is best qualified for the job.

The clearest instances in which this is so are those in which the employer must provide support for a long period of initial training during which the employee is relatively unproductive, so that training a replacement employee would be costly. Here knowledge about the expected length of the post-training period seems directly relevant to assessing a candidate's qualifications -- although it is not the only relevant information. But when the length of the training period is negligible, or when the expected length of time of employment is affected by other factors - as when there is a 
high turnover of employees independently of health concerns -- the information from genetic testing is irrelevant to qualifications. Moreover, even when a genetic test might provide information about the probable length of a person's career, it would be wrong and foolish to rely on this information alone (unless the expected curtailment was exceptionally dramatic), neglecting the other criteria -- aptitude, reliability, cooperativeness - on which employers usually draw in making their decisions. If someone has a $30 \%$ chance of contracting a debilitating disease within the next 30 years, as opposed to the $3 \%$ chance characteristic of the general population, it would be reasonable to rank that person lower than someone with the same academic or practical qualifications and similar demeanor. Yet it would be an injustice to treat the person as less well qualified than another candidate whose record was less impressive and whose employment history raised questions about character traits. Moreover, given the uncertainties that attend the course of employment, even relatively large differences in risks (30\% vs. $3 \%$ ) can be offset by relatively small differences in other factors, even when there is a lengthy training period that demands the employer's investment.

5. Genetic testing for general risks can occasionally be justified in assessing the qualifications of applicants. Employers have the right to take into account the expected length of an applicant's career, especially in circumstances that demand a heavy initial investment in training. But the risks must always be considered in the context both of the normal turnover of employees, and the candidate's other qualifications.

In accordance with Conclusion 3, it would be expected that the use of genetic tests for general risks on existing employees would face more stringent conditions. This expectation is upheld when we consider more closely what might justify the use of such tests. If the concern is to avoid loss of productivity by firing an employee, and the loss of productivity would come about through the curtailment of a career, then the case reduces to absurdity: a situation in which an employee's career is cut short can 
hardly be remedied by firing that employee now. If the loss of productivity comes about because the employee is already working at a lower rate, there is no need for genetic testing: if the decision is just, it is just because employers are entitled to release employees who can no longer work at adequate speeds. If the loss of productivity is threatened because the genetic condition has a chance of producing lowered rates of productivity in the future, the employer gains nothing by anticipating that future and firing the employee at once. The most plausible case for qualifying these conclusions comes from recognizing that a genetic test might become available between the time that a person had been admitted to a training program and the time at which that person began to yield a return on the employer's investment. Perhaps here it would be to the employer's advantage to cut the losses so far incurred, and begin training another person. But it is important to remember both that the person bears no responsibility for any genetic condition, and that the initial hiring creates obligations on the part of the employer. Surely employers must allow for the possibility that unforeseen disasters may befall those in whom they have invested resources. It seems reasonable to assimilate the use of new genetic tests on trainees to our standard recognition of bad luck that may affect an employer, and to promote a simple ban on the use of tests for general risks on existing employees.

6. Genetic tests should not be used to determine the general health and disability risks of those already employed.

We can best approach the issues surrounding genetic tests for workplace specific risks by recognizing that, here, employers may be motivated by any (or all) of the considerations adduced above (concern for the employee's health, concern for losses of productivity, concern to avoid clearing up the workplace). It is also appropriate to ask why the employee wishes to be employed in an environment that places him at higher risk of ill-health and disability. There are two answers to this question: most obviously, employees may be driven to continue to work in environments that are 
unhealthy for them because they have no alternatives; more subtly, they may assess the risks differently from employers (Nelkin and Tancredi 1989, Draper 1991).

There are pure cases at two poles. One extreme is found when, at a cost that could be easily borne, employers can modify the workplace environment so that the risks are reduced in a way that makes genetic differences no longer relevant. At the opposite extreme are situations in which employees have equally good employment options in environments that do not increase their risks of disease. Only irresponsible or ignorant employers fail to clean up the workplace when it is economically feasible for them to do so; only perverse or misguided employees choose jobs that increase their risks of disease and disability when equally good alternatives are available.

Focusing first, as before, on the evaluation of new applicants for positions, it is relatively easy to conclude that use of a genetic test might be justified if an employer had no feasible way of modifying the workplace environment and if there were excellent alternatives available to those people who are sensitive to the substances released in the workplace, and, moreover, that clear-headed applicants ought to welcome the use of such tests. Further, use of genetic tests would be quite unjustifiable if it were simply to serve as a means for avoiding the manageable costs of modifying the workplace. The troublesome cases are those in which no known, economically feasible, way of modifying the workplace exists and in which the applicants are bereft of other job opportunities. There are surely very many cases like this in the USA, especially in those areas of the country in which there is effectively one employer for a local population. Even at an early stage of adulthood, people often have overwhelming reasons to remain in a particular place - their entire system of social and family support, needed to make their lives work, might break down if they were to leave - and if their opportunities for employment are limited to a factory or plant environment containing substances which are toxic to people with their genotype, then they are caught in a cruel bind. To release them from that bind, it is necessary to 
create new job opportunities for them, perhaps by admitting them to retraining programs. Especially in places in which employers benefit from the existence of a captive workforce, it is reasonable to demand that a significant portion of such retraining and job replacement programs should be borne by the employers.

7. Employers have the duty to modify the workplace environment, when they can do so in an economically feasible way. Applicants have no right to protest genetic testing when that testing is for workplace specific risks, and when they have equally good employment opportunities in environments that do not pose a threat to them. When the environment is unmodifiable, conflicts between employers and applicants over the use of genetic tests can - at least sometimes - be settled through retraining programs and job creation programs. Employers who benefit from the absence of competition for their employees, and who use genetic tests to distinguish among applicants, have the responsibility to contribute significantly to the costs of such programs.

In light of Conclusion 7, employers have a choice: they can either decide to use the genetic tests to avoid losses of productivity, and pay compensation by contributing to the employment of those whom they refuse to hire on the basis of the tests; or they can decide not to use the tests, leaving the applicants free to decide if they want to be tested for the genetic susceptibility, and if they test positive, if they want to take the risks posed by the workplace.

Many real-life situations are, of course, far less clear-cut than has been suggested here: how to interpret the notions of "feasible cost" and "equivalent opportunities" will have to be decided in particular concrete cases. Other complications will be addressed shortly. First, however, it is important to consider how the conclusions about testing for workplace specific risks are affected when we switch from applicants to employees. In accordance with Conclusion 3, the 
obligations of employers towards employees are increased. Thus, if an employer should decide to use genetic testing to assess the workplace specific risks of existing employees, it is appropriate to demand that the employer take extra steps to ensure that the transition from the hazardous workplace to a safer environment can be managed as easily as possible. This might involve severance pay, greater contributions to training programs, the transfer of the employee to a different work environment at equivalent pay, or any number of other compensations.

8. Use of genetic testing for workplace specific risks, when the tests are administered to employees, is governed by Conclusions 7 and 3. Thus the obligations of the employer delineated in 7 are increased, and are increased proportionally to the employee's previous service.

In many circumstances, as already acknowledged, the sharp distinctions that have figured in the discussion so far do not apply. Many environments in which toxic substances are present could be altered to a greater or lesser extent (Draper 1991 4649). These changes might reduce the risks for all workers and effectively abolish the difference between the bearers of rival genotypes, or they might continue to show a significantly greater risk for the bearers of one genotype. There are a number of possibilities, some of which are depicted in Figure 5.1. In any situation in which there are known ways of reducing the toxic substance in the workplace environment, by varying amounts with varying costs, part of the analysis can profitably be conducted by considering graphs of the kinds shown in (a)-(d), and juxtaposing them with graphs of the kind shown in (e) and (f). In case (a), all employees are currently at high risk for disease, with the bearers of genotype A being at greater risk than those of genotype B. If the costs of reducing the level of the toxic substance are represented by (e), then both levels are significantly reduced, and, although A-bearers are still at greater risk than Bbearers, the differences are now diminished. Assuming that employees and potential employees currently have no equivalent alternatives, it seems reasonable to demand that 
the employer reduce the level of the toxic substance to that corresponding to the feasible cost, and not to use genetic tests to distinguish between A-bearers and Bbearers. By contrast, if the dependence of risks on toxin levels are as represented in (b), and the schedule of costs is either that depicted in (e) or that shown in (f), nothing the employer can do will eradicate the difference in risks between A-bearers and Bbearers. Here, it would seem, genetic tests are appropriate, but, in accordance with the demands of Conclusions 7 and 8 , they should be accompanied by a commitment to job programs for the A-bearers. If (c) is combined with (e), it is possible to modify the environment in a way that makes genetic testing irrelevant: the differences in risk between A-bearers and B-bearers are eliminated. On the other hand, if (d) is combined with ( $f$ ), although the risks for bearers of both genotypes are low, there is a significant difference between those of A-bearers and those of B-bearers. Under these circumstances, everything depends on the possibility of providing equivalent employment opportunities. If that can be done, genetic testing is justifiable. If it cannot, then the right of A-bearers to assume the risks of the workplace should be acknowledged.

Another complicating factor in real-world situations is the idea of "equivalent forms of employment". Many workers in plants that pose health hazards have some other opportunities for employment. Typically, they choose to risk their health because they can maintain themselves and their families at a higher standard of living by taking jobs in hazardous environments. To demand that they should receive the opportunity to work at exactly the same rate of pay that they now receive is unrealistic. To accept the likelihood that their wages will be so far reduced that their way of life will have to change completely is surely too harsh. Job replacement and retraining programs must allow for a lowering of real wages without making the bargain so unattractive that workers are forced to sacrifice their health. Genetic testing, impartially applied, might expose how cruel are the constraints on many workers in the 
USA. The goal of a policy for permitting genetic tests in the workplace should make it possible for employees to avoid courses of action which are predictably hazardous to their health.

The cases that will confront any regulatory policy are too various to admit of simple algorithms, and it would be folly to pretend that the Conclusions assembled above suffice to resolve them. Nonetheless, it seems worth proposing, tentatively, that genetic testing in the workplace be subject to a commission, which would hear employee complaints and would award compensation if employers had used genetic tests in violation of Conclusions 1-8. In resolving the diversity of complex cases, it would be necessary for the commission to take into account the kinds of facts represented in Figure 5.1, as well as the possibility of providing alternative forms of employment for those affected by the tests.

Three further points deserve discussion. First, if genetic tests are to be used to assess susceptibility to disease in the presence of toxic substances, it is surely important that such tests be made available to potential employees before they have made decisions that commit them to employment that involves exposure to those substances. If, for example, a factory is the main employer in a town, if its manufacturing process involves the release of chemicals that place individuals with particular genotypes at high risk, if no way is known to lower the frequency of emission of those chemicals, and, consequently, if genetic tests are used to assess applicants for jobs, then it is important that those tests should be administered while children are still in school, so that they can take steps to find alternative forms of work. It would be unrealistic to assume that all conflicts will be avoided in this way, but early testing will surely allow many people to make more satisfactory plans.

Second, it may seem that there is a short argument for rejecting the use of genetic tests in employment situations, on the grounds that employers ought not to use statistical information about groups and that the results of genetic tests are, in most 
cases, probabilistic in character. However, a blanket condemnation of appeals to statistics would undermine employment practices that seem perfectly justifiable. In evaluating candidates, probabilistic judgments are everywhere: in administering the proficiency tests that we regard as paradigmatic of fair employment practices, employers frequently rely on a correlation between test results and performance in the job that is to be assigned. Some probabilistic assessments are surely objectionable. Assembling statistics on the basis of racial, ethnic, and sexual differences is often suspect, and sometimes clearly unjustified, because the frequencies recorded are biased by the past history of opportunities afforded to the groups in question. Precisely because genotypes have traditionally not been recognized, they cannot have served as the direct target of earlier discrimination, and, hence, except insofar as they correlate with phenotypic characters on which discrimination has been practised, the chances associated with genotypes are legitimately invoked to assess qualifications. The previous discussion makes clear the ways in which appeal to those statistics must be balanced against other factors in employment decisions, but they cannot just be ruled out as inappropriate.

Finally, it may be thought that there is a very simple way of responding to the conflicts introduced by genetic testing in the workplace, to wit enlarging the scope of the Americans with Disabilities Act (ADA). The trouble with this proposal is that, if the pool of jobs available to those classified as disabled is not expanded, people with genetic predispositions to disease will be competing with people with current disabilities for the positions originally earmarked for the latter group. One effect of this will surely be to dilute the benefits which the ADA has brought to those whom it was intended to serve. If, on the other hand, the pool of jobs for the disabled -- now understood more broadly - is to be expanded, then some principles will be needed to guide the expansion, and many of the same issues discussed above will have to be 
resolved. In particular, it will be necessary to decide when a person with a workplace specific genetic susceptibility has the right to work in the hazardous environment.

Ideally, workers would not have to choose between sacrificing their health and maintaining their families, and genetic tests would be welcomed as means of steering them towards forms of employment that would provide economic security without undue hazard. Blanket permission to workers to avoid genetic testing is a natural response to the idea that employers will use genetic tests to avoid cleaning up the workplace. The interests of workers are best served by insisting that environmental modifications be undertaken where it is feasible to do so, that genetic testing be accompanied by the creation of job opportunities, and that, instead of perpetuating choices between health and wealth, we try to take steps towards the obvious ideal.

\subsection{Social Stigmatization}

The most obvious ways in which genetic information about individuals can be used to their detriment are in the contexts of obtaining insurance and employment, but, even if these problems were addressed in the ways suggested in $\$ \$ 5.4-5.6$, there is a residual worry that those who bear certain types of genotypes will be stigmatized, so that genetic testing will create a new class of social pariahs. It is important to consider this worry, because it underlies much opposition to the introduction of genetic testing.

At first glance, it would seem extremely unreasonable to adopt attitudes of disdain towards those who carry particular alleles: for this means of classifying people brings with it the knowledge that they are in no way responsible for the property that is the basis of the classification. Members of groups currently stigmatized -- such as homosexuals -- have sometimes welcomed the suggestion that the attributes that provoke distaste or scorn in others (e.g. being homosexual) have a genetic basis, on the grounds that, if this were known, the prevalent social attitudes would show greater understanding and sympathy. Although this hope is probably too optimistic, it does 
acknowledge a widely-shared predilection not to blame people for conditions for which they are not responsible.

Why then should there be fear that if information about genotypes becomes known, it will issue in social stigmatization? Learning that someone carries a set of alleles that predispose him/her towards a disease (colon cancer, diabetes, Huntington's disease) would seem more likely to prompt a reaction of sympathy rather than one of dislike, contempt, or revulsion. (Although it should be noted that the sympathy of others is often felt as an additional burden.) Concern is surely generated by the possibility either that genetic susceptibilities to disease will become associated with other, social, traits that are stigmatized, and that the stigma will become attached to the bearers of the pertinent genotypes, or that genetic information for behavioral propensities that are currently viewed with scom or repugnance will lead to the stigmatization of bearers of alleles, irrespective of their actual behavior.

The most likely ways in which the bearers of disease genes will become vulnerable to social stigmatization involve some prior form of discrimination against those individuals. If, for example, genetic tests are introduced and people at high risk for various diseases become uninsurable and unemployable, then there will indeed be a new class with many of the attributes that cause stigmatization: people in this class will be poor, they will not work, deprived of other opportunities they may come to have a higher crime rate, and so forth. In addition to the burdens imposed by their genetic constitution, these people will have to bear the burdens of inadequate medical care, unemployment, and, as a consequence, social stigmatization. This last burden will be a genuine further problem, but it is not the consequence of their genetic condition alone, and can be eliminated by taking steps to ensure that genetic tests are not used to close off the avenues through which individuals earn the respect of others. The threat of social stigmatization thus underscores the need for attention to the problems of insurance and workplace discrimination that have been considered in $\$ \S 5.4-5.6$. 
Solutions to those problems can be expected to foster a social environment in which the genetically unfortunate receive the respect -- and sympathy -- they deserve.

The issues surrounding the possibility that genetic information about individuals will lead to the identification of springs of behavior, and, consequently, to stigmatization of people who have genotypes that dispose them to forms of behavior that are frowned upon, involve a range of issues, some of which have been touched upon in section 3 and which are treated at greater length in section 8 . As noted in section 3, the primary information that will be generated will concern those human traits and propensities which are widely shared with other organisms. Although there is widespread publicity for claims about genes for behavioral traits -- alcoholism, aggressive behavior, homosexuality - it is crucial to recognize that these claims are remarkably difficult to substantiate. What molecular biology is very good at is identifying those alleles that have large effects on the phenotype, and which are similar (more exactly, homologous, similar by descent) to alleles that are expressed in nonhuman organisms. The exciting traits that attract popular speculation do not meet these conditions, and justification of claims about them typically rests upon studies of behavior genetics with its notoriously indirect (and, frequently, inconclusive) methods. If the Informational Precondition (Conclusion 2) is honored, then genetic information will not be employed to reach conclusions about people's behavioral propensities.

However, this is far too idealistic. Because of the fascination that certain types of human behavior have for us, there is little doubt that claims about aggression and sexual preference (to cite two prominent examples) will continue to be made (Lewontin, Rose, and Kamin 1984, Hubbard and Wald 1993). The past decades have witnessed a number of occasions on which claims about the genetic basis of such traits (and about genetic explanations for differences in traits) have been widely broadcast, sometimes with adverse social effects. So, for example, it has been proposed that 
there are genetic bases for differences in intelligence among members of different races, that there are genetic differences between men and women that account for stereotypical sex roles and for difference in intellectual aptitudes, and that there is a genetic basis for sexual orientation. Some of these claims have been thoroughly discredited, others remain controversial. It would be tragic if the release of genetic information caused people to be stigmatized because of loose associations between combinations of alleles and forms of behavior that prompt hostile reactions.

However, the sources of the problem here do not lie in the information about individuals. One trouble stems from willingness to accept claims about genetic bases for behavior on evidence that would be regarded as inadequate (sometimes ludicrously inadequate) in standard scientific contexts. The second is the consequence of deep failures to respect -- or even tolerate -- those who are different in various respects. Even if claims about a genetic basis for (say) homosexuality were correct, it would be morally unjustifiable to discriminate, in action or in attitude, against those who bear the relevant genes. (The Nazi programs in which Jews, Gypsies, and homosexuals were sterilized or murdered would not have been morally defensible, even if the claims of "race biology" had not been so desperately wrong.)

Opponents of genetic testing (e.g. Hubbard and Wald) are quite right to see that release of genetic information in a society that refuses equal respect to some of its citizens and that has a credulous fondness for thinly supported claims of genotypephenotype association is potentially dangerous. If we are not to be forced to choose between achieving the genuine benefits from genetic information and avoiding the threat of stigmatization, it will be necessary to take very seriously the Informational Precondition and to continue to work towards a more tolerant society. Public advocacy of measures that assure equal rights and equal protection to those who have frequently been targets of social discrimination can play a large role here. It is also worth remembering that, in the absence of efforts to promote greater tolerance, those 
who can be identified as belonging to particular groups on non-genetic grounds will continue to be vulnerable to discrimination. We should think of genetic testing as exacerbating a problem of discrimination that ought to be addressed in any case.

There is a more pragmatic way to approach the particular difficulties introduced by genetic testing. Given that insurers and employers are restrained from using genetic information, motivation to acquire and spread such information will surely be reduced. As noted in $\$ 5.1$, this may make it easier to safeguard sensitive information about individuals. Informational databases will surely always be vulnerable to those with sufficient resources and ingenuity to crack them, but, if the information cannot be used by insurers and can be used only in circumscribed ways by employers, the two main channels through which data about the alleged "behavioral genotype" of a subject might pass into general circulation will be blocked. People may still be subject to rumor and innuendo -- in the ways that they are now -- and malicious or careless people who have access to genetic profiles may spread gossip. But to conjure up a vision of inquisitive hackers determined to glean from databases facts about the genotypes of others may be too fearful. Provided that there is no motivation for insurers and employers to acquire genetic results that can then circulate more widely, the extent to which ascriptions of allelic combinations can be made with any appearance of plausibility is likely to be limited.

\subsection{Reinforcing Prejudice}

The final topic, the possibility that genetic information will reinforce existing prejudices against the members of certain groups, overlaps with the discussion of $\$ 5.7$. Episodes from the recent history of genetics and physiology reveal that the threat is real. The exclusion of those with sickle-cell trait (overwhelmingly blacks) from the Air Force Academy, the contrast between the administration of the (botched) program in sickle-cell testing and the (successful) programs of Tay-Sachs testing (OTA 1990), 
and the policy pursued by American Cyanamid (which debarred female employees of childbearing age from working with certain toxic substances), all show that the ways in which genetic information is used reflect prevalent stereotypes and prejudices (Duster 1990, Andrews et.al. 1994, Draper 1991 68-76, 83). The gross failures to respect the Informational Precondition in the first and last case - bearers of sickle-cell trait are not more vulnerable to collapse at high altitudes, male employees are also at reproductive risk from toxic substances -- are surely traceable to antecedent judgments about the abilities of blacks and the proper roles of women respectively. False conclusions were readily accepted because they fitted so well with entrenched ideas (P. King 1992, Nelkin and Tancredi 1989 104-5).

Full analysis of the human genome might serve as a corrective to some prejudices. Different ethnic and racial groups are particularly susceptible to different genetic conditions. Thus the highest frequency of the sickle-cell allele is among American blacks, whites of Northern European extraction are at greatest risk for CF, Ashkenazi Jews have the highest probability of carrying a Tay-Sachs allele, people from the countries that ring the Mediterranean are the most likely to carry genes for the various forms of thalassemia. Detailed work in human molecular genetics might reveal that the catalog of known genetic disabilities is distributed across racial and ethnic groups in roughly even fashion, engendering conviction that while we are genetically different all groups are "genetically equal" (or, perhaps, "equally defective"). Although this would be a welcome consequence, the fundamental moral point is quite independent. However the genetic lottery has distributed the fortunate and unfortunate holdings, all people are worthy of equal respect, and it would be quite wrong to suppose that inequality in the distribution represented gradations of superiority and inferiority among races.

Those who are charged with the task of securing the equal rights of groups that have historically been disadvantaged -- women, blacks, Hispanics, and others -- should 
be vigilant to the possibility that genetic testing, allegedly based on attention to qualifications, will use genotypes as code for racial features. Employers may protest that they are hiring those best qualified, that their decisions to use genetic information are based on the considerations acknowledged in \$5.6. But when genetic conditions are correlated with racial characteristics (or with the characteristics of other groups that have suffered discrimination in the past), it is crucial that the claims to relevance be closely scrutinized. Offices that attempt to promote equal opportunity must be thoroughly informed, both about the general possibility of cloaking prejudice in scientific claims about genotypes, and about the particular kinds of correlations that human molecular genetics has disclosed. 


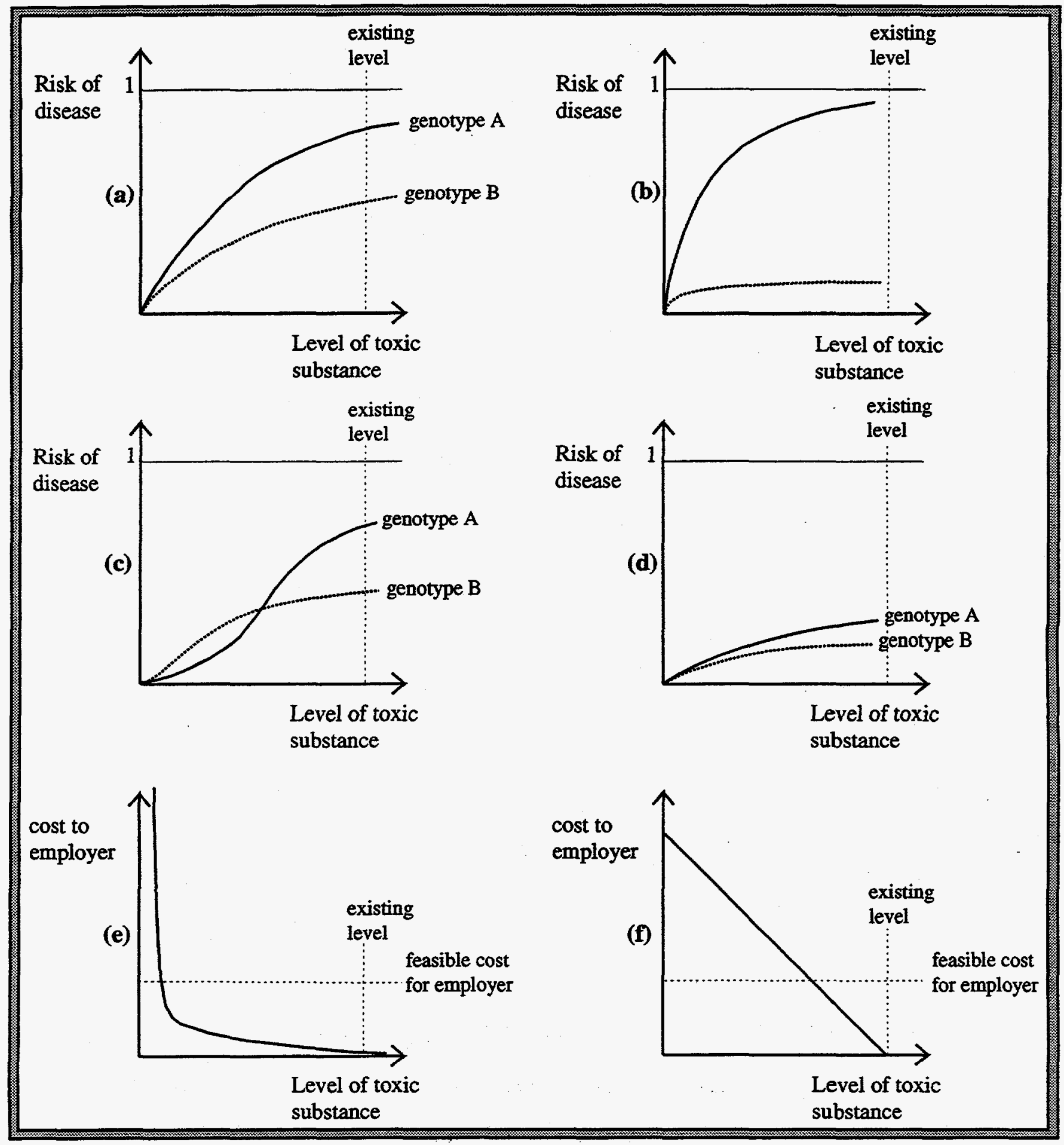

Fig. 5.1 


\section{Prospects for Therapy}

\subsection{Multi-levelled Possibilities}

As many defenders of the HGP have cogently argued, the ultimate goals are to prevent and/or treat major diseases (Caskey 1992). Developing genetic tests may play an important part in the strategies for coping with disease, but the project would have far less social value if it were merely to deliver a battery of tests. Critics point out that there is no guarantee that increased knowledge of molecular genetics will lead to new forms of therapy or new strategies of prevention (Lewontin 1992). The example of sickle-cell anemia is a sobering reminder: forty years after the molecular basis of the disease was recognized, little has been done to alleviate the plight of sufferers. What grounds are there for thinking that sickle-cell is atypical?

Sickle-cell disease occurs because the red-blood cells (erythrocytes) of people with two copies of the sickle-cell gene contain only abnormal hemoglobin (erythrocytes are, to all intents and purposes, simply sacs containing hemoglobin molecules; they have no genetic material). Under conditions of low oxygen, the abnormal hemoglobin causes them to become malformed, so that they take on a characteristic "sickle" shape (whence the name of the disease), becoming stuck in capillaries, blocking blood flow, and preventing the delivery of oxygen to muscles, limbs and organs. Precisely because the erythrocytes are such peculiar cells, without their own assembly of genes and distinctive proteins, it is very hard to deliver normal hemoglobin to them and thus to prevent the malformations. There are thus grounds for hope that the sickle-cell example is anomalous and that it does not reflect the prospects for future molecular medicine.

Plainly, it is too early to do much more than speculate, but there are already encouraging signs. As noted in section 4, there is reason to believe that the identification and sequencing of genes associated with colon cancer will translate into improved methods of early detection and disease prevention. The cases of CF and 
SCID, to be described below, also show how molecular understanding can transform the treatment of a disease. The main goal of the present discussion is not only to review these particular cases, but also to emphasize that molecular genetics holds open possibilities for intervention at a number of different levels. After developing this point, discussion will turn in $\$ 6.3$ to the moral questions that surround a particular type of intervention, gene replacement therapy.

Assume, for present purposes, that the notion of disease is well understood. A genetic disease is a disease for which there is an assemblage of alleles which, in standard environments, cause bearers of those alleles to manifest the phenotype associated with the disease. Standard environments are those in which people normally live, so that the notion of a genetic disease must always be thought of as tacitly relying on the way of life of a particular group. An abstract way to characterize treatments for diseases is to see them as modifying the standard environments, finding new ways in which people can live so as to avoid the phenotypes associated with the diseases.

The naive thought is that a genetic disease would have to be treated by modifying some of the genes. Gene modification -- more exactly, gene replacement therapy - is indeed a tactic that contemporary molecular genetics has made possible, but, as the last paragraph suggests, genetic diseases can be attacked by introducing a non-standard environment. This can be attempted at any of a number of levels. Doctors can try to change the immediate milieu in which the genes act, or try to deal with the consequences of their actions within cells, tissues, or organs, or they can try to modify the environment external to the patient. The case of PKU represents the last approach. Here it is understood that the effect of a defective gene is to interfere with the processing of phenylalanine, so that, in standard environments (those in which children are fed normally), phenylalanine builds up in the body. Modifying the 
patient's environment, by giving a low-phenylalanine, high-tyrosine, diet prevents the build-up and the phenotype associated with the disease.

Environmental interventions may have costs. In the treatment of PKU, for example, the diet is expensive, socially disruptive, and unpleasant; nonetheless, the costs are small in comparison to the gravity of the condition (mental retardation, usually severe) that is avoided. Similarly, standard treatments of beta-thalassemia give patients frequent blood transfusions, causing them to accumulate large amounts of iron. The excess iron can interfere with normal growth and development, as well as producing considerable damage to the heart and liver (Friedmann 1991 68). Modifying a patient's environment, to deal with problems caused ultimately by defective or absent gene products, may generate new problems that have to be addressed.

Between the extremes of changing the patient's external environment and replacing a gene lies a host of intermediate strategies of intervention, which alter the patient's body, the hierarchy of environments in which genes are expressed. A small sampling of the possibilities is as follows:

A. The defective gene fails to yield a protein that is needed for a reaction in a particular organ or tissue. The trouble is corrected by delivering the protein directly to the organ or tissues. (Steinberg et.al. 1993147 note that there are sometimes limitations to this method.)

B. The defective gene fails to yield a protein that is needed for some reaction that would break down a molecule in a certain type of somatic cell. The problem is addressed by delivering to cells of that type a substance that is able to break down the molecule.

C. The defective gene fails to yield a protein, needed for a reaction that would convert a molecule that is toxic in large quantities into harmless constituents. A substance is introduced that changes the biochemical pathway leading to the harmful molecule. 
D. The defective gene causes a certain type of immune cell to fail to make proper distinctions between the patient's own cells and foreign cells. The patient is treated by injecting a substance that recognizes the misbehaving cells and incapacitates them.

E. The defective gene causes a protein that fortuitously represses the transcription of a second gene. The patient is treated by delivering molecules that bind strongly to the defective protein, and thus prevent it from repressing transcription.

Some of these scenarios already have instances: the treatment of hypercholesterolemia is akin to A; the use of penicillamine to treat various disorders (such as Wilson's disease and cystinuria) is like B; various attempts to attack autoimmune disease resemble $\mathrm{D}$. But the important point is that understanding of the pathway between genotype and phenotype makes possible a variety of modes of action. Some of these would make a highly indirect use of knowledge from molecular genetics. For example, tolerance for tissues from others might be increased by making molecular interventions in the immune system, enabling organ transplants to be carried out more successfully (Parkman 1991).

It is wrong to think of genetic knowledge as completely compartmentalized. Work on the basis of a particular disease, including the sequencing of the gene, the recognition of the protein coded, study of the ways in which that protein interacts with other molecules, both in normal functioning and in producing the disease condition, may bear on other diseases as well. Strategies that work in the case of one disease may suggest relatives that could be applied in the case of another, or we may discover that a familiar method of treatment, that seems largely a failure, does not work because it effectively turns the disease condition into a problem whose solution elsewhere offers the key to success in the case at hand. The information resulting from the HGP will probably issue in a hodge-podge of methods that can be used to refine and supplement 
one another in ways that are initially unforeseeable, but which ultimately stem from the systematic search for as many human genes as possible (see section 3 and Watson et.al. 1992511 ff.).

We may expect jury-rigged techniques which are effective to different degrees in different cases. The most direct approach, for each problem, would be to replace the genetic material which, in concert with the environment, causes the trouble. The next task is to ask when this direct approach is appropriate.

\subsection{Gene Replacement - Necessity and Possibility}

When, if ever, would it be necessary to treat a patient by introducing new genetic material? The previous discussion emphasizes the number of ways in which we might try to intervene between genotype and phenotype. It might be thought that gene replacement was especially critical when a mutation occurs in an allele that is expressed early in development so that there are many causal consequences of the failure to encode normal protein. There are surely cases in which the presence of mutant alleles produces a developmental cascade, and the most terrible hereditary malformations may well be among them. But it is far from obvious what can be done for a fetus that bears any such genetic condition, even if we were confident that we had identified the causes of the phenotypic problems. Abortion provides the only remedy currently available, and, without extraordinary changes in our power to treat fetuses, that is likely to continue to be the case. Moreover, if the time at which the allele is first expressed in development is sufficiently early, then the irreversible effects of the mutant genes may be felt before it is possible to test for the condition.

When a baby is born as the result of In Vitro Fertilization (IVF), it is possible to test at the stage when the conceptus contains only a few cells, and it would be possible, in principle, to replace early acting genes. However, in such instances, the far simpler strategy of only implanting those embryos without genes for major defects 
is also available. Thus it is hard to see how gene replacement therapy could be especially relevant in this context.

It is tempting to conclude that where gene replacement therapy is most needed -in the case of early acting genes -- it is either impossible or else redundant. But this would be a mistake. Gene replacement therapy might simply be part of the arsenal of techniques used to treat conditions that we cannot remedy by higher-level interventions. Rather than trying to send a normal protein to a particular type of cell, it will sometimes be simpler to send some genetic material and have the cells manufacture their own protein.

Consonant with the pragmatic attitude of the last section, it is important to realize that the genetic material introduced need not be that for which the patient has a mutant allele (or two mutant alleles). Often what is required is to restore the ability to make a normal protein. But sometimes the task will be to inactivate a mutant protein - say by introducing a gene that codes for a protein that will bind to or break up the unwanted proteins. Or we may want to destroy certain cells completely, by introducing genes that code for cell-surface proteins that attract the attention of the immune system. The last tactic is obviously attractive in trying to deal with incipient tumors: if we could find ways to detect cells that were building a tumor, then we could try either to destroy them, or subvert their relations with other cells, or to stop them growing. Large-scale understanding of the genome and its products is likely to generate a number of obvious ways of tackling medical problems, and, perhaps, an even larger number of roundabout methods of tricking patients' bodies into an approximation to normal functioning.

When is gene replacement therapy possible? The first problem to be overcome is that of sending genetic material to the right cells. There are fundamentally two modes of delivery: one involves removing cells from the patient, inserting DNA directly into the cells in vitro (usually by microinjection), growing the modified cells in 
large numbers, and then replacing them in the patient; the other relies on the construction of a vector that can take genetic material to cells within a patient's body. (for example, appropriate pieces of genetic material are inserted into a virus that has been modified to render it harmless, the virus is equipped with the capacity to recognize the cells being targeted, and the patient is inoculated with the virus). The delivery problem is currently easier to solve using the first method, However, there are obvious limitations to the procedure, since one cannot remove so many cells from the patient that an organ or tissue becomes incapacitated. The trick is to identify and remove stem cells, those cells that give rise to large numbers of descendants of a particular type; bone marrow cells are a familiar example of stem cells. With viral vectors it is important to equip the protein viral coat with a site that preferentially binds to cells of the appropriate type. The more that is known about the molecular biology of various kinds of cells, specifically about what kinds of membrane proteins they have, the easier it will be to engineer viruses for particular roles in delivery. Frequently, an ideal solution would be to configure the virus so that once the DNA has been inserted the cell surface proteins are modified, so that the cell is no longer receptive to further viral vectors; this would avoid the danger of delivering too much DNA to some cells.

At present, delivery techniques are limited by a large number of difficulties, most notably that of sending genetic material to the brain. Because neurons do not divide, it is impossible to use standard viral vectors for delivery (since those vectors rely on the mechanisms for cell division to do their job). Researchers hope that it may be possible to remove and modify cells that give rise to neurons (basal glial cells -- see Nichols 1988 37), but, as things now stand, there are no reliable ways to deliver genetic material to the brain. Thus, while it appears feasible to engineer vectors for targeting some cells of patients with Lesch-Nyhan syndrome (for example), it is not 
now possible to alleviate the behavioral aspects of the disorder (which are its most poignant manifestations).

Sending a vector to the right cells (or inserting material into stem cells) is by no means the only problem facing gene replacement therapy. Once the DNA has been delivered to the cell, the next step is to insert it in the right place into the genome. Currently, there is no way to control the point of insertion. One potential problem is that the DNA might integrate into the middle of a functional gene, thus disrupting some important aspect of cellular functioning. (If coding regions occupy about $5 \%$ of the genome, and $80 \%$ of inserts within coding regions would cause problems, then about $4 \%$ of the cells reached would be disabled in this way. But this is probably an underestimate because it does not take into account the possibility of interfering with regulatory sequences.) A second worry is that, if the DNA is delivered without any control regions, then, even if it lands in the intergenic stretches between coding regions, nothing will be gained because there will be no way to switch the gene on. This difficulty can be overcome by equipping the protein-coding part of the DNA insert with its own control system, but this requires understanding enough about systems of control within the cell to make an educated guess about which regulatory sequences will not interfere with the cell's ways of regulating other genes.

The hardest problem of all is that of controlling gene expression in time. Ironically, with some very severe conditions the problem can be overcome because a gene needs to be producing protein all the time. Severe combined immune deficiency (SCID) is like this: the disease results from the presence of two copies of a mutant allele with the result that a protein needed permanently is not supplied. SCID is currently being tackled by gene replacement therapy involving the delivery of DNA segments coding for the crucial protein, equipped with a regulatory mechanism which ensures permanent expression. Moreover, if the aim is to insert DNA to kill a cell (as in the earlier speculations about possible ways of coping with incipient tumors), niceties 
of timing are irrelevant. However, for the vast majority of genetic problems, shooting some DNA somewhere inside a cell and having it constantly churn out protein is the molecular equivalent of hitting the body with a mallet. The timing of expression needs to be set according to the cell's needs, and this requires understanding how to devise a regulatory system that will be sensitive to intercellular systems. Once again, the systematic approach to the whole genome that the HGP adopts may be important in remedying ignorance here.

Gene replacement therapy is currently in its infancy. Besides the treatment of SCID, there have been recent successes, based on the sequencing of the CF gene, in treating CF patients by using an aerosol spray to transport retroviruses directly to the lining of the lungs. To underscore the pragmatic themes of the approach adopted here, there is no reason to think that there will be general breakthroughs that will solve delivery and timing problems in all instances, but it does seem likely that further research will generate a motley of techniques, of various levels of generality, to transcend current limitations in an unpredictable array of cases.

\subsection{Ethical questions of gene replacement}

At present, there appears to be a consensus that gene replacement therapy is morally unproblematic when the genetic material replaced is in somatic cells and when the aim is to cure a disease, and that serious moral questions arise about using gene replacement therapy to enhance characteristics. Gene replacement therapy has already been partially successful in treating SCID, and the manufacture of an aerosol to transport appropriately modified viruses to the lungs has begun to bring relief to $\mathrm{CF}$ patients. These treatments seem unproblematic. Why?

Both forms of gene replacement therapy are directed at the somatic cells, not the germ line, and both aim to restore health, not to enhance the patient's abilities. Nevertheless, it is worth probing the suggestion that these are the pertinent moral 
distinctions. Since gene replacement therapy is in its infancy, it is impossible to appeal to actual examples of treatments that have been proposed. Instead, it is necessary to consider some hypothetical cases with an eye to recognizing what features would make alteration of genetic material permissible or impermissible.

One morally relevant consideration concerns whether or not we are changing the identity of the person in whom the genetic replacement is made. It is possible to imagine that some forms of gene replacement would so alter the neural chemistry that the person's personality would be enormously different. Sometimes, this might be seen as liberating: imagine that the problems of delivery to neural cells are solved, and that it becomes possible to replace the allele that causes Lesch-Nyhan syndrome. On other occasions, a strategy for tackling some neurodegenerative disease might produce enormous changes in mood and emotional response: someone who is contemplative and morose might become thoughtless and permanently cheerful. The issues that would arise in such cases are akin to those that figure in discussions of electroconvulsive therapy and frontal lobotomy, and those issues reveal that the proposal that gene replacement therapy to tackle disease is always permissible if the replacement is made in somatic cells is too simple. An extra condition must be met: the identity of the patient must be preserved.

Another obviously relevant feature (one highlighted in many discussions of germline gene replacement therapy) is the state of knowledge of potential side effects (Wivel and Walters 1993, Suzuki and Knudtson 1989). It is hardly ever possible to have full understanding of the range of consequences that flow from a medical intervention. The crucial question concerns when gene replacement therapy brings greater levels of uncertainty than in those medical interventions that are usually counted as unproblematic (despite the risks that attend them).

Claims about the safety of gene replacement will surely be based on experiments with nonhuman animals. The cases can be divided into two types: (a) those for which 
there is an animal model in which a similar phenotypic condition is produced by a homologous gene (or combination of alleles); (b) those for which the only existing animal models don't manifest the phenotype or produce it in a very different way. Other things being equal, it appears that examples of kind (a) will provide a firmer basis for predicting the effects of gene replacement in human beings -- although it is important always to be aware that introduction of new genetic material may have quite different effects because of the different genetic background. When the condition to be treated has a behavioral syndrome among its constituent features, the medical predicament will usually be of kind (b), and there will typically be greater uncertainty.

At present our inability to control the place in the genome where new DNA will be inserted is an obvious source of unpredictability. In some cases, this may be reflected in so much variation among the phenotypes of experimental animals that we have little basis for judging what the effects on human beings would be. Yet even when it is uncertain whether or not the treatment will prove beneficial, provided that gene replacement therapy never seriously worsens the condition of the animal, the risks will sometimes be worth taking, precisely because the disease being targeted is so ravaging. Consider a possible future gene therapy for Lesch-Nyhan syndrome. Initially, at least, this would probably involve taking some chances with patients who might suffer unforeseen damaging effects, including effects on their brains that are quite undetectable in experimental animals. Given the plight of those patients if we do not intervene, we may well have grounds for thinking that the risks are worth taking. (The decision to use gene replacement therapy to attack SCID seems to rely on a similar attitude towards the risks and the consequences.)

If, however, the techniques for inserting DNA into cells become much more exact, then the distinction between cases of type (a) and those of type (b) may become irrelevant. Suppose that it becomes possible to remove from a genome any (genesized) segment of DNA and replace it with a chosen DNA segment, and that this 
general ability is well-supported by experiments on nonhuman organisms. On this basis, it will become reasonable to believe that DNA segments in human beings can be replaced by other segments, and that a person with an allele that causes a disease can receive a combination of alleles at the pertinent locus that is typical of unaffected members of the population. The effects of that replacement will not be predictable from studies of nonhuman animals, but from our knowledge of genotype-phenotype relations in human beings. Because of the possibility of unprecedented interactions among genes, there is a very small probability of unanticipated side effects, but this risk would be very low in comparison with those standardly tolerated in medical situations.

A major worry about germline gene therapy has been the possibility of unforeseen consequences (Wivel and Walters 1993, Suzuki and Knudtson 1989). The previous discussion demonstrates that the distinction between germline and soma is not the crucial one for the appraisal of risks. As things now stand, there are large risks involved in many forms of somatic gene replacement therapy because of the inability to target the insertion and regulate the expression of the new DNA. That is not to say that those risks are not worth taking, for gene replacement therapy may be the only available way of treating a severe condition and, for some conditions, the niceties of insertion and timing may be dispensable. If gene replacement therapy becomes highly precise -- that is, if cells can be targeted and new DNA inserted in place of the mutant stretches (bringing the new DNA under the control of those sequences that would normally regulate the expression of the gene, and thus, presumably, solving the problems of timing) -- then there is no more reason to worry about unforeseen consequences in the case of germline replacement than there is in the case of somatic replacement. In effect, the genome of the individual treated is modified to become equivalent, at this locus, to that of normal members of the population, and there is only a minute probability, based on idiosyncratic interactions between the normal alleles at 
the modified locus and the rest of the patient's genome, that there will be unforeseen damage either to the patient or to descendants.

An apparent advantage of germline gene replacement therapy is that it will eliminate the need for further interventions on offspring. Under the ideal circumstances of technical improvement envisaged above, this is indeed the case. Somatic gene replacement for CF (say in the lungs) might alleviate the condition of a patient, but we would, apparently, have to repeat the process on (some of) the patient's children (assuming that the person married another CF carrier); in the case of Huntington's Disease, we would expect half the children to carry the disease gene, and these children would have to receive somatic gene replacement therapy; so it would go through the generations. If, however, we could intervene precisely at some stage, replacing the disease causing allele in all cells, including germ cells, then the descendants would be freed from the disease and from the need for somatic therapy. Assuming that some risks are involved in any operation, germline gene therapy might thus appear preferable.

It must be noted, though, that this scenario depends on the ability to target DNA insertions precisely. If all that can be done is to insert a desired segment of DNA somewhere within a cell, then the insertion into the germ cells will certainly be retained in the next generation, but will only be transmitted further with about $50 \%$ probability. The reason is that if the insert lies on a different chromosome (or sufficiently far on the same chromosome) from the disease locus, then the transmission of the new DNA and of the disease allele are effectively independent: under these circumstances, only half those who receive the disease allele will also bear the new, corrective, DNA.

It might be thought that the possibility of snipping out unwanted DNA and replacing it with a specific sequence in just the desired position is too fanciful to deserve attention. However, even though so precise a technique is far beyond current possibilities, it is worth recalling that it is not conceptually impossible. Current uses 
of recombinant DNA turn on our ability to make use of the ways in which enzymes preferentially recognize certain sequences and on quite exact methods of cutting and joining DNA molecules, and it does not seem impossible that these could be deployed, in clever ways, to snip out an unwanted piece of DNA, to replace it with the segment desired, and break down the DNA excised. Furthermore, there are special cases in which the problem seems much simpler. Consider those conditions -- including Fragile X Syndrome, Myotonic Dystrophy, and Huntington's Disease - in which the trouble is caused by the existence of long repeats. Here the problem is not to insert DNA but, apparently, to reduce the length of the repeat by cutting out a long stretch of trinucleotide segments. Perhaps this could be done by binding to the patient's DNA molecules that protect a small number of trinucleotides (enough to ensure that the repeat remains within the normal range) and exposing the intermediate stretch to a splicing enzyme.

These speculations are intended only to suggest that the more exact techniques on which the feasibility of germline gene replacement therapy would depend are not out of the question. Whether they are realized or not, one important consideration for gene replacement therapy does turn on questions of risk. The argument developed above undermines any simple decision-making based on the idea that somatic replacements are permissible and germline replacements are not: in all instances, the issues of risk should be confronted directly. Sometimes, the result will be that a somatic intervention is counted as impermissible; if techniques become sufficiently sophisticated, germline interventions could be justified on these grounds.

But there are other important considerations. As a number of writers have maintained, in opposing germline gene therapy, future people will be affected by decisions to replace the DNA in a patient's germline. However, future people are affected by current decisions whether or not prospective parents choose to intervene. The actions that prospective parents and their doctors perform affect the characteristics 
of progeny -- typically mothers do the best they can, in the light of the available medical knowledge, to ensure the health of their babies. Sometimes the information on which they base their decisions is incorrect and the offspring suffer in consequence. Although the children have in no sense consented to the actions, and although they may be harmed by what has been done, the parents' interventions may nonetheless be morally justified in virtue of a responsible and concerned effort to promote their wellbeing. Pregnant women sometimes have to spend the last weeks of pregnancy in hospitals, and they sometimes have to take medicines all of whose effects on the fetus are not thoroughly known. To the extent that parents and doctors in these situations can be seen, retrospectively, as doing the best they can, in the light of the information available to them, to bring healthy children into being, they cannot be said to do wrong because the children were not consulted.

Perhaps the case of germline gene therapy may be thought to have special characteristics, that it would be more cautious for parents to undergo somatic replacement therapy, waiting to intervene and replace problematic segments of DNA after the baby is born. By replacing the DNA in the germline, we may also hope to avoid damaging effects that occur while the fetus is in utero, as well as eliminating the need for future somatic modification. It may become possible, in the future, to do either somatic or germline replacement therapy on Tay-Sachs or Lesch-Nyhan patients. Assuming that the considerations of risk do not tell against germline therapy, it would apparently be wrong to oppose such therapy on the grounds that the children brought into being have not consented to the procedure: after all, if they are to be helped by either form of therapy, actions will have to be taken before they can give their consent. If we suppose further that somatic replacement would offset the main effects of the genetic condition while doing nothing to remedy the impacts of the disease alleles in early ontogeny, then the case for germline intervention is strengthened further. Moreover, in response to the frequently-voiced worry (Suzuki and Knudtson 1989) that 
germline intervention might produce irreversible effects, it should be noted that, if gene replacement techniques become sufficiently precise -- precise enough to override concerns about risk -- then we can expect that whatever could be done could also be undone. Our descendants would therefore be able to draw on just the techniques that had been used on their behalf to reverse effects of those techniques which they opposed.

Concentrating on examples like Lesch-Nyhan and Tay-Sachs helps make clear that the distinction between somatic replacement therapy and germline replacement therapy does not carry the moral weight traditionally assigned to it. Germline interventions appear frightening because of the threat that we shall not stop with amending genetic conditions which, left untreated, produce terrible suffering or severe limitations, but that we shall proceed to "design babies", inserting genes for all kinds of aesthetic traits, as well as for behavioral propensities that we want to encourage. Several points must be appreciated if this deeply worrying issues is to be properly understood.

First, the foreseeable future of gene replacement will be governed by our ability to identify alleles and their effects on the phenotype. This is most straightforwardly and reliably done for those alleles which, in mutant form, produce large, damaging, effects on the phenotype, in large measure independently of the rest of the genome and of the environment. While our techniques for delivering genetic material and regulating its expression remain crude, these are also the conditions that will lend themselves to gene replacement therapy, for it will be worth using even imperfect solutions to tackle devastating problems. If fears about engineering people with enhanced capacities are to prove justified, then it will be necessary to make advances both in identifying genes and their functions and in precise methods of gene replacement. 
Any discussion of "Frankenstein" scenarios should be based on the clear recognition that those scenarios will only become realistic options against the background of far greater knowledge than we now possess. Nevertheless, although the problem of thinking about the morally permissible limits of gene replacement in a context of greatly increased knowledge is not an urgent one, it is worth engaging in some advance discussion, if only because the steps that we take now, guided by much more rudimentary abilities to manipulate the genome, may seem to set us on a path that will inevitably lead to dreadful consequences.

One way of expressing this fear is to charge that when gene replacement therapy is employed either to make changes in the germline or to try to enhance human capacities, there is already a commitment to eugenics. The many-faceted notion of eugenics will briefly be discussed in section 8 . For the present, it suffices to recognize the difference between programs that leave reproductive decisions to the individuals concerned (the prospective parents) and those that involve standards set by impersonal authorities. Assuming that individual reproductive freedom is assured, the crucial question concerns the possibility of distinguishing morally permissible and morally impermissible ways of modifying the genome.

The question can usefully be divided into a number of subsidiary issues:

Are there some sorts of gene replacements that people ought not to decide for themselves to undergo?

Are there some sorts of gene replacements that biomedical researchers ought not to make possible?

Are there some sorts of gene replacements that parents ought not to choose for their infant children?

Are there some sorts of gene replacements that should not be conducted with respect to a fertilized egg? 
There is a strong inclination to believe that people have the right to do what they like to their own bodies provided that their actions do not affect the well-being of others. This would seem to support an individual's right to choose any type of somatic therapy, and to debar germline therapy. However, as argued above, not replacing alleles also can have harmful effects on progeny and interventions that make it possible for them to consent to anything may require acting before they have the capacity to consent (Tay-Sachs, Lesch-Nyhan). Perhaps, then, germline therapy is only permissible to replace mutant alleles that cause diseases that impair the patient's ability to consent to a cure. This proposal, too, is over-conservative. Imagine that gene replacement therapy becomes very exact (as envisaged above), that germline and somatic replacement therapies for myotonic dystrophy become possible, and that delaying the therapy until the offspring can understand and consent involves some irreversible weakening that both significantly diminishes physical capability and reduces expected lifespan. Here, too, there will be a case for intervening early, and, as in the extreme examples of Tay-Sachs and Lesch-Nyhan, for using germline replacement therapy.

Should we then draw the line by insisting on a distinction between curing a disease and enhancing a capacity? This is also problematic. Not only is the distinction notoriously hard to draw, but there seem to be instances in which something which is clearly an enhancement of a human capacity is morally permissible (Harris 1992). Suppose that genetic research teaches us how to enhance the operations of the human immune system, making people much less vulnerable to infectious disease and eliminating some susceptibilities to autoimmune disease. (Note that the judgment that the risks of gene replacement are low would have to be based on experiments with animal models, not on the restoration of normal human functioning since, ex hypothesi, no previous human beings have had the beneficial combination of alleles. Unless detailed understanding of nonhuman animals were available, the envisaged program of 
enhancement would be debarred by the considerations on risk developed earlier in this section.) Here again the benefits in terms of resistance to disease might be maximized if the intervention were made as early in ontogeny as possible, so that germline replacement therapy would provide the best opportunity for one's offspring.

Allusions to Frankenstein provide one way of articulating fears about gene replacement therapy; another centers around the charge that scientists will come to "Play God". Now it is quite correct that interventions in the germline would change the "natural" course of events. Life would no longer be "taken as it comes", and this might be thought somehow contrary to a proper appreciation of the sacredness of life. (This notion will be further discussed in section 8). Yet it is worth recalling that much scientific work is already devoted to interfering with the "natural" course of events. Great efforts are made to eliminate infectious agents and to immunize infants and small children against diseases. The envisaged strategy of enhancing the immune system should be regarded as continuous with such endeavors. We do not standardly view ourselves as morally compelled to accept nature "as it comes", and, while it is appropriate to be cautious in exploring the consequences of our interventions, our attempts to stamp out smallpox or to defeat the AIDS virus are not reasonably seen as insults to the sacredness of life. If gene replacement therapy appears different, that must surely be because of background worries about the identity of the people affected. Were the changes made in the genome sufficiently large in their effects to produce a different person, then, as noted above, the moral situation would be more complicated. If we suppose, however, that the enhancement of the immune system leaves the identity of the individual unaffected, the language of "playing God" should be seen as a dramatic way of describing a kind of action that attempts, quite responsibly, to improve human well-being, and to which many of our attempts to help people are already committed. 
So far, the idea that gene replacement, both somatic and germline, might be morally permissible in treating disease but not in enhancing capacities has been criticized on the grounds that it is too narrow. It is also too broad. For some conditions there is no premium on early intervention and the decision can, quite properly, be left to the offspring to make for themselves. Unless there is no doubt about how the people in question would choose to make that decision (as, for example, there might not be in the case of Huntington's disease), somatic replacement therapy is morally preferable to germline gene therapy in such cases.

The second of the questions distinguished above asked whether there should be limits on the kinds of therapies developed by researchers and advertised by doctors. One answer, consonant with the notion of individual reproductive freedom, would propose that gene replacement techniques should simply evolve subject to marketplace pressures: people would get the forms of therapy they want. Several writers (Powledge 1990, Paul 1992) have been concerned about the effects of any such market, noting, correctly, that while the relatively urgent needs of some might not be met (sufferers from rare genetic diseases), others might be sufficiently numerous to be able to satisfy frivolous desires. Even supposing that gene replacement techniques become virtually risk-free, there are grounds for opposing the development of processes that would straighten curly hair or generate faster-growing fingernails. Such processes would be a waste of health resources that might better be spent elsewhere and, if they were to succeed in attracting custom, their success would flow from the influence of arbitrary social attitudes that make it hard for people to accept themselves as they are. If there are genuine problems to be solved in this arena, they are surely social and not genetic, and they should be addressed by attending to the social causes of personal dissatisfaction. But it is also important to recognize that the difference between the frivolous and the important does not always coincide with the distinction between enhanced capacities and cured diseases. Some ailments -- like an allergy to hazelnuts - 
- are so minor that they can easily be endured without in any way affecting the quality of a person's life. Some enhanced capacities -- such as the envisaged improved immune system or the ability to resist memory loss with advancing age -- might greatly improve the lives that people lead.

Nonetheless, there are surely many kinds of capacities that we might envisage the scientists of the future trying to enhance, about which we have genuinely mixed feelings. Consider the possibility of increasing cognitive abilities. Some authors (Harris) have argued that we should think of gene replacement that would enhance the intelligence of individuals on the analogy of improved educational programs. Part of the resistance to this analogy comes from the thought that "intelligence" is not a welldefined notion, another part from the recognition that any foreseeable practice of intervention would be surrounded by enormous risks, yet another part from skepticism about identifying "genes for intelligence". Making some very large assumptions, let us suppose that it does become possible to identify a cognitive trait, intelligence, that uncontroversially improves people's abilities to deal with their environments and that enhancing this trait does not bring any unwanted side effects; assume also that a genetic basis for the trait is identified and allows for gene replacement therapy to enhance the trait; finally, suppose that gene replacement can be carried out exactly. Under these circumstances, are there residual worries about the envisaged gene replacement?

One source of concern lies in the thought that these opportunities will not be open to all. It would surely be wrong to enhance the abilities of the few before everyone in the population has the chance to benefit from programs designed to root out debilitating disease. Hence any enhancement of the type envisaged must be part of an approach that both attends first to the urgent health needs of the whole population and that ensures equal access to the enhancement of cognitive capacities. A second, related, worry is that efforts to improve people's intelligence are intrinsically antiegalitarian, that the desired goal would be to produce offspring who were more 
intelligent than the progeny of others. When subject to the constraint of equal access, some forms of enhancement are intrinsically self-defeating, since the aim is to secure some type of competitive edge: height and athletic ability are examples of traits for which this is plausible, and intelligence may belong in the same category. Finally, a third concern is that a widespread practice of enhancing cognitive abilities might generate a situation in which the lives of some people were less happy and productive. Lurking in the background is the thought that (in the terms of Huxley's Brave New World) a well-balanced society will have to contain both alphas and epsilons. Most people are repelled by the thought either of a population in which some people are frustrated because they cannot lead lives that are consonant with their abilities or of a planning policy that designs some people with enhanced abilities and others with diminished cognitive capacities.

Reflecting on these points, it appears clear that, when enhancing a capacity would have social implications were it to be implemented across the board, gene replacement therapy is morally impermissible unless there are conclusive reasons for believing that universal application of that therapy would bring about a society in which the lives of all were improved. Precisely because enhancing some of our psychological capacities would have such broad ramifications, we cannot confidently predict that the changes produced would be beneficial, or even that, with high probability, we would avoid social disaster. Some (but not all) imaginable therapies for enhancing traits confront us with risks of a different kind from those considered earlier -- where we previously thought about risks to an individual that could be measured by performing various kinds of biological tests, we are now forced to estimate risks of social disaster in unprecedented situations, and there are no readily available tools for making the requisite calculations.

The answers to the third and fourth questions distinguished above are already implicit in the foregoing discussion. There are many kinds of genetic replacement that 
might permissibly be considered with respect to a fertilized egg, but there is no moral justification for pre-empting future decisions that the person who will develop out of that egg would make, unless genetic intervention is needed to make possible future decision-making (as with syndromes that affect mental capacities), to prevent immediate effects that all people view as harmful to themselves, or to prevent a future condition that it is certain that the person will want to remedy and which can be treated more easily and more effectively by acting now. Similar conditions apply to the decisions that parents make on behalf of their children. Sometimes it is morally correct to intervene immediately. In other instances, when the consequences in the short run are negligible -- or when it is possible to make them negligible without altering the genotype - then the possibility of gene replacement should be left as a choice for the adult whom the child will become.

To summarize a complicated discussion, neither of the slogans "Treatment good, enhancement bad", or "Somatic good, germline bad" captures the proper moral constraints on gene replacement therapy. Instead, the foregoing reasoning suggests the following approach to particular instances in which gene replacement is envisaged.

(1) In cases of somatic therapy and germline therapy alike, it is important to calculate the risks of side effects.

(2) It is sometimes, although not always, possible to defer the decision so that that decision can be made by the person (or people) who would be affected by action taken in advance. If that can be done, then we respect their autonomy by doing so. If, however, it is certain that they would want the change that is to be brought about by advance action, and if that change is necessary to prevent conditions that would be damaging to them, it is justifiable to act in advance. Given that gene replacement therapy becomes sufficiently exact, germline gene replacement therapy would thus be justifiable in some situations. 
(3) Some forms of enhancement can clearly be predicted to increase the quality of life of future individuals. Germline gene replacement therapy to enhance such traits would be morally permissible, provided that the considerations of risk did not militate against them and provided that it was necessary to intervene before the people could make the decision for themselves. Many other forms of enhancement therapy have social consequences, which cannot be predicted, and these would be morally irresponsible.

(4) Potential gene replacement therapies should always be considered relative to (a) the impact of the replacement on the quality of the lives affected, (b) the equal availability of the therapy to all, (c) the importance of meeting the most urgent needs of all before attending to the less urgent needs of some members of society.

(1)-(4) provide guidelines -- though not determinate rules - for evaluating proposals to replace genes. Section 8 will contain some further discussion of the issues that they raise. 


\section{Forensic Uses of DNA Technology}

\subsection{Identifying People by their DNA}

Previous sections have explored the most obvious applications of the information that will be generated by the HGP, applications in medical contexts. DNA technology is already playing a role in other circumstances, most notably in the courts, and the HGP may fine-tune existing approaches to identifying people on the basis of their DNA. Except for monozygotic twins, each of us has a unique DNA sequence. Setting twins aside, it is thus possible to use information about DNA in a number of identification projects: to reunite families, to identify corpses, to check putative relationships, and, most commonly, to investigate and prosecute crimes. This section will concentrate solely on this last use.

DNA analysis can be used in forensic contexts in two different ways. One, restricted to the provision of evidence against an already identified suspect, involves the comparison of bodily materials (blood, semen, hair) left at the scene of a crime, with a sample (blood, a cheek swab) obtained from the suspect. The second, which can be employed as a means of discovery as well as evidence of guilt, consists in comparing the crime sample with a DNA database. With respect to each of these general methods, there are numerous ways in which the DNA analysis can be conducted. At present, the main strategy has been to look at regions of DNA that are highly variable (RFLPs or VNTRs, variable number tandem repeats, repetitive stretches of DNA based on a short unit such that the number of the repeating units varies from person to person). Often the DNA is amplified by PCR to obtain a collection of fragments that are then separated on a gel; sometimes, when the sample of DNA is sufficiently large, it is simply digested with restriction enzymes, yielding fragments for gelelectrophoretic separation; in the latter case, the crucial pieces of DNA are picked out using radioactive probes.) These molecular techniques are subject to specific problems of contamination, ambiguity, and misreading. Besides practical difficulties, there is 
the general theoretical problem of ensuring that the portion of DNA analyzed gives distinctive information about the person from whom it came.

If we knew the complete sequence of the DNA found at the scene of the crime and the complete sequence from the suspect, we would be clear about whether or not they matched. (There are some complications here, because mitotic divisions produce variations in the sequence present in somatic cells. However, two people are expected to differ by about 3 million base pairs, while the differences in the sequences of somatic cells are much less than this). Complete sequencing is presently impracticable, and, even given the most optimistic estimates of the development of sequencing technology, it is overwhelmingly likely to remain impracticable. (Besides the difficulties canvassed in sections 2 and 3 , there is the problem that the crime scene sample is often too small to make repeated analysis possible.) Hence, current forensic uses of DNA proceed by sampling.

Some regions of the genome show virtually no variation from person to person: coding regions are typically conserved, because modifications of the DNA sequence, apart from substitutions that do not affect the amino acid coded, can result in nonfunctional proteins. Comparing DNA at these regions is almost useless for purposes of identification. Conversely, the best parts of the genome to sample are those in which there is most variation. These will be regions least under the control of natural selection, regions of least functional significance. Some loci at which there is a high degree of polymorphism are already known, and it is probable that even partial sequencing of the human genome will disclose places at which the DNA sequence is highly variable. Collecting information about someone's DNA sequence in a region of high variability will typically not reveal anything at all significant about the person's phenotypic traits, because of the point made earlier: the functional stretches of DNA are highly conserved, the highly variable regions consist of nonfunctional DNA. 
Sampling DNA from a highly variable region thus serves both as an excellent diagnostic tool, and as an ideal "meaningless marker".

DNA typing presently proceeds by examining the restriction fragments or number of repeats in highly variable regions. Looking at a single region will not. usually suffice because, although there may be many alleles at a RFLP locus, there are not enough to make really fine distinctions. By considering several loci, the chance of a fortuitous match is reduced: two different people might have the same allele at a highly variable locus, but there is only a tiny probability that they will share the same alleles at $t w o$ highly variable loci, only a truly minute probability that the alleles will be the same at three highly variable loci, and so on. Even though we may be considering a very large population, focusing on multiple loci might set the probability so low that there is very little chance that anyone else in the population, besides the suspect, has the same combination of alleles as those found at the scene of the crime.

Perhaps future developments in sequencing technology will permit a different possibility. Instead of employing the relatively coarse divisions based on RFLPs and VNTRs, we might hope to discover fine-grained variations in the sequences of meaningless repeats with very long periods. Perhaps partial sequencing of the human genome will uncover regions in which virtually any base is likely to be modified. Sequencing a three-hundred base stretch from a region of this sort would pick one of $4^{300}$ possibilities (roughly $10^{180}$ ), any of which might be present in a person's genome. If it were possible reliably to pick out the pertinent stretch and to amplify it by PCR, we might be able to discover a diagnostic sequence for each individual by running a single gel, a diagnostic sequence that would, again, have the welcome property of being meaningless. For the moment this idea is only a dream, and we have to use multiple loci. 


\subsection{Failures of Independence}

Postponing until later questions that arise in connection with the possibility of technical error, the first problem to be addressed will be the theoretical problem of making appropriate inferences from the existence of a match. Mismatch involves no ambiguity: here the suspect can definitely be excluded. However, a match between the suspect's DNA and that left at the scene of the crime does not show that the sample at the scene came from the suspect, but only that it might have done so. Perhaps there is someone else in the population, whose DNA matches the DNA of the suspect at the loci tested. If we knew everyone's sequences at those loci, then we could determine whether or not this possibility is genuine. Without complete DNA typing, it is necessary to estimate the probability that the same alleles might occur in another member of the population.

The intuitive motivation for the method of multiple loci introduced the notion that if there is a small probability of sharing the same alleles at one locus, then the probability of sharing at two loci is tiny, and so forth. The natural way to develop this idea is to assume that the probability that sharing occurs at three loci is the product of the probabilities of sharing at each of the three loci, taken separately. In other words, if there is a chance of 0.01 that two people will share the same allele at the first locus, and a chance of 0.005 that they will share the same alleles at the second locus, and a chance of 0.002 that they will share the same alleles at the third locus, then the chance that they will share the same alleles at all three loci is

$$
0.01 * 0.005 * 0.002=0.0000001
$$

which is one in ten million. However, multiplying probabilities in this way depends on the assumption that the probabilities are independent. This need not be so, for it may be overwhelmingly likely that those who carry one set of alleles carry other sets. If independence fails, and if we have no further information about the probabilities that bearers of one set of alleles carry other sets, then the most we can say is that the 
probability is less than or equal to the lowest of the probabilities of sharing at the individual loci. In the worst case, all those who share the same alleles at the locus at which sharing is least likely (the third locus in the example) might also share the same alleles at the other two loci, so that the overall probability of sharing would be the smallest of the probability values ( 0.002 in the example).

This point can be made vivid with an analogy. Suppose that an eye witness to a crime testifies that the criminal has red hair, green eyes and freckles. The suspect has all three traits, and the prosecutor claims that only one person in one hundred has red hair, only two in one hundred have green eyes and only one in twenty has freckles. Multiplying the probabilities, the prosecutor claims that there is only a one in one hundred thousand chance that someone in the general population had all of these characteristics. But this calculation is clearly wrong, for we know that the probability of having green eyes if you have red hair is significantly larger than the general probability of having green eyes, and that the probability of having freckles if you have red hair and green eyes is much greater than the probability of having freckles period. Because the characteristics are not independent, simply multiplying the probabilities together gives the wrong estimate. People do not mate at random, and, in consequence, there are correlations both at the phenotypic level (red hair with green eyes and freckles) and at the genetic level. This is reflected in the fact that ethnic groups often have different constellations of physical characteristics and different susceptibilities to various types of disease. How, then, should one compute the probability that other members of the population will share the characteristics -genotypic or phenotypic -- found in the suspect?

For simplicity's sake; the discussion will consider a case in which three properties are shared between the suspect and the sample from the crime scene. It is easy to generalize to a larger number of shared features. The task is to find the 
probability that another member of the population would also have the shared characteristics. The general formula for the probability of a conjunction is

$$
\operatorname{Pr}(A \& B \& C)=\operatorname{Pr}(A) * \operatorname{Pr}(B / A) * \operatorname{Pr}(C / A \& B)
$$

where $\operatorname{Pr}(\mathbf{A})$ stands for the general probability of having the trait $\mathbf{A}, \operatorname{Pr}(\mathbf{B} / \mathbf{A})$ stands for the probability of having $\mathbf{B}$ given that you have $\mathbf{A}$ (the conditional probability of $\mathbf{B}$ given $\mathbf{A})$, and $\operatorname{Pr}(\mathbf{C} / \mathbf{A} \& B)$ stands for the probability of having $\mathbf{C}$ given that you have both $\mathbf{A}$ and $\mathbf{B}$. In the example of the last paragraph, the probability that someone has red hair, green eyes and freckles is the product of three probabilities: the probability of having red hair, the probability of having green eyes if you have red hair, and the probability of having freckles if you have both red hair and green eyes.

In the forensic context, it would be possible to calculate the probability that a member of the population will have a combination of alleles at three loci if we knew the conditional probabilities that particular alleles would be present at one locus given that particular other alleles were present at other loci. Typically, however, what are known are the general probabilities, reflecting the frequency with which particular alleles occur in a population. Calculating the probability that a particular combination of alleles occurs at a locus requires making assumptions about the patterns of mating within the population. (A fundamental theorem of population genetics enables one to compute the probability of a combination of alleles at a locus from the frequencies of the alleles in the population, given the assumption that members of the population mate at random. Of course, people do not mate at random, and, in applying population genetics to the problem at hand, it is necessary to estimate the extent to which there is divergence from random mating.) The most direct strategy, which would avoid controversial assumptions about patterns of mating, would be to find directly the pertinent conditional probabilities, and apply the theorem for the probabilities of conjunctions. However, to assemble all those conditional probabilities would be a truly Herculean task. Precisely the loci that need to be investigated are those that have 
a lot of variation (a high degree of polymorphism), so that the number of conditional probabilities needed per population is the product of several large numbers.

To argue that a match between the characteristics of the crime scene sample and the suspect's DNA is significant, prosecutors must show that the probability that other members of the population would also have DNA matching the crime scene DNA is very small. The method of multiple loci would work very smoothly if we had reason to think that the probabilities are independent. But it is extremely hard to establish independence. If the pertinent conditional probabilities were known, then the method of multiple loci could be made to work by employing the theorem for the probability of conjunctions. To gather all the necessary conditional probabilities would be an enormous task. Hence, prosecutors have to consult experts in population genetics to defend the claim that the idealizing assumptions of population genetics are approximately satisfied in the case at hand. In many instances, the estimates of the degree to which the approximation holds are controversial -- unless enormous statistical work is done -- so that the defense may rightly protest that there is a reasonable doubt that the match between the two samples of DNA shows what the prosecution claims.

The calculations so far reviewed attempts to determine the crucial probability on the basis of more elementary probabilities. The most direct way to obtain the needed information would surely be to sample a very large portion of the population and so discover the frequency with which a particular multilocus genotype occurs in it. The hope would be to show that nobody in the sample carries the pertinent set of alleles, so that we could conclude that the probability that another, uninvestigated, member of the population bears the same genotype is less than the reciprocal of the sample size (thus, if the sample is of size $N$, the probability is less than $1 / N$; if the sample is sufficiently large in comparison with the unsampled portion of the population, the probability that the unsampled part of the population contains a match will be low; more exactly, that probability will be less than $1-[(N-1) / N]^{M}$, where $M$ is the size of the unsampled part 
of the population.). Prosecutors have often flourished dramatic probabilities in the courtroom, suggesting that the chance that anyone other than the suspect would match the sample from the crime is less than one in some astonishingly high number (millions, billions, even trillions). These minute probabilities are calculated by multiplying small numbers and assuming independence - thus they can be challenged in the ways described above. The more direct method would not yield as much drama, for it is impractical to sample millions, let alone billions or trillions. Yet, as will become clear in $\$ 7.4$, nothing significant may be lost by using more mundane figures. For further discussion of the issues raised here, see (Lander 1992, McKusick 1992, Lewontin and Hartl 1991).

\subsection{Identifying the Population}

In formulating the problem so far it has been assumed that there is no ambiguity in the notion of "the population". However, it is well known that many alleles vary significantly in frequency from population to population. The claim that the prosecutor has shown that "the probability that someone else in the population would have matched the sample left at the scene of the crime is very low" is thus in need of clarification. Which population is pertinent here?

The relevant group of people are surely those who could have committed the crime. Forensic scientists should thus carry out their sampling on a population that accurately represents the people in the vicinity at the time of the crime. (Moreover, it is crucial that this population should be sufficiently small to allow fairly complete sampling. If the population is large in comparison with the sample, then, although the probability that any particular uninvestigated person shares the multilocus genotype may be small, there may be enough uninvestigated people to confer a significant probability on the claim that some uninvestigated person shares the genotype. In one of the most famous uses of DNA analysis, the detection and conviction of Colin 
Pitchfork, in Leicestershire, England, the police attempted to obtain samples from the entire local male population: ironically, Pitchfork was caught when it was discovered that he had persuaded someone else to contribute a sample for him.) Sometimes it may be relatively straightforward to see how to circumscribe this population. But, in other instances, it is not. Richard Lewontin poses the problem by reference to a case of "lethal assault" that took place in Northern Vermont.

The victim, like the accused, was half Abenaki, half French-Canadian and was assaulted where she lived, in a trailer park, about one third of whose residents are of Abenaki ancestry. It is a fair presumption that a large fraction of the victim's circle of acquaintance came from the Indian population. No information exists on the frequency of DNA patterns among Abenaki and Iroquois, and on this basis the judge excluded the DNA evidence. But the state could easily argue that a trailer park is open to access from any passerby and that the general population of Vermont is the appropriate basis of comparison. Rather than objective science we are left with intuitive arguments about the patterns of people's everyday lives. (Lewontin 1992 78).

This challenge is important. If the DNA profile found at the scene of the crime matches the DNA profile of a suspect, we cannot draw conclusions about the probability that that profile will be present among other people without thinking carefully about the potential population of suspects. In the case discussed, we ought to reject the claim that the chance is less than $1 / 1000$ on the basis of a sample of the general Vermont population containing only five Abenaki members. More generally, if small populations, with distinctive associations of genes, are underrepresented in the sample, then it is fallacious to claim that there is a high probability that the sample came from a unique individual.

To solve this problem, it is necessary for the prosecution to demonstrate that it has seriously considered the variety of ways in which the criminal had access to the 
victim. Continuing with Lewontin's example, we can recognize that the crime might have been committed by a passing motorist, by a member of the local population, by a member of one of the nearby ethnic populations (such as the French-Canadian population across the border). Some of these populations (such as the local inhabitants) are sufficiently small to allow for near-exhaustive sampling. Others would be sampled to determine upper limits on the probability of a match.

Even if prosecutors work hard to explore a variety of hypotheses about the population from which the criminal came, showing that, in these populations, the probability that another person's DNA profile would match that of the crime scene DNA is less than $1 / 1000$, it is still important to set their precise figures in context. The final estimate is only as good as the reasoning that underlies the concentration on particular groups of potential suspects. If there is reason to wonder if some hypothesis about likely suspects has been neglected, thus underrepresenting some people among whom the multilocus genotype might be more frequent, then the case will not have been made (Lewontin 1992, Nelkin and Tancredi 1989). Perhaps DNA evidence has seemed to replace qualitative considerations with numerical precision, but it is important to remember that the exact figures only gain their force against the background of traditional types of legal argument which show why it is right to obtain statistics from particular populations.

\subsection{Laboratory Error}

The extraordinary figures that have sometimes been presented as estimates of probability may not only be miscalculated (for reasons given in \$7.2) but also meaningless. For in each case of forensic testing, it is necessary to take into account the probability of laboratory error. Not all kinds of laboratory errors would result in the appearance of a match: some mistakes issue in null results that wear their failure on 
their faces. If, for example, a technician fails to adjust the temperature for a PCR or lets a gel run too long, it will be very obvious that something has gone wrong.

The significant errors are those in which a sample from a suspect that does not match the crime scene DNA is seen as matching. The simplest way for this to occur is through mislabelling or contamination: the two profiles are thought to match because, in effect, the experiments have taken the same DNA and investigated it twice. Forensic scientists can guard against such errors by testing the crime scene DNA and the suspect DNA in separate laboratories, and the prosecution's case is strengthened if this is done. More generally, given the early troubles experienced by Lifecodes and other professional testing laboratories, it is important to introduce standards of quality control and to demand that forensic testing be subject to periodic processes of evaluation (Lander 1992, McKusick 1992). Calibrating success in determining matches and avoiding false positives is not difficult. Laboratories can be sent coded samples and asked to determine which ones match, where this information is already known to the evaluators: having drawn blood from a number of people, they know that numbers 7 and 13 came from the same person, as did 5 and 11 , and that none of the others should match.

There is no point in using sampling to generate probabilities of less than $1 / N$ if the rate at which false positives occur in a laboratory is greater than $1 / N$. Experience with juries demonstrates that a probability of $1 / 1000$ is quite low enough to satisfy virtually all interpretations of "beyond a reasonable doubt". To show that the probability that a given multilocus genotype occurs in a particular population is less than $1 / 1000$ would require demonstrating that the genotype is absent in a sample of one thousand members of the population, provided that the population size is not much greater than this. This is sometimes a manageable task. Similarly, it is not overly optimistic to suppose that quality control of testing laboratories can produce rates of false positives that are below $1 / 1000$. Thus a practical approach to the problem of 
showing "beyond a reasonable doubt" that the sample at the crime scene came from the suspect is to combine three safeguards:

(A) Identify a number of candidate populations, for which statistics are to be given, arguing, on the basis of traditional kinds of legal evidence, that the criminal came from one of these populations.

(B) Show that, in each of these populations, the probability that another member of the population would have had the same DNA profile as that found at the scene of the crime is very small. This should be done by sampling the frequency of the multilocus genotype within the population.

(C) Introduce and implement quality controls for the laboratories involved in the testing, so that there is a basis for claiming that the probability of a false positive (an apparent match where there is no true match) is smaller than the probability generated in (B).

Even under these circumstances, it might be possible for the defense to argue that the procedure in (A) has overlooked an important class of potential suspects or that one or more of the populations considered is large enough so that, even with a low probability value in (B), there would be a significant number of people in the population whose DNA profile might match that of the crime scene sample. DNA evidence can be used as part of a cogent case, but it is important for judges, juries and lawyers to recognize that the probative force of that evidence depends on qualitative.considerations that have not been superseded by the numbers.

\subsection{Population-Wide Typing}

It is possible that the HGP will make it possible to refine the method of multiple loci by identifying regions of the human genome at which the variation is so extensive that we can be confident that no two individuals share the same DNA sequence. To attain that kind of confidence would, of course, require large-scale sampling of the US 
population. However, it is possible, in principle, to free forensic uses of DNA from the dependence on statistics and legitimate concerns about whether or not the statistics represent the appropriate population. If there were a DNA profile for everyone born in the USA -- and for every person entering the USA - then there would be no need to estimate the chance that someone other than the suspect had a genotype matching the DNA in the crime scene sample. Abstracting from complications involving monozygotic twins, it would be possible for police officers to search a database to find the unique individual whose DNA matched that found at the crime scene. Evidence similar to that employed in discovery could then be used in the courtroom, with the only danger of error stemming from the possibility of mistakes in the laboratory.

Many people find the idea of a national DNA database appalling. A standard reaction is to condemn the notion as an unwarrantable invasion of privacy. However, before it is dismissed, it is worth considering carefully what such a database might involve, how it would be generated, and how it might be used.

It would be possible to type everyone in the USA in the following manner. Using clues provided by the partial sequencing of the human genome, we would look for loci of the highest variability, subject to the constraint that DNA at these loci could be easily and reliably amplified by PCR. It will surely be the case that we can find a number of loci at which the most frequent genotype (= pair of alleles) occurs at a frequency of $1 / 10$. If ten such loci are chosen, then, assuming independence, the number of ten-locus genotypes will be $10^{10}$, more than enough to provide a unique genotype for every member of the population. Because of breakdowns in independence, the actual population may contain only a small fraction of these combinations, but if it is discovered, in the course of typing subpopulations, that the ten loci are insufficient to ensure a unique genetic profile for everyone (except monozygotic twins, of course), then we can add more loci, or seek loci with even more variability. To fix ideas, suppose that the loci chosen involve variable numbers of 
short repeats, and that ten loci prove sufficient. Then each member of the population can be represented by a short sequence of numbers: thus the code for a person might be 37221613347915872611

where each term of the sequence represents the number of occurrences of the repeat at a particular locus (the person has 37 copies of the repeat at the first locus, and so forth). It is important that the loci should be tested for stability, that is, when we take samples from the same person, we always find the same number of occurrences at each locus.

Each person can be typed by entering the pertinent sequence of numbers in a database, with the name attached. The samples from which the numbers were obtained can be -- and should be -- destroyed, so that no further information can be obtained from them. Hence the database gives no clues to exciting properties that people may have: for the fact that someone has 37 repeats where another has 51 has no significance whatsoever. (There may be some slight complications here, in that numbers of repeats at a locus may give clues about ethnic background. This suggests a further constraint on the choice of loci: we should try to find loci at which intragroup variation approaches intergroup variation. It should be possible to satisfy this constraint.)

How could a database like this be constructed? It could easily become standard practice to type all newborm infants, and to require either a blood sample or cheek swab (or the prior submission of typing information) from any one who seeks entrance to the country. Adults who had not been typed as children could be required to attend medical centers at which samples would be taken at no charge, and the demand could be enforced by making it necessary to show evidence of having been typed before obtaining a driving licence, receiving unemployment, entering a hospital, and so forth. Considerable work would obviously be involved in ensuring that those people most 
loosely connected with the social system are typed, but the problems are not insuperable.

Suppose now that a crime has been committed and a DNA-bearing sample is left at the crime scene. The DNA would be amplified at the crucial loci, and the number of repeats at each locus would be determined. The ten-number sequence would be entered in the database for a possible match. If there were a match to the profile of a single individual in the population, then the police would use standard methods to find the person, and would now be permitted, by law, to demand a further sample. That sample would be checked at a different laboratory, one that had examined neither the crime scene sample nor the sample used in the original typing. If the match with the crime scene sample were sustained, the new sample would be retained for use as evidence at the time of trial.

The design of the database and the procedures surrounding its use together circumvent some of the problems encountered with existing courtroom uses of DNA evidence. The first point to note is that a database can serve both in exonerating or convicting people and in identifying suspects. Second, the considerations about probability are banished: except for monozygotic twins, the typing system is set up so that each person legally within the borders of the USA has, on record, a unique DNA profile. There should no longer be concern about computing probabilities by assuming independence, or about large scale sampling of populations to discover the frequency of multilocus genotypes. Third, the envisaged procedure eliminates ambiguity about the population within which matches are sought. To the extent that the efforts to type everybody within the USA's borders are successful, everyone is on record. The only residual doubt about a match is that someone has avoided being typed, and this person, by chance, has the same DNA profile as the suspect. If two hundred million people have been typed, and the only shared DNA profiles occur in pairs of monozygotic twins, then this possibility is hardly a serious one. Fourth, destruction of the samples 
used in typing means that those samples cannot later be employed to garner further information about citizens (or legal visitors). The only information on record is thoroughly meaningless. Fifth, separating the typing laboratories from the crime scene laboratories, and both from the laboratories that analyze samples taken from suspects, is an important way of safeguarding against false positives. As already noted, the most frequent method of obtaining false matches is through mislabelling or contamination. Sixth, mistakes in typing, which are almost inevitable when a large population is typed, can be detected by requiring that a sample from the suspect be reanalyzed, by an independent laboratory. A match between the crime scene sample and the database only confers the right to demand a further sample. Perhaps there will be further grounds to arrest the suspect, but, if such grounds are lacking, police must wait for the new sample to be checked.

Although the proposal method of constructing and using a nationwide DNA database responds to some of the worries that many people have, serious concerns remain. The National Research Council's report on DNA Technology in Forensic Science considered, but did not endorse, the idea of a national database (McKusick 1992 121-122). Two main reasons were given: the first centers on concerns about privacy, the second on issues about cost. These worries will be taken up in order. As noted in $\$ 5.1$, privacy has both intrinsic and instrumental value. How would a national DNA database interfere with either of these values? There are conceivable, but far-fetched, scenarios in which those with access to a database use knowledge of DNA profile to track a person's movements: these are far-fetched because the work involved in finding and analyzing DNA samples seems grossly disproportionate to the value of the information gained. Far more pertinent is the thought that corrupt law officials will substitute a blood sample from an innocent suspect for the crime scene sample, thus framing those they wish to convict. However, there is nothing genuinely novel here: corrupt police officers already have 
means of incriminating the innocent. If a national DNA database is viewed as forfeiting an important instrumental value of privacy, that must be because of fears that dishonest officials would now have an instrument of far greater power available to them. Of course, any truly effective forensic evidence would be vulnerable to abuse in the same way. As things now stand, it is possible to claim that samples from the suspect were found at the scene of the crime, and, to the extent that the match between these samples and new samples taken from the suspect provides powerful evidence, framing is possible. Hence, the concern about misuses of the database stems from the thought that it will provide extremely powerful evidence of guilt -- precisely its evidential strength is the source of the fear. It is now possible to see that the objection rests on an uncomfortably schizophrenic attitude, torn between wanting police to have effective tools for finding and convicting the guilty and fearing that those tools might be abused. The remedy is obvious in principle, although not necessarily easy to construct in practice. If a national DNA database interferes with the instrumental value of privacy, it does so because we lack sufficient safeguards against misconduct by the officers of the law, and, if that is indeed our predicament, our first task should be to erect such safeguards. If we do not do so, we are already in trouble.

What of the intrinsic value of privacy? Some information about ourselves, our emotional reactions, our needs and wants, needs careful protection: we do not want others, except for those with whom we are most intimate, to know these things, even if they do nothing with the information to harm us. Yet, whether or not some information that might become available through routine analyses falls into this category, broadcasting of the lengths of meaningless repeats is of utterly no significance. To a first approximation, the kind of information we want contained, simply for the sake of keeping it private, covers things that are central to ourselves and our sense of ourselves. Only those who have extraordinarily distorted self-conceptions. 
will suppose that having 37 CG repeats rather than 11 or 51 in a particular region of nonfunctional DNA is central to their being the people they are.

There are deeper, more nebulous, concerns, based on the vague feeling that a national DNA database would resemble Orwell's "Big Brother", creating a world in which all citizens were treated as suspects. It is salutary to oppose this vision with a contrary perspective. $\$ \S 7.2-7.4$ showed that a potentially efficient method of convicting the guilty cannot reliably and justly be employed without the cooperation of the innocent: without comparisons between the matching samples and the DNA of members of broader populations, it is impossible to know whether or not a match might appear with others. Even as things stand, innocent people must contribute information about their genomes so that they can be distinguished from the guilty. There is no presumption here that all are guilty, but rather a tacit agreement that the innocent will work together to enable the singling out of the guilty.

This point can be developed further by considering an intermediate strategy, which has become popular in some states and some sectors of society. Instead of using statistical inferences in the way considered in $\$ \S 7.2-7.4$, and instead of constructing a complete national database, states and institutions (such as the armed forces) are generating partial databases. In many states, typing of convicted felons has begun. This approach to problems of detection can appear attractive because, first, it is cheaper to perform DNA typing on a subset of the population, and, second, proponents argue that most crimes are committed by recidivists, so that the extra work and expense of typing the entire population brings no significant returns. Moreover, any construction of a partial database avoids the charge that government has invaded the privacy of the innocent.

Partial databases can be expected to work in the following way. A sample from the crime scene is matched with a DNA profile in the database, the suspect is found, a new sample is taken, and this matches the crime scene sample. If this 
evidence alone is now used to convict the suspect, then there should be serious doubts about the possibility of another match in the unexamined part of the population. The claim that most crimes are committed by recidivists could easily become a selffulfilling prophecy. We identify criminals in terms of convictions; we generate convictions by comparing the DNA profile of a crime scene sample with the profiles in a database restricted to previous criminals; since these are the people who are investigated and who are convicted on the basis of DNA matches, it is hardly surprising that they would be the majority of those convicted; the view that most crimes are committed by recidivists would thus become an artefact of our method of catching and convicting those we call "criminals".

It is possible to escape this unjust circularity. One approach would be to use DNA matching to find suspects, but to use other evidence to convict. Alternatively, it would be possible, in each case, to buttress the evidence from the database with population-wide statistics of the kinds discussed in \$\$7.2-7.3. The first approach undercuts the probative value of DNA evidence -- although it might provide the basis for using such evidence in the future if the reliability of using DNA matches with a partial database were sustained by the regular convergence of DNA evidence and more traditional types of evidence. The second option leads back into the difficulties, and expense, of sampling appropriately chosen populations in an attempt to show that concentration on those already convicted does not produce a bias. The clearest and most reliable way to evaluate the claim that, when DNA profiles match, we have overwhelming evidence for maintaining that the crime scene sample came from the suspect, is to show that the kinds of DNA profiles constructed really do individuate members of the US population -- and, for that, a national DNA database would answer all doubts.

There is no doubt that universal DNA typing would be expensive. Assuming a cost of $\$ 400$ per person, it would cost $\$ 100$ billion to type a population of a quarter of 
a billion people. This would be a one-time expense, with smaller follow-up expenses dependent on the birth rate. The cost must be measured against other costs incurred in criminal investigation, including the use of other kinds of forensic evidence and the routines of searching for clues that might be replaced by the use of a database.

Moreover, in evaluating the possibility of a national database, a partial database, or continuation of existing uses of DNA evidence, it is important to take into account the need to sample the general population if either of the latter options is pursued. Which option makes most economic sense depends on the frequency with which crimes lend themselves to forensic uses of DNA, and the extent to which those uses can eliminate current routines with high costs. It is crucial, however, that economic considerations should not be allowed to override the demands of justice. Developing a procedure that is powerful while safeguarding the rights of the accused is expensive, but it would plainly be unjust to dismiss statistical niceties in the interests of cutting costs and, quite possibly, the cheaper methods will reduce the power of forensic DNA evidence because juries are loth to convict when the defense can raise doubts that the statistics assembled are possibly incomplete or biased.

\subsection{The Scope of Forensic Uses of DNA}

The discussion so far has taken it for granted that DNA analysis, if properly conducted, would provide a powerful technique for detecting and convicting criminals. Given the complexities uncovered above, it is worth scrutinizing this assumption. In his original brief for forensic uses of DNA technology, Alec Jeffreys suggested that it might provide a foolproof method of detecting and convicting perpetrators of violent crimes. The natural thought is that this would lower the crime rate, at least by confining violent offenders and perhaps through deterrence.

In most instances of single rape, DNA samples from the criminal can be collected and analyzed, since a vaginal swab from the victim will yield sperm carrying 
DNA from a single source. Multiple rapes are more complex because of the difficulties of analyzing DNA from several sources, but these problems can sometimes be overcome. With assaults and murders, police have to depend on blood spilled at the scene of the crime or on cells from the criminal that may be found on the victim (most notably, blood and skin under the victim's fingernails). Sometimes there may be no direct contact between the victim and the assailant, and on these occasions there will be no obvious way to detect any organic matter that the criminal -- as opposed to other people -- has left at the scene.

In all these examples there are complexities that affect the route from discoveries made at the scene to the conclusion that someone committed a certain crime. If a sample is discovered, and if DNA analysis is properly employed to reveal a match with a person, we can infer that that person was at the scene (assuming, of course, that the sample has not been left there by someone else). To produce conviction it is also important to show that a crime was committed and that the person was not only at the scene but also involved in the action. Single rapes provide the most straightforward examples, for here the rapist's semen is (typically) found in the victim's vagina, so that there is not simply an association between the criminal and the scene. However, as is abundantly clear from the course of many rape trials, there is an inferential gap between the recognition that a person engaged in sexual intercourse with the victim and the conclusion that the act constituted rape. If, in many cases of rape, rapists are acquitted because juries cannot rule out the possibility that the act was consensual, then the goal of convicting virtually all rapists will not be attained, even if DNA technology is applied in its most powerful form. (It is worth pointing out, however, that children who are victims of incest may be empowered by forensic uses of DNA, and may be able to show conclusively that they have been sexually abused.)

Cases of violent assault and murder involve problems of a different kind. Here there is no doubt that crimes have been committed; the difficulties surround the 
discovery of samples for DNA analysis and the establishment of a connection between presence at the scene and the criminal act. If it is clear that an organic sample matching the suspect is found where it is because of a retaliatory act on the part of the victim -- as when, for example, blood and skin are found under the victim's fingernails - then the connection can be made. But when the presence of the organic material can be explained in other ways, as when hairs from the head of the victim's spouse or companion are found on the victim's clothing, the existence of a match does not compel the conclusion that the suspect committed the crime.

Successful introduction of forensic uses of DNA may itself make it harder to establish the crucial connections. Relations between violent criminals and their victims are not likely to remain stable in the wake of improved forensic techniques. Just as the practice of taking fingerprints caused burglars to take steps to avoid leaving prints at the scene of the crime, so we can expect that rapists, murderers and assailants will change their ways to try to circumvent being linked to the crime through their DNA. The strategy will have to be different, for it is impossible to avoid leaving some organic matter behind. Nonetheless, criminals who plan their actions can try to avoid leaving readily detectable organic samples that would link them to the victim (rapists can use condoms, murderers can cover themselves to prevent the possibility of being clawed), and they can also take advantage of the fact that samples of others' DNA (unlike others' fingerprints) are portable. Premeditated crimes can be carried out with an eye to confounding police attempts to isolate the criminal's DNA: sufficient amounts of blood, hair, and/or semen can be distributed at the crime scene. If these scenarios seem unlikely, it should be noted that there are already anecdotes of New York prostitutes' starting a market in used condoms (Nelkin, personal communication). Some crimes, of course, are unpremeditated, and, on these occasions, forensic uses of DNA may work well to identify criminals from the traces that they leave. Furthermore, even though a criminal is trying to avoid leaving detectable samples or to 
confuse the evidence by distributing misleading samples, the victim can take steps to ensure that DNA from the criminal is left behind, and that it can be identified as the criminal's by the police. Victims who survive the crime can guide detectives to the appropriate places. Even dead victims can speak to avenge the crime, if clawing and biting the murderer forces samples to be left on teeth or nails. Of course, there is no guarantee that these tactics will succeed, and they may even escalate what is already a horrifying encounter, but potential victims do have some power to threaten. Whether or not that power is sufficient to deter an attacker will depend on circumstances.

Victims will have to make snap judgments about whether efforts at retaliation are likely to cause the criminal to flee or whether they will lead to their own death.

Jeffreys' forecast about the detection of virtually all violent crime through the use of DNA analysis is thus too optimistic. Successful uses of DNA technology would begin an arms race between criminal and victim, and it is quite unclear who would win. In those segments of society in which the threat of conviction and imprisonment exerts little, if any, deterrent effect, it is unlikely that criminals who premeditate their crimes can be prevented from taking steps to elude forensic DNA techniques. Those techniques may still serve more limited purposes, increasing rates of detection and conviction by amounts that are currently unpredictable.

\subsection{Options}

There are several options for the future role of DNA analysis in forensic contexts.

(1) Eliminate the use of DNA evidence entirely.

(2) Use DNA evidence in something like its present form, recognizing the problems associated with evaluating the probability that the match arose fortuitously. 
(3) Extend the use of DNA evidence by constructing a partial database with records of the profiles of convicted felons.

(4) Construct a nationwide database and apply it along the lines described in $\$ 7.5$.

Which option should be preferred? (1) seems too sweeping a suggestion unless it is demonstrated that we cannot proceed in an ad hoc way under the rubric of (2), introducing DNA evidence for just those crimes that permit us to respond to the requirements of justice in a cost-effective way. The advantage of (2) is that it does not commit us to a project involving large costs. Its danger is that considerations of expediency will override the need to show, beyond reasonable doubt, that other members of all the relevant populations do not have DNA profiles matching the crime scene DNA. Those who favor (1) surely do so because they believe that the rights of suspects, especially suspects from certain ethnic and racial groups and socioeconomic classes, will be slighted by compiling inadequate statistics. So long as there are scientists who believe that statistical shortcuts are being taken, and who are willing to serve as expert witnesses, the apparently "clean" DNA evidence will be inextricably bound up with judgments of the relative credibility of experts, judgments made by jurors who have no basis for judging the highly technical issues themselves.

Option (3) involves considerably more expense and promises greater powers of detection. However, to implement it in a just way would require attention to exactly those statistical analyses of broader populations that are needed as part of the case-bycase approach pursued in (2). Option (4) is the clearest way to honor legitimate concerns about justice, but it would be extremely costly. Initially, it might be thought that these costs would easily be justified by the great reductions in rates of violent crime that DNA analyses would make possible (and, perhaps, by reductions in rates of other forms of crime as well). However, the consequences of a sustained program of using DNA analysis in detection and conviction are far from obvious. At present, it 
would appear that the most promising options are either (4) or a version of (2) that is sensitive to the range of considerations adduced in $\$ \S 7.2-7.4$, but firm conclusions are unjustified until there has been a thorough attempt to think through the role of DNA evidence in the context of an overall policy for the prevention and detection of crime. The present discussion can only raise the pertinent issues that need to be considered carefully in that broader context. 


\section{An Overview of Some Further Issues}

\subsection{Introduction}

Besides the questions taken up in earlier sections, there are further issues that should be addressed both to complete discussion of the most immediate ethical and social implications of the HGP and to understand the longer-term impact of the increases in our knowledge of molecular biology which the project will bring. Most ELSI attention has so far been focused on making genetic testing as valuable as possible, preventing genetic discrimination, developing guidelines for therapeutic interventions and scrutinizing the uses of DNA evidence in the courtroom. However, it is possible that, within a few decades, these concerns will appear parochial and myopic compared to the more abstract and general issues raised by the HGP.

This section will only attempt to begin discussion of four such issues. It will raise, in a very preliminary way, questions about genetic determinism, about eugenics, about the project of planning populations and the role of abortion in so doing, and, finally, about how priorities in medical research might be set, both within the USA and within world-wide discussions. At the moment, these questions do not call for the development of laws and policies, although it will be important to begin discussing them now if the most deep-seated fears about the HGP are to be allayed.

\subsection{Genetic Determinism}

Molecular biological research is sometimes charged with over-emphasizing the role of genes, with supposing that phenotypic traits of organisms -- including human beings -- are genetically determined. Virtually all biologists publicly disavow genetic determinism, insisting, as have earlier sections, that both genes and environments are involved in the formation of phenotypes. Nonetheless, it is standard to talk of genes for phenotypic traits -- genes for PKU, genes for cancer, even, sometimes, genes for aggression and genes for homosexuality. This talk, especially as it is reported in the 
media, encourages many people to think that many of our traits, including our behavioral traits, are determined by our genes, and that, in consequence, there is little that can be done to alter them by changing the environments in which people grow up and live as adults. Because public policies often depend on the idea that human lives can be improved by modifying environments -- providing educational resources early, improving housing conditions, diminishing the blight and the violence that many young children experience daily -- it is important that the exact implications of the molecular biologist's appeals to genes for such-and-such should be recognized.

The way in which any phenotypic trait of a multicellular organism is caused (the causal pathway underlying the trait) is highly complex, and, except for a very small number of traits of a very few (non-human) organisms virtually all the details are unknown. Because of the presence of particular molecules within the fertilized egg (proteins deposited in the cytoplasm by the mother), some genes are switched on, making further proteins; as a result of this internal activity, the cell divides; more genes are transcribed and translated, the protein constitutions of cells change, cells interact with one another and with the environment external to the organism; eventually, out of billions of billions of local reactions, there emerges an organism with identifiable characteristics. Yet, despite all this complexity, there are some regularities which geneticists can identify. Sometimes, almost whatever the genetic background, and almost whatever the external environment in which the organism develops, an organism bearing a particular allele will have a particular phenotypic trait. In such instances, it is tempting to think that the contribution of the environment can be neglected, that the genetic background does not matter, and that the trait in question is determined by the allele.

Huntington's Disease is an obvious example. Those unfortunate people who have the HD allele (or, more exactly, an over-long CAG repeat) near the tip of either copy of chromosome 4, will apparently develop the disease no matter how they live. 
Sufferers have different genetic backgrounds; they grow up, and live as adults, in different ways. All this seems to make no difference. The sad termination of their lives is fixed by the presence of the allele.

Now, in fact, the characterization of Huntington's Disease given in the last paragraph, while initially plausible, is strictly false. First, it is not inevitable that anyone carrying the HD allele will develop the disease: some HD bearers die before they would have begun the process of degeneration. This, of course, is not comforting, and shows only that some environments which prematurely terminate a person's life do not allow the expression of the HD allele in the disease. Far more importantly, the search for ways to prevent and treat the disease reflects the idea that there are presently unknown environments - possibly involving modifications of the HD-bearer's body in ways unanticipated as yet - in which people carrying the HD allele would not suffer from the disease. Thus it would be more exact to say that, from our present perspective, the disease appears fixed by the allele, in the sense that we do not know how to produce any environment in which HD-bearers survive past the typical period of onset and remain free of the disease.

To sum up and generalize, a characteristic is genetically determined by an allele (or combination of alleles) when there is no combination of other alleles and no environment in which it would be possible both for the bearer of the allele (the combination) to survive beyond the stage at which the characteristic would appear and to avoid having the characteristic. (If you change the genetic background and/or the environment then the organism still has the trait unless the change causes it not to survive to the time of expressing the trait). A characteristic appears genetically determined by an allele (combination) when there is no known combination of other alleles and no known environment in which the bearer can be enabled to survive but still avoid the characteristic. (For some kinds of characteristics it may be important to replace the notion of survival with that of "thriving" or "exhibiting normal function", 
but these refinements will be ignored.) In searching for ways to treat disease, researchers who apply contemporary molecular biology (including the results from the HGP) are assuming (hoping) that conditions that currently appear genetically determined are not in fact genetically determined.

As already noted, much contemporary molecular genetics (as well as much classical genetics) talks of genes for various traits. Does this kind of discourse reflect any commitment to genetic determinism? The root idea of a gene for a phenotypic trait is that mutation at a locus produces differences in the trait. Geneticists investigating a particular species discover that organisms with the usual kinds of alleles at other loci and living in the environments in which they find members of the species display an abnormal phenotype if they have one or two unusual alleles at the locus in question. Because they cause an abnormal phenotype, against a standard genetic background and in a standard environment, the unusual alleles are said to be "for" the abnormal phenotype, and the alleles at the locus that produce the normal phenotype are said to be "for" the normal phenotype (Sterelny and Kitcher 1988).

Several consequences of this usage should be clearly understood. First, talk of genes for sophisticated forms of behavior does not conflict with the idea that those forms of behavior are brought about in complicated ways. There might, for example, be genes "for" dyslexia, in the sense that there is a locus at which mutant alleles occur, so that, when a person has a pair of these alleles, if that person has a normal genetic background and grows up in one of many standard ways, being introduced to printed materials in much the way children usually are, then that person will exhibit the reading and spelling problems characteristic of dyslexia. The normal alleles at this locus might come to be known as "reading alleles" or as "nondyslexic alleles". None of this violates the obvious point that developing a capacity to read is an extraordinarily complex process in which, no doubt, innumerable genetic and environmental factors are involved. 
Second, there can easily be genes "for" traits which are not genetically determined. Assuming that reading and spelling difficulties can be addressed by imaginative educational programs, it is possible that bearers of alleles for dyslexia can find themselves in environments that enable them to avoid the usual effects. The case of PKU furnishes another clear example: in the historically inherited ways of nurturing, the allele always yields a phenotypic condition (severe retardation). Since medicine has been able to prevent the retardation in those who bear the allele, it is possible to carry an allele "for retardation" and develop normally. Of course, an allele originally identified in a particular way, through some phenotypic effect that is deleterious to those who bear it, can be reclassified once ways of avoiding the phenotype are known. It is now strained to think of the PKU allele as "for mental retardation" (in double dose). We more naturally think of the allele as contributing to an inability to metabolize phenylalanine, and of the disturbance of development as separable and avoidable.

Third, it is possible that there should be a gene for a phenotypic trait even though most instances of that trait have nothing to do with the presence of that gene. People who have high levels of LDL cholesterol do so as the result of very different causal pathways. At one extreme are those who carry a combination of alleles that, in all the environments we naturally inhabit (that is, without special forms of intervention), cause them to have livers that are unable to break down LDL cholesterol. At the other extreme are people who carry all the normal alleles at the loci implicated in the causal pathways that underlie the production and breakdown of cholesterol, and whose elevated levels stem from dietary excesses alone. Between these poles there is likely to be a large range of cases, in which there are interactions between genetic and environmental factors. Although there may be a gene for elevated levels of LDL cholesterol - the allele borne by people at the first (genetic) extreme -- the 
overwhelming majority of instances may not be due to the presence of that gene, and, further, many of them may be purely environmental. To sum up:

- The fact that there is a gene "for" a trait is quite compatible with (a) the trait's being brought about as a complex interaction between genes and environment, (b) the trait's not being genetically determined, and (c) the trait's being caused in quite different ways in different organisms, with some of these instances being caused by the interaction between normal genes and environmental factors.

With these points in mind, it is possible to clarify the notions of genetic disease. Two main notions have figured in the literature, one illustrated by diseases like PKU and Huntington's disease, the other by the various cancers. The first conception of genetic disease covers those conditions that are always brought about, in the standard environments that we have inherited, by the presence of a particular genotype. In accordance with points made above, it is quite possible that there should be ways of modifying the environment so that the disease phenotype is not produced, even when the combination of alleles is present, and also possible that the disease is causally heterogeneous so that some -- even most - cases of the disease are produced by nongenetic causes.

Corresponding to genetic diseases of this type are genetic disease susceptibilities. These are instances in which the presence of a combination of alleles produces, in standard environments, an increased probability of acquiring a disease, and in which we do not know how to partition the population further to recognize other genetic or environmental determinants.

The second type of genetic disease comes about through modification of DNA in somatic cell division. Here the disease phenotype is brought about, in all standard environments, by the presence of some collection of somatic cells with modified DNA. Well known instances of cancer show that there are genetic diseases of the second type 
for which there are genetic disease susceptibilities of the first type: people with certain genotypes have increased probability, in all standard environments, of developing a collection of somatic cells that will produce disease. However, just as clearly, environmentally induced instances of cancer show that genetic disease susceptibilities of the first type need not be involved in the acquisition of a genetic disease of the second type.

The potential for confusion should be obvious. Characterizing cancer as "a genetic disease" may mean any of several things, and that characterization is quite consistent with the idea that many cancers come about as the result of environmental causes. Molecular geneticists are familiar with these distinctions, and are not usually swept into fallacious inferences. Outside of molecular genetics, though, the widespread use of the idioms discussed above can lead to dangerous misapprehensions. Turning away from disease to human behavior, consider, for example, the idea that there is a "gene for homosexuality". For reasons that will soon be apparent, it will be very difficult to establish claims like this one. Yet, even if some study of twins reared apart were to show that there is a locus at which a particular combination of alleles is associated with same-sex preference across a broad range of environments, it would still be possible (even probable) that the phenotype -- preference for sexual partners of the same sex -- is causally heterogeneous, with different combinations of genetic and environmental factors operating in different cases. Moreover, it might still be true that the overwhelming majority of cases of homosexuality are brought about primarily by environmental factors. Finally, it could well be the case that even when the putative alleles are present, there are environments in which the phenotype does not result. At present, the gay community is divided on whether the conclusion that "homosexuality is genetic" will result in more enlightened attitudes towards homosexuals. Without regard to the social benefits or damage of widespread acceptance of that conclusion, it is important to realize that the claim that "there is a gene for homosexuality" does not 
imply that "homosexuality is genetic" in the sense that all homosexuals bear genes that cause them, in all environments, to prefer members of their own sex. The question "Is sexual orientation genetic or environmental?" has already taken a false step, assuming that the answer cannot be "Sexual orientation is many things, with some atypical cases falling into the extreme categories".

During the twentieth century there have been numerous attempts to reach conclusions about the causes of human behavior, especially those forms of behavior that are most fascinating to us. People cannot be manipulated like other organisms, and the classic methods of behavioral genetics have to work with "natural experiments", situations in which the effects of similar genotypes can be observed in different environments. Monozygotic twins reared apart offer the most direct evidence of how similarities or differences in phenotype depend, for a shared genotype, on the differences in the environments that the twins experience. Yet, even when these studies show a clear correlation it is always important to ask two questions: (i) how much of the space of environmental variation has actually been explored?; (ii) has the phenotype been misdescribed, so that we are really viewing a correlation between genotype and some other property which is associated, given features common to the two environments, with what we take to be the phenotype?

The HGP might advance the understanding of the genetics of human behavior in one of two ways. The first method would be to use the information generated in the mapping project, specifically the positioned genetic markers, to track potential behavior genes in families. However, this is subject both to confounding environmental effects and to difficulties in identifying genes when many genes can affect a phenotype (polygenic conditions). The latter problems stem from the obvious possibility that a marker tightly linked to one pertinent gene may be only loosely linked to another, so that it becomes difficult to distinguish between genetic heterogeneity (different cases are caused in different ways) and loose linkage between a marker and a single locus. 
Analysis of polygenic physiological conditions -- in which the potential for environmental confounding is greatly diminished - has proved difficult enough (Lander and Botstein 1986). It is highly unlikely that the sophisticated results of human genome mapping will greatly refine the existing methods of behavioral genetics.

The classical methods try to understand the association between genotype, environment, and phenotype by focusing on the termini, not on the developmental processes that connect them. In principle, molecular biology might pursue the strategy of probing those processes, understanding the limits that our genes may place on our behavior by recognizing how parts of our neural machinery develop, and how they interact with the physical and social environment. To the extent to which molecular biology can lead to advances in neuroscience, there may be breakthroughs in understanding the genetics of human behavior.

In the beginning, it is likely that molecular accounts of the springs of behavior will be developed by turning to animal models. Research is already underway to try to fathom simple neural mechanisms and their relations to genotypes. Predictably, this research is beginning with the most elementary kinds of systems -- such as rudimentary forms of memory in flies (Tully 1991, 94) -- that are at a far remove from the traits that excite public attention. However, instead of an impetuous program that will yield controversial conclusions about aggression, sexual preference, and the other forms of behavior that most interest us, public interest will almost certainly be better served by the careful and painstaking study of simple systems that are likely to be conserved in the history of life. Our best chance of avoiding the well-known difficulties of disentangling the contributions of genetic and environmental causes of behavior is to proceed by linking molecular genetics to developmental neurophysiology and to see how far this approach will take us.

During the past decades premature conclusions about the genetic determination of behavior have played an enormous role in labelling, discrimination, and the 
infliction of suffering. The large literature on the foibles of biological theorizing about human behavior draws its moral force from two sources: the tenuous evidence for the conclusions reached (bom, largely, of the difficulty of obtaining firm results from the classical methods of behavioral genetics), and the grave consequences of wrong decisions. Some critics of the HGP believe that the project will encourage a renewal of harmful forms of genetic determinism. That need not be the case, if the uses of human genome mapping within behavior genetics are thoroughly scrutinized, if the primary approach to the genetics of behavior takes the more cautious route of neuroscientific studies of nonhuman organisms, and, above all, if the distinctions drawn in the foregoing paragraphs are widely understood. Public understanding of talk about genes is vulnerable to confuse those distinctions -- molecular geneticists find them elementary, but they are quite foreign to the nonspecialist - and it would probably be better if the "gene for" locution were banned from the media. Improved education in genetics will surely help, but constant vigilance will probably be required if we are to avoid the costly social and public policy mistakes that have been made in the twentieth century. In affirming the decision to sterilize Carrie Buck because she allegedly bore a gene for feeblemindeness, Oliver Wendell Holmes remarked that "Three generations of imbeciles is enough!" Not only was the genetics incorrect, but the casual acceptance of determinist claims led to a series of injustices (Gould 1981, Reilly 1991). A century of confusions about genetic determinism has surely been enough.

\subsection{Eugenics}

There is widespread fear that the HGP will foster a revival of eugenics (Duster 1990). The history of eugenics in the USA presents a series of episodes, some absurd (the awarding of certificates and prizes to outstanding examples of human stock at State and County Fairs), others deeply tragic (the involuntary sterilization of the "feebleminded", the refusal of entry to the US of people of "inferior genetic quality" 
who would be returned to Nazi-dominated areas of Europe -- see (Kevles 1985)). Of course, Nazi ventures in eugenics were even more horrific, and it is not hard to understand why a connection between the HGP and eugenics is perceived as a reason for opposing genomic research.

Does any such connection exist? Before that question can be answered it is important to become clearer about what is meant by "eugenics". Diane Paul has cogently argued that there are many different types of programs that have gone under the label of eugenics (Paul 1992). To appraise the HGP, we must ask what kinds of eugenic programs, if any, it might foster, and whether these eugenic programs are socially harmful.

Eugenics can be understood as either a theoretical discipline or as a practice based upon that theoretical discipline. The central problem of theoretical eugenics is to understand how patterns of reproduction affect the bringing into being of valuable populations of people. This theoretical study might be criticized on a number of grounds. Two criticisms are especially important. One denies that there is any objective, or even widely-shared conception of the values of people's lives, so that any such study is inevitably a mixture of reputable science (at best -- and the history of eugenics supplies numerous examples in which the claims about genes have been very poorly supported) with the particular ethical ideas of the group engaged in theoretical eugenics. The second protests the exploration of consequences with attention to just one kind of causal factor - focusing on the ways in which differential transmission of genes brings about populations with particular characteristics -- instead of thinking about how modifications of the environment might lead to valuable consequences.

Individual values should not be imposed as if they were universal. Nevertheless there are surely characteristics which, everyone would agree, diminish the lives of those who bear them; conversely, there are traits that would be unanimously conceded to enhance the quality of life of their bearers. Among the former 
characteristics we would be able to include the most severe genetic diseases -- TaySachs, Lesch-Nyhan syndrome, perhaps cystic fibrosis. Among the latter we would be able to count increased resistance to debilitating disease.

Similarly, the serious study of how to make the lives of our descendants more valuable should embrace both the possibility of acting to affect which alleles are transmitted and the possibility of modifying the environments in which they will live. Eugenics would thus be transformed into a wide-ranging study of the consequences of reproductive decisions and of other forms of present action with a view to understanding how these will affect the quality of the lives of future people, where quality is assessed by drawing on widely-shared views about how individual lives would be enhanced or stunted. Far from being socially noxious, we might view this study as a necessary human responsibility.

Major worries about eugenics surely center on its practical role, not on its cogency as a theoretical discipline. When theoretical eugenics has been translated into social action, the application has involved four important foci of decision:

1 - the population within which the practice is implemented

2 - the agents who are to make reproductive decisions

3 - the kinds of qualities that are valued or rated negatively

4 -- the status of the information used to draw conclusions about reproductive consequences.

Much criticism of past eugenic practices implicitly centers on the last type of consideration. It is now apparent that many of the genetic hypotheses that were used to make decisions about sterilization or to refuse immigration to the members of particular ethnic groups were not only false but accepted on the basis of flimsy evidence (Kevles 1985, Lerner 1992, Gould 1981). Yet the failures of past "genetic knowledge" are by no means the only troubles of the eugenic practices: one kind of objection to sterilizing the inmates of asylums decries the injustice done to those (like 
Carrie Buck and her sister) who were misclassified; a different criticism protests the interventions whether or not the scientific basis is accurate.

The genetic information emerging within contemporary molecular biology is doubtless far firmer than that applied in the heyday of American eugenics. Much is known about the genes involved as causal factors in many kinds of physiological conditions, and the HGP will vastly increase this knowledge. However, as noted in $\$ 8.2$, it is important to beware of overestimating the evidence available for claims about the causes of behavior. With respect to the traits that are most complex, the methods of genetics are not much different from those employed in the earlier history of eugenics, and the dangers of misclassification persist. Unless practical eugenics starts from a sober awareness of the uncertainties attending studies of the genetics of complex human behavior, it is bound to be irresponsible.

In the past, damaging eugenic programs have singled out particular populations for intervention: Nazi programs focused, for example, on Jews, Gypsies, and homosexuals. These programs have also coerced members of these populations into making reproductive decisions that accord with centrally-imposed policies. At present, the HGP and the practice of US medicine make no overt identification of a population within which reproductive decisions are to be changed and offer no explicit policy for changing those decisions. The most obvious consequence of an increase in molecular genetic information and the consequent proliferation of pre-natal testing would be a type of laissez-faire eugenics, in which individual US citizens make their own reproductive decisions, in the light of increased genetic knowledge and their own standards of what is valuable, and these decisions would have an aggregate effect on the genetic composition of later populations. However, those concerned that the HGP will foster a new eugenics are, almost certainly, less worried about this laissez-faire program than with the idea that inequalities of access to genetic information will effectively localize the practice to certain subpopulations and that widespread attitudes 
towards some traits (including unwillingness to support children who suffer some disabilities) may effectively coerce some reproductive decisions.

The singling out of particular groups and the overriding of individual values are disturbing features of the eugenic practices of the past, and it is important to recognize that they may arise again when the knowledge resulting from the HGP is applied in US society. It is too simple to believe that if there are no governmental restrictions on the use of reproductive technologies (diagnostic tests, IVF etc), that prospective parents will be free to make the reproductive decisions they want. The costs of these technologies may provide an equally effective barrier, leading to a situation in which children whose genotypes place them in most need of environmental support are born, in disproportionate numbers, into strata of society that are least able to provide such support. Furthermore, even though prospective parents may be prepared to value and nurture a child with a genetic condition, even though they know that that condition always produces a phenotype that is not valued within US society, they may reluctantly decide to terminate a pregnancy when the genetic condition is present in the fetus, on the grounds that, for all the efforts they might make, the resulting child would have to suffer too much (Powledge 1988). In such easily envisageable cases, the actions do not reflect what the prospective parents most value, and their choices must be viewed as partially coerced (Katz Rothman 1986, Rapp 1988).

Without a broad attitude of respect for difference, and a determination to enable each child, adolescent, and adult to develop maximally, individual reproductive decisions will inevitably be affected -- possibly even partially coerced --by social preferences for certain types of people. If the transition from eugenics to genetic counselling (or to "laissez faire eugenics") is to eliminate the tendency of old practices that is most troubling -- the tendency to try to transform the population in a particular direction, not to avoid suffering, but to reflect a set of social values -- then it will be necessary to combine the attempt to bring into the world children with high chances of 
lives of high quality with a public commitment to realize lives of the highest quality for all those who are born. A maximally free reproductive decision would be one in which the prospective parents knew

(a) how the genetic condition of the fetus would affect the maximal quality of life of the person who would be born

(b) that the society in which that person would live would be committed to maximizing the quality of life for all people born into it, and that all such people would be given respect.

Under these conditions, prospective parents could make a decision based on their assessment of the value of a potential child's life, and their understanding of their own responsibilities in making demands on society.

The previous discussion shows that the kind of "eugenics" that might result from the HGP could avoid those features that make the eugenic practices of the past so repellent. Utopian eugenics would incorporate:

(i) reliable genetic information

(ii) equal access to that information and to applications of it

(iii) wide public discussion of values but no socially-imposed restrictions on reproductive decisions

(iv) widely-shared respect for difference, coupled with a public commitment to realizing the potential of all those born.

When champions of the HGP contemplate the consequences of increased genetic knowledge for reproductive decision-making, they seem to believe that the only kind of eugenics that will result will be utopian eugenics, and they may hold, with some plausibility, that this is unproblematic. (It may well be counted as beneficial, in that births that would have brought great suffering will be avoided.) Critics fear that a far less benign form of eugenics will result, and this fear can readily be understood by recognizing how unlikely it is that the conditions (ii) and (iv) will be realized within US 
society in the immediate future. While there may be no reason to distrust the eugenic consequences of the HGP in principle, the actual results in practice may be far more disturbing.

Once the notion of utopian eugenics has been recognized, it is necessary to face the possibility that it does not place sufficient constraints on individual reproductive freedom. The classic motivations for eugenic programs involve the idea that individual choices may lead to costs that a society cannot bear. Such arguments may be sharpened by taking seriously the commitment to allow those who are born to develop in ways that will give their lives maximal quality. Combining this commitment with the idea of honoring individual reproductive freedom raises the specter of a situation in which unrestrained decisions to procreate lead to the birth of people whose lives cannot be sustained to anything approaching maximal quality without stretching the resources of society to breaking point. The classical eugenicists worried that undirected reproductive practices would so increase the "genetic load" of a population that, eventually, the future of the species (or of particular "races") would be imperilled (Kevles 1985). In the present version, the concern is that, without a socially directed reproductive policy, the commitment to doing what can be done to develop individual lives will consume so many social resources that economic growth (and other forms of healthy national development) will become impossible.

Is it possible to resist either limiting individual reproductive freedom or weakening the commitment to provide for those born with disabilities, who require social support if they are to live valuable lives? The conflict between these two attractive ideals comes about because individual reproductive decisions, taken in isolation, may add up to social disaster. That would be avoided if reproductive decisions were taken with an eye to social consequences, if prospective parents were not only to think about their own wishes but also about the consequences of giving birth to children who will require social support. Few people presently take this perspective 
in planning their families, and it may be thought that conflict between reproductive freedom and the commitment to public support is inevitable for any (or all) the following reasons:

A. Some (most?, virtually all?), reproductive decision-making is socially irresponsible

B. The social pressures on individuals to make problematic reproductive decisions are too strong

C. For some people, even the best reproductive decisions are likely to make demands on others.

Now it is surely true that much reproductive decision-making at present is unplanned, and virtually all is taken in ignorance that might be ameliorated by advances in molecular genetics and pre-natal testing. However, it is surely possible that, without a centrally-imposed eugenic policy, there can be public education about the consequences of bearing children with particular genetic conditions. People can be informed about what kinds of lives those who bear particular alleles will lead, given a diversity of possible environments, and about the costs to society of providing those environments that allow for lives of the highest quality. Educational programs might attempt to develop commitments to reproductive responsibility. It is probable that there will always be instances of type $C$, cases in which a couple can only avoid a high risk of giving birth to a baby who will make large demands on society by forgoing reproduction entirely. With greater emphasis on the value of social parenting, particularly an attitude that sees adoptive parents as full ("real") parents, not to be distinguished from biological parents, perhaps it would be possible to reduce the frequency of such cases to a level at which it could easily be absorbed.

There remains the worry that if an educational program is to be effective, it will become an instrument of coercion that constrains the choices of citizens. That worry is quite reasonable if the program takes the form of an ideology, reflecting the values 
of some privileged class. Yet it is by no means inevitable that this should be so. The goal is to develop an educational program that genuinely enhances the freedom of those who make reproductive choices. To attain this goal it will be important to promote: (a) clear public understanding of the social consequences of individual reproductive decisions, (b) close scrutiny of the information used in generating those consequences, (c) close criticism of background social assumptions about the values of the lives led by particular types of people, and (d) public debate about ways of improving the quality of individual lives.

The blanket charge that current research in molecular genetics will revive eugenics is too simple, but, as this section has revealed, there are considerable dangers that the admirable attempts to try to use molecular biology to avoid lives of inevitable suffering or of pinched opportunities will be degraded in practice to take on some of the troublesome features of the eugenic movements of the past (Duster 1990, Hubbard and Wald 1993). Resisting the process of degradation will require serious thought about the issues raised here and ample public discussion of issues that most people would prefer to avoid.

\subsection{Abortion and Genetic Testing}

One question that is not widely discussed in conjunction with the HGP is the role that abortion is likely to play, in the foreseeable future, in individual reproductive decisions. Within a decade there will be an extremely large number of genetic tests that can be used, in conjunction with amniocentesis or chorion villi sampling, to detect the presence of various conditions in a fetus. Assuming that the introduction of these tests is accompanied by collecting statistics that reveal clearly the connections among genotype, environment, and phenotype, prospective parents will be able to discover whether a fetus is at high risk for a variety of painful or limiting diseases. Unless therapeutic options become available at a much faster rate than can now be predicted, 
this knowledge will only be useful insofar as it enables families to prepare for the birth of a child with special needs or as it leads them to terminate pregnancies that would otherwise have been continued. Past studies of pre-natal testing have shown that a significant number of women who learn that the fetus they are carrying has a genetic disability do not decide to abort. Nonetheless, it is reasonable to expect that the growth in the number of pre-natal tests will increase the frequency of abortions. There will be a consequent lowering of the rate of birth defects (although it is important to remember that some genetically-based conditions are likely to remain undetected, that some birth defects are caused by intra-uterine events, and that a significant number result from birth trauma), and this will be beneficial both in the diminution of expenses of care (but see Technical Note 2 to Section 4) and, far more significantly, in the avoidance of suffering. It is important to remember that the benefits are not without cost, and that, for almost all women, the decision to terminate a pregnancy brings great distress (Katz Rothman 1986).

Nevertheless, many people, including those who have designed and championed the HGP, are convinced that the overall consequences of increased use of pre-natal genetic testing are good. They are committed to something like the following principle:

(P) A society in which fewer children with genetic disabilities are born as the result of pre-natal testing and selective abortion is better than one with a higher rate of birth defects.

There are three objections to $(\mathbf{P})$ which deserve discussion. The first offers blanket opposition to the use of abortion as a tool of reproductive planning. The second claims that the idea of planning reproduction at all violates something important about human life, birth, and the relation of mother to child. The third suggests that initial introduction of genetic testing to avoid diseases and disabilities will inevitably lead further to a greater incidence of morally dubious abortions. 
The complex question of abortion ranges far beyond the scope of the present report. Plainly, from some religious perspectives, the idea of pre-natal testing and abortion involves both an arrogant questioning of God's design and the murder of a person. A complete response to these charges would be very lengthy. Nevertheless, three points need to be recognized. First, there are many occasions on which we intervene in nature in the interests of promoting what we regard as valuable human lives. Few people are inclined to suggest that our efforts to stamp out the infectious agents responsible for much disease and death violate God's design. Religions rarely require that we take life completely as it comes. Hence the special difficulty with abortion cannot be that we are arrogantly intervening with a life that God has designed, but that we are ending a potentially valuable human life. The first charge thus reduces to the second.

The next point to be appreciated is that decisions not to terminate a pregnancy can often appear extraordinarily cruel. There are some diagnosable genetic conditions (Tay-Sachs, San Filippo Syndrome, Lesch-Nyhan) which bring suffering to their bearers and to those who will care for them. Pre-natal testing would enable these conditions to be identified before the stage at which the fetus develops the neuronal connections that make possible sensations of pain. Abortions could thus be carried out to avoid enormous anguish, and to deny the legitimacy of doing so by appealing to abstract religious doctrine -- especially when texts are amenable to a variety of interpretations -- seems insensitive.

Third, and most important, is an approach to the abortion controversy that has been articulated by Ronald Dworkin (Dworkin 1993). Although it is common for the debate to be framed in terms of the question "Is the fetus a person?", Dworkin argues that this is neither correct nor in accord with the deepest views of the contending parties. On the first score, he suggests that a collection of cells, incapable of sentience, should not be regarded as a person. On the second, he proposes that the 
conflict about abortion results because of a dispute about the sources of value of human lives. Those who would sharply limit the conditions for abortion (or, perhaps, even ban abortion altogether) believe that a human life is valuable -- "sacred" -- simply in virtue of its conception as a human life. Liberals about abortion view the value (sacredness) of human lives as arising differently, as the result of human investment in those lives. They can thus regard the decision to continue some pregnancies as greatly diminishing the values of lives -- the lives of the parents, of children and other relatives -- in which there has already been a substantial human investment, and to see this diminution of value as a far greater loss than the cessation of a human life that has not yet "begun in earnest".

Dworkin's framework is helpful, not least because it enables us to appreciate why some situations may involve anguish and a sense of loss, whatever is done. If it succeeds in liberating us from the idea that fetuses are persons, it will also undercut the second major charge against the use of abortion in conjunction with pre-natal testing. There would no longer be any recourse to the notion that terminating a pregnancy is killing a person, or a debate about the clash of rights between mother and fetus. Instead, it would be required of us to think about how continuing or terminating a pregnancy will diminish the value of the lives of those affected. In instances in which the fetus bears a severely debilitating genetic condition, not only is the human investment in it small but it has little potential for continuing a life of high quality. Unless great emphasis is placed on the value of fetal life simply because it is a human life, the loss of value incurred by continuing the pregnancy is thus likely to be very much larger than that brought about by termination. Opposition to the use of abortion must come to rest on the principle that any life that is the product of human conception is so valuable that it would be wrong to end it, and it is not easy to see how to sustain so abstract a principle in the face of the suffering brought about by the birth of children whose nervous systems will degenerate in early infancy. 
It is worth re-emphasizing that any discussion of questions of abortion here is incomplete. However, issues about abortion cannot be ignored in discussing the implications of contemporary molecular biology. Foes of abortion should view prenatal testing as paving the way for evil, and it is important for any defense of the HGP to respond (if only briefly) to their concerns.

The second major objection to $(\mathbf{P})$ is often expressed as the charge that contemporary reproductive medicine has reduced life to another commodity, and has brought us to a position in which babies are regarded as products subject to assessment and quality control (Katz Rothman 1986). This criticism rests, in part, on the sense that gynecological practice is often dehumanizing, and it is quite reasonable to demand that doctors and hospitals increase their attempts to combine care of mother and fetus with respect for those aspects of pregnancy and birth which give these processes special significance for us. Pre-natal testing might easily take place in settings that deny that respect, by thinking of the value of a fetus in debased ways -- for example, by attending only to the socially-valued achievements that the person who would develop from that fetus is likely to enjoy. Discussions of the value of genetic testing, both in section 4 and in the present section, suggest a different conception, one that avoids thinking about the "worth of the product" in terms of potential accomplishments, but that finds a place for reflection on the value or quality of human lives. Without treating babies or children as commodities, it is possible to recognize that their lives differ in quality, that a child who will always be confined to a hospital bed will live a life of lower quality than one who can engage in a broad range of activities, that a child with compulsive tendencies to self-mutilation will live a life that is more painful than one who lacks these propensities. Not all valuations of lives adopt the perspective of outsiders who place great emphasis on certain types of accomplishments; it is possible to recognize that different people will have very different senses of themselves and of 
what is central and important to their lives, while distinguishing some lives as being of lower quality.

Judgments about the quality of individual lives can easily seem elitist, but contemporary moral philosophy provides a useful framework for making such judgments while honoring individual differences (Dworkin 1993, and, especially, Brock 1993). There are a number of separate dimensions for thinking about how well someone's life has gone -- or, in the cases of most concern here, how well it is likely to go. One has to do with experiences of pleasure and the avoidance of pain. A second has to do with being able to form a sense of one's own life, to decide what is important for oneself, what one wants to do, what kind of person one wants to be (Daniels 1985 33). For some people, the choices for this type of life planning and for translating their plans into action are much more limited, and, on this dimension, these unfortunate people live lives of lower quality. Third, and finally, the development of a plan for one's life generates desires and wants that are central to that life, and, on the third dimension, lives go more or less well according to whether these desires are satisfied or frustrated.

There are some genetic conditions which can be identified before birth that make it impossible for their bearers to live lives of even modest quality. We know of no ways of modifying the environment to enable children who bear these conditions to avoid severe problems along one of the three dimensions just distinguished. In some cases, as with various types of juvenile arthritis, it is impossible to avoid excruciating pain. In other instances - Tay-Sachs, Lesch-Nyhan, and other examples that have figured above - the opportunities for any type of self-development are appallingly restricted. To judge that children with the pertinent genotypes are doomed to lives of poor quality is not to engage in "commoditizing life", but rather to appreciate what is most valuable about human life and to treat sympathetically and mercifully those who lack the preconditions for living human lives that attain genuine value. 
There are two subsidiary arguments that underlie the attack on pre-natal testing as quality control. One insists that it is impossible to combine the attitude of seeking to prevent the birth of people with particular conditions with respect for those who are fortuitously born with those conditions. The second decries the idea of reproductive planning on the grounds that it represents an overly prudent approach to human life.

Many people who are at most risk of conceiving a child with a genetic disease or disability are reluctant to take prenatal tests for the condition (sometimes, after taking such tests, they are reluctant to consider terminating a pregnancy) because they have a relative, whom they love, who already has the condition. They regard the possibility of terminating a pregnancy, because the condition is present, as excluded, on the grounds that abortion would express a devaluation of someone they love: parents sometimes ask "How could I explain to my daughter (son) that I had had an abortion because of just the condition she (he) has?" This poignant question is entirely natural, but it overlooks a moral perspective which would resolve the tension that such parents no doubt feel.

There is a great difference between contemplating a life in prospect when there is, as yet, no person, no subject of pain and (usually, though, tragically, not always) no subject of conscious experience, and interacting with a person already brought into being. However limited the opportunities for a child may be, and however terrible their suffering, it is required of us to do the best we can to enhance the quality of the child's life and to show the respect that is due to all persons. Recognizing this moral demand is perfectly consistent with believing that it would be better for the life of a fetus with the same genetic condition not to be continued, for, in this instance, there is not yet a person to serve as the source of the moral demand. In prospect, we do what we can to avoid bringing into being human lives whose quality will inevitably be diminished. In retrospect, we should respect and love those whose lives are similarly 
bounded, and we should do what we can to ensure that their lives have the highest possible quality.

Although this is a consistent attitude, it may seem far too abstract as a response to the question "Would you decide to terminate a life like mine?", uttered -- or imagined to be uttered -- by a child whom the parents love. But a parental decision to terminate a pregnancy when the fetus is found to have a similar genetic condition should not be understood as a rejection of the child. If the parents are already doing what they can to enhance the child's quality of life, then the decision to abort the fetus should be viewed as an expression of their sense of inadequacy in being unable to do more, a sympathetic recognition that, for all their efforts, there is still considerable suffering or the opportunities for self-development are still constricted.

The last version of the argument that pre-natal testing treats human lives as commodities rejects the idea of planning lives (Ruth Hubbard, personal communication). It is quite correct to point out that no amount of pre-natal testing will guarantee that a couple has children who are assured health and happiness. Yet this is to mistake the point of pre-natal testing. Those who decide, almost always with distress, that they should terminate a pregnancy in which the fetus has been diagnosed as bearing the allele for Tay-Sachs, Lesch-Nyhan or myotonic dystrophy are not seeking guarantees that they will have only children whose lives will flourish. Instead they are trying to avoid situations in which the quality of the lives of their children is inevitably diminished. Pre-natal testing does not involve a pathological attempt to evade risks: like many actions that parents take to protect the lives of children preventing them from playing in busy streets, warning them against addictive drugs, and so forth -- there is no illusion that all contingencies can be anticipated and met. Unexpected dangers and reversals are always possible, but this hardly means that we should not take steps to guard against situations that would surely bring suffering. 
The final objection to $(\mathbf{P})$ questions the possibility of restricting abortion to those cases in which the rationale in terms of the quality of lives is clear. Many thoughtful critics of the use of abortion in conjunction with pre-natal testing are prepared to acknowledge the positive effects of lowering the incidence of the most devastating diseases and disabilities, but they fear that the HGP will make available a large body of genetic tests that will draw us, ineluctably, into terminating pregnancies on grounds that are far less justifiable. Tay-Sachs and Lesch-Nyhan may represent clear cases, Fragile X Syndrome and CF are somewhat less clear, juvenile diabetes more problematic, and, if genes for these traits were discovered, homosexuality, low I.Q., and obesity would engender very serious concerns about the morality of abortion. How can a line be drawn to separate the permissible cases from those that are morally repugnant?

The question can be addressed either at a practical level, asking whether we can expect a proliferation of genetic tests to drive us down a slippery slope, or in moral terms, asking whether there is a principled distinction that can be drawn. As noted in section 6, gene replacement therapy is, at present, far too crude to enable people to do much more than attempt to avoid the most crippling conditions among their offspring. Selective termination by abortion is almost as blunt an instrument for fashioning the traits that parents might want their children to have. Even setting on one side the research showing that mothers typically find decisions about abortion to be personally wrenching, and that their dominant concerns are with the health of the babies whom they will bring into being, it is important to appreciate that even a thoroughly callous couple, determined to have only babies meeting morally frivolous standards of perfection, would be extremely limited in their ability to realize their goals. A woman carries roughly five hundred eggs. Taking into account the time needed for conception and bringing the fetus to a stage at which genetic testing is possible, a decision to terminate a pregnancy consumes at least five (and quite possibly a much larger 
number) of a maximum of five hundred opportunities for pregnancy. Knowing that the risks of chromosomal aberrations increase with advancing age, it would be foolish and imprudent for a woman in her twenties to abort a fetus lacking any genetic conditions associated with disease or disability, on the grounds that the fetus carried an allele indicative of a trait -- obesity, low I.Q., same-sex preference -- that the woman viewed as incompatible with her ideal. (Women who made judgments in this way would be likely to produce rather few, if any, children - and, perhaps, given the character of their decision-making, this is just as well.) Selective abortion is a way for women to raise the probabilities that their children will be healthy; it is not a rational method for designing "perfect babies". Combining this conclusion with the recognition that the overwhelming concern expressed by most women is that their babies not have the most devastating forms of disease, it is easy to see that the vision of a slippery slope conjures up a wildly implausible picture of pre-natal decision-making.

Furthermore, it is far less difficult to draw moral distinctions among cases than might initially appear. What is most repugnant about the idea of using abortion to design the characteristics of children is the imposition of a system of values that is quite external to the child's life: parents try to shape children according to their image of perfection, not recognizing that children who did not conform to that image might have valuable lives. The response to earlier objections conceived pre-natal decisions as governed by an attempt to ensure that the quality of life of those born was not seriously diminished. If prospective parents employ this perspective in considering whether or not to terminate a pregnancy, then the moral status of their decisions will look very different. Most often, they will judge that the fact that a child bears a genetic predisposition to low I.Q., obesity, or homosexuality (assuming, contrary to the points made previously in this report, that genes for these traits are likely to be identified) does not inevitably diminish the quality of his/her life. In these instances, it is usually possible to enable the child to engage in processes of self-development that will bring 
genuine satisfaction, although, depending on the environment in which the child will live, this may require more or less work to overcome social disapprobation, prejudice or intolerance. In some extreme instances, the parents may judge that there is no way for them to rear a child in a society that will permit significant self-development: the segment of society in which they find themselves may be so hostile to those with particular traits that the problems the child faces will be insuperable. If, on reflection, they decide to terminate the pregnancy because they feel the child who would be born would be doomed to be unhappy, that decision should not be condemned as morally wrong. The moral problem attaches to the social prejudice.

Intermediate cases show how thinking in terms of potential quality of life makes principled moral decisions possible. At present, the prospects for children with $\mathrm{CF}$ are genuinely mixed, not simply because of the variable expression of the disease, but also because of the differences in resources that parents may bring. Assuming that the severity of the condition becomes more readily predictable, we can imagine parents assessing their own ability to overcome the factors that would otherwise decrease their child's quality of life. Some parents, recognizing their own psychological and economic resources, as well as their relative freedom from competing obligations, could rightly decide that they would be able to ensure a high quality of life for a child with $\mathrm{CF}$, choosing to continue the pregnancy. Those situated differently might recognize that they could not overcome the genetic obstacles, and, regretfully, decide on abortion. Such responsible decisions contrast with cases in which parents who have the resources to cope elect to terminate (say on the grounds that they don't want to invest in an "imperfect" child) or in which parents thoughtlessly bring into the world a child whose life, in the environment they can provide, is inevitably miserable.

The "slippery slope" argument -- "if we start aborting fetuses with devastating diseases, we'll end up aborting on the basis of traits that groups in society don't like" -can be resisted, both by making clear its distance from actual and rational pre-natal 
decision-making and by showing how the ideal of ensuring the possibility of lives of quality for those who are born makes principled distinctions. If pre-natal decisions are thoroughly influenced by this ideal, and there is some evidence that pregnant women already think in these terms, then fetal testing and selective termination of pregnancies can play a morally valuable role in influencing the lives that our descendants lead.

\subsection{Priorities}

If bio-medical research builds on the findings of the HGP, in the ways envisaged in earlier sections, then it is possible that, in several decades, medical practice could look very different. Assuming that enough is known about the causal pathways underlying various diseases to intervene early in the prevention of those diseases -- as, for example, may become possible quite quickly with colon cancer -- it will be important to make genetic tests widely available, and to provide, for those who are at high risk, the means of avoiding the diseases. In effect, there will be a change of emphasis from reactive medicine (attempting to undo previous damage) to preventative medicine (attempting to forestall damage).

The economic consequences of this shift will need careful exploration. If greatly increased efforts at prevention of disease are simply combined with current commitments to prolong patients' lives as long as possible, then it appears overwhelmingly likely that the expense of medicine will grow: genetic tests, subsequent monitoring, and early interventions will all involve costs; although patients may live longer, healthier, lives as a result, they are likely, in the end, to reach states in which they can only be kept alive by costly care; without a change in medical commitment, we can expect that providing that care will be roughly as expensive as it is at present.

If this impressionistic argument is borne out by a more detailed economic analysis, there will be a number of options. It would be possible to leave medical 
practice much as it is, and not to deploy the resources that the HGP makes possible; alternatively, we could bear the extra costs, recognizing the benefits in terms of longevity and improved health as outweighing the expenses; finally, we could change our attitude towards the goals of medicine, abandoning the notion that it is a medical duty to prolong life to its utmost, in favor of the idea that people's lives are most enriched by taking steps early to guard them against disease and disability.

In recent years, there has been some discussion of the fact that large amounts of money (roughly $85 \%$ of the costs of US medicine) are spent in prolonging lives for rather short periods (approximately 18 months) -- see (Menzel 1983). Many doctors plainly feel an obligation to save their patients' lives, even when the extra period they secure only allows for low quality of life. Yet, if forced to choose between resources used early, to prevent damage to the cardiovascular system for example, and resources used late, such as cardiac surgery after a major heart attack, most of us would surely prefer the former option. That preference seems entirely reasonable. Early investment of resources may provide us with 85 years of healthy life, and, even though we give up the opportunity for some heroic surgery in our years of decline, that surgery would only have offered us an extra year of diminished life. If medicine guaranteed us 85 years and a quick exit, then we would surely reject the offer of 75 years and an extra year of lingering. Of course, medicine offers no such guarantee, nor is it likely to do so, but the probabilistic expectations, given a fully developed preventative medicine versus the reactive practice of the present, are likely to stand in something like these relations.

If the costs of simply adding tests, monitoring and preventative interventions to current reactive practice turn out to be more than US society can bear, then the biomedical results that flow from the HGP are likely to force us to confront the choices of the last paragraph. We shall have to contemplate whether the goal of medicine inherited from the Ancient Greeks -- to promote health and to cure disease -- is no 
longer the proper expression of our ultimate aims in a changed scientific situation. An alternative conception of medicine, one that we might want to adopt, would see the discipline as one instrument (among others) for promoting the quality of individual lives, and to see this enterprise as best carried out by using resources early in lives (Daniels 1985, Menzel 1983).

Increased biomedical knowledge is likely to generate priority decisions within medicine. To transfer to medical practice all the potential tests, and to do so in ways that are both effective and fair, would involve an enormous expenditure of resources. (Section 4 attempted to underscore the need for statistical information and for greatly improved counselling.) If tests and preventative strategies are to be introduced, choices will have to be made. Some genetic conditions (genetic predispositions to various forms of cancer or to cardiovascular disease) affect a significant fraction of the US population; others (Fragile X Syndrome, Huntington's Disease) are quite rare but produce devastating effects when they are present. In light of the discussion of sections 4 and 6 , it will be important to scrutinize carefully the possibility of using genetically-based strategies to do significant good: application of findings about colon cancer may be more effective than corresponding applications with respect to breast cancer, early testing for CF may have more beneficial results than testing for Huntington's Disease. Pre-natal testing for the most severe inherited conditions can be expected to prove economically cheap (despite the emotional costs that it may bring in some instances). Yet it is possible that biomedical research generated from the HGP should be so successful that there will be genuinely hard choices. Should we use our resources to bring a significant benefit to those who suffer diseases that are quite common? Or should we first seek to relieve the few people on whom rare genetic diseases have devastating effects? A principle of maximizing total well-being might well favor the former course of action; a principle of attending first to the most urgent needs before treating the lesser needs of others would seem to favor the latter. 
Finally, it should be remembered that the HGP is not the project of a single nation. It is being pursued internationally, and it has the potential to affect the wellbeing of people all over the globe. As greater understanding of the molecular bases of disease becomes available, researchers in the affluent countries may face even broader questions of setting priorities. Malaria accounts for the deaths of millions of children annually. Although the treatment of infectious diseases may seem remote from the HGP, many researchers hope that increased molecular knowledge will lead to ways of disabling viruses, bacteria, and other infectious agents. To what extent should the biomedical research and the applications of research in the affluent world make the well-being of the citizens of that world the first priority? To what extent is there an obligation to develop medical responses to worldwide problems of disease? Lastly, as we consider the possibility that major types of disease might prove eliminable, how should we integrate the treatment of disease with appropriate measures for responding to the needs of a population that might then be expanding at an even faster rate?

These are enormous questions, and the HGP will not raise them immediately. Nevertheless, it seems appropriate to recognize that, if the program fulfils its promise, it will confront our descendants with further decisions of extraordinary difficulty. 


\section{REFERENCES}

Andrews, L.B. et.al. 1994 Assessing Genetic Risk, Washington D.C.: National Academy Press.

Angastiniotis, Michael et.al. 1986 "How thalassemia was controlled in Cyprus", World Health Forum, 7, 291-297.

Annas, George and Elias, Sherman 1992 Gene Mapping: Using Law and Ethics as Guides New York: Oxford.

Beckwith, J. and King, J. 1974 "The XYY Syndrome: A Dangerous Myth", New Scientist, 64, 474-476.

Beckwith, Jonathan 1993 "A historical view of social responsibility in genetics", BioScience, 43, 327-333.

Berg, Paul and Singer, Maxine 1992 Dealing with Genes Mill Valley, CA.: University Science Books

Brock, Dan W. $1993 \quad$ "Quality of life measures in health care and medical ethics", in Brock Life and Death, New York: Cambridge, 268-324.

Caskey, C. Thomas "DNA-Based Medicine: Prevention and Therapy", in (Kevles and Hood 1992) 112-135.

Cohen, D. et.al. 1993 "A first-generation physical map of the human genome", Nature, 366, 698-704. 
Connor, J.M. and Ferguson-Smith, M.A. 1993 Essential Medical Genetics (Fourth Edition) Oxford: Blackwell.

Cowan, Ruth Scwartz 1992 "Genetic Technology and Reproductive Choice: An Ethics for Autonomy", in (Kevles and Hood 1992) 244-263.

Daniels, Norman 1985 Just Health Care New York: Cambridge University Press.

Daniels, Norman forthcoming "The Genome Project, Individual Differences, and Just Health Care", typescript.

DOE/NIH 1990 Understanding our Genetic Inheritance. The U.S. Human Genome Project: The First Five Years FY 1991-1995.

Draper, Elaine 1991 Risky Business, New York, Cambridge University Press.

Duster, Troy $1990 \quad$ Backdoor to Eugenics New York: Routledge.

Dworkin, Ronald 1993 Life's Dominion New York: Knopf.

Elias, Sherman et.al. 1992 "Carrier Screening for Custic Fibrosis: A Case Study in Setting Standards of Medical Practice", in (Annas and Elias 1992) 186-202.

Friedmann, Theodore (ed.) 1991 Therapy for Genetic Disease, Oxford: Oxford University Press.

Gilbert, Walter "A Vision of the Grail" in (Kevles and Hood 1992) 83-97.

Gould, Stephen Jay 1981 The Mismeasure of Man, New York: Norton.

Hagerman, R. and Silverman, A. 1991 Fragile X Syndrome: Diagnosis, Treatment, and Research, Baltimore: Johns Hopkins. 
Harris, John 1992 Wonderwoman and Superman: The Ethics of Human Biotechnology, Oxford: Oxford University Press.

Hoffman Baruch, Elaine et.al. (eds.) 1988 Embryos, Ethics, and Women's Rights, New York: Harrington Park Press.

Holtzman, N.A. 1989 Proceed with Caution, Baltimore: Johns Hopkins.

Hubbard, Ruth and Wald, Elijah 1993 Exploding the Gene Myth, Boston: Beacon.

Jones, Kenneth 1988 Smith's Recognizable Patterns of Human Malformation (Fourth Edition) Philadelphia: W.B. Saunders.

Kevles, Daniel J. 1985 In the Name of Eugenics, New York: Knopf.

Kevles, Daniel J. and Hood, Leroy (eds.) 1992 The Code of Codes, Cambridge MA.: Harvard.

Khoury, M.J. et.al. 1993 Fundamentals of Genetic Epidemiology, New York: Oxford.

King, Mary-Claire et.al. 1993 "Inherited Breast and Ovarian Cancer: What are the Risks? What are the Choices?", Journal of the American Medical Association, 269, 1975-1980.

King, Patricia 1992 "The Past as Prologue: Race, Class, and Gene Discrimination", in (Annas and Elias 1992) 94-111.

Lander, E.S. and Botstein, D. 1986 "Mapping Complex Traits in Humans: New Methods Using a Complete RFLP Linkage Map", Cold Spring Harbor Symposia on Quantitative Biology, 51, 49-62. 
Lander, Eric 1992 "Science, Law, and the Ultimate Identifier", in (Kevles and Hood 1992) 191-210.

Lerner, Richard M. 1992 Final Solutions: Biology, Prejudice, and Genocide, University Park, PA.: Penn State Press.

Lewontin, R.C. 1992 Biology as Ideology New York: Norton.

Lewontin, R.C. and Hartl, Daniel 1991 "Population Genetics in Forensic DNA Typing", Science, 254, 1745-1750.

Lewontin, Richard, Rose, Steven and Kamin, Leon 1984 Not In Our Genes, New York: Pantheon.

McKusick, Victor (ed.) 1992 DNA Technology in Forensic Science, Washington, D.C.: National Academy Press.

Menzel, Paul 1983 Medical Costs, Moral Choices, New Haven: Yale University Press.

Moen, Joan L. et.al. 1977 "PKU as a factor in the development of self-esteem", Journal of Pediatrics, 90, 1027-1029.

Nelkin, Dorothy and Tancredi, Laurence 1989 Dangerous Diagnostics, New York: Basic Books.

Nichols, Eve 1988 Human Gene Therapy, Cambridge MA.: Harvard.

NIH/DOE 1993 Genetic Information and Health Insurance, Report of the Task Force on Genetic Information and Insurance, pre-publication typescript. 
Olson, Maynard 1992 Contributions to Roundtable Discussion on Sequencing cDNAs. Los Alamos Science Number 20, 137-139.

OTA 1990 Genetic Monitoring and Screening in the Workplace, Washington, D.C.: U.S. Government Printing Office.

OTA 1992 Cystic Fibrosis and DNA Tests: Implications of Carrier Screening, Washington D.C.: U.S. Government Printing Office.

Parkman, Robertson 1991 "Organ Transplantation as Therapy for Genetic Diseases", in (Friedmann 1991).

Paul, Diane 1991 Review of three popular books on the HGP, Science, 252, 142-3.

Paul, Diane 1992 "Eugenic Anxieties, Social Realities, and Political Choices", Social Research, 59, 663-683.

Powledge, T. 1990 in The Genome, Ethics, and The Law (Proceedings of AAASABA Conference), Washington D.C.:AAAS.

Powledge, Tabitha 1988 "Reproductive Technologies and the Bottom Line", in (Hoffman Baruch et.al. 1988), 203-209.

Rapp, Rayna 1988 "Moral Pioneers: Women, Men and Fetuses on a Frontier of Reproductive Technology", in (Hoffman Baruch et.al. 1988) 101-116.

Rapp, Rayna 1994 "Amniocentesis in Socio-cultural Perspective", Journal of Genetic Counselling, forthcoming.

Rawls, John 1971 A Theory of Justice, Cambridge MA.: Harvard.

Reilly, Philip 1991 The Surgical Solution, Baltimore: Johns Hopkins. 
Reiman, Jeffrey 1976 "Privacy, Intimacy, and Personhood", Philosophy and Public Affairs, 5, 26-44.

Scanlon, T.M. 1975 "Thomson on Privacy", Philosophy and Public Affairs, 4, 315322.

Science 1988. Science 240 , Number 4858 , contains a valuable collection of review articles on various models organisms and their virtues.

Steinberg, Mark et.al. 1993 Recombinant DNA Technology: Concepts and Biomedical Applications, Englewood Cliffs: Prentice-Hall.

Sterelny, Kim and Kitcher, Philip 1988 "The Return of the Gene", Journal of Philosophy, 85, 335-358.

Suzuki, David and Knudtson, Peter 1989 Genethics, Cambridge MA.: Harvard.

Tauber, Alfred and Sarkar, Sahotra 1992 "The Human Genome Project: Has Blind Reductionism Gone Too Far?", Perspectives in Biology and Medicine, 35, 220235 .

Thomson, Judith Jarvis 1975 "The Right to Privacy", Philosophy and Public Affairs, 4, 295-314.

Tully, Tim 1991 "Genetic Dissection of Learning and Memory in Drosophila melanogaster", in J. Madden (ed.) Neurobiology of Learning, Emotion, and Affect, New York: Raven Press, 29-66.

Tully, Tim 1994 "Gene disruption of learning and memory: a structure-function conundrum?", Seminars in Neuroscience, (in press). 
Watson, J.D. et.al. 1992 Recombinant DNA (Second Edition) New York: W.H. Freeman.

Wexler, Nancy 1992 "Clairvoyance and Caution: Repercussions from the Human Genome Project", in (Kevles and Hood 1992) 211-243.

Wilson, R. et.al. $1994 \quad " 2.2 \mathrm{Mb}$ of contiguous nucleotide sequence from chromosome III of C. elegans", Nature, 368, 32-38.

Wivel, N. and Walters, L. 1993 "Germ-Line Gene Modification and Disease Prevention: Some Medical and Ethical Perspectives", Science, 262, 533-538. 